SGP-TR-192

\title{
NANOSENSORS AS RESERVOIR ENGINEERING TOOLS TO MAP IN- SITU TEMPERATURE DISTRIBUTIONS IN GEOTHERMAL RESERVOIRS
}

\author{
By \\ Morgan Ames \\ June 2011
}

Financial support was provided through the

Stanford Geothermal Program under

Department of Energy (under contract number DE-FG36-08GO18192).

\section{Stanford University}

Stanford Geothermal Program Interdisciplinary Research in

Engineering and Earth Sciences

STANFORD UNIVERSITY

Stanford, California 



\begin{abstract}
The feasibility of using nanosensors to measure temperature distribution and predict thermal breakthrough in geothermal reservoirs is addressed in this report. Four candidate sensors were identified: melting tin-bismuth alloy nanoparticles, silica nanoparticles with covalently-attached dye, hollow silica nanoparticles with encapsulated dye and impermeable melting shells, and dye-polymer composite time-temperature indicators. Four main challenges associated with the successful implementation of temperature nanosensors were identified: nanoparticle mobility in porous and fractured media, the collection and detection of nanoparticles at the production well, engineering temperature sensing mechanisms that are both detectable and irreversible, and inferring the spatial geolocation of temperature measurements in order to map temperature distribution. Initial experiments were carried out to investigate each of these challenges. It was demonstrated in a slim-tube injection experiment that it is possible to transport silica nanoparticles over large distances through porous media. The feasibility of magnetic collection of nanoparticles from produced fluid was evaluated experimentally, and it was estimated that $3 \%$ of the injected nanoparticles were recovered in a prototype magnetic collection device. An analysis technique was tailored to nanosensors with a dye-release mechanism to estimate temperature measurement geolocation by analyzing the return curve of the released dye. This technique was used in a hypothetical example problem, and good estimates of geolocation were achieved. Tin-bismuth alloy nanoparticles were synthesized using a sonochemical method, and a bench heating experiment was performed using these nanoparticles. Particle growth due to melting was observed, indicating that tin-bismuth nanoparticles have potential as temperature nanosensors.
\end{abstract}





\section{Acknowledgments}

First and foremost, I would like to thank Roland Horne for his expert guidance and support. I would like to thank my research partners Mohammed Al Askar and Chong Liu for their collaboration on this project. Thanks to Steve Connor and Egill Juliusson for their help. I appreciate the weekly brainstorm sessions with Mark McClure, Sarah Pistone, Lilja Magnusdottir, Carla Ko, Kara Bennett, and Kewen Li. Thanks to Brian Anderson for inspiration and encouragement to pursue a career in geothermal energy.

I am grateful to the U.S. Department of Energy for providing funding for this work, under contract number DE-FG36-08GO18192. 



\section{Contents}

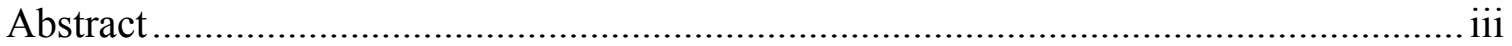

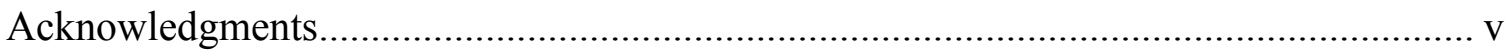

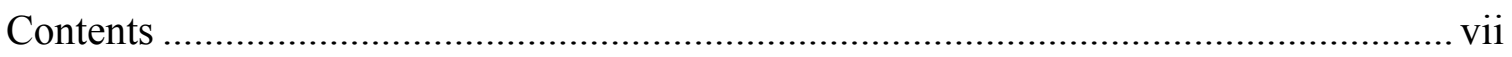

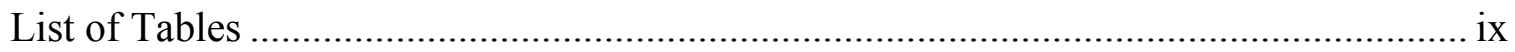

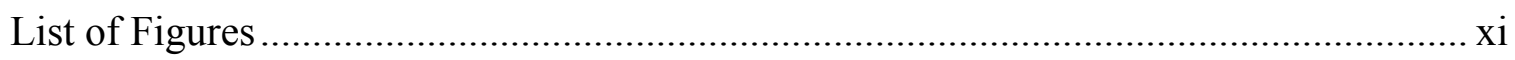

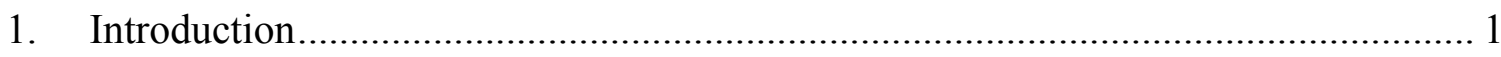

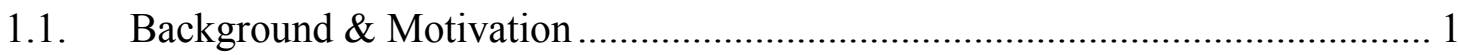

1.1.1. The Role of Geothermal Energy ............................................................... 1

1.1.2. The Importance of Temperature Distribution in Geothermal Reservoirs... 2

1.1.3. Previous Efforts To Measure Reservoir Temperature and Predict Thermal

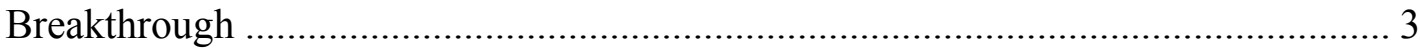

1.1.4. Nanosensors as Tools to Measure Reservoir Temperature.......................... 4

1.2. Objectives and Challenges ....................................................................... 5

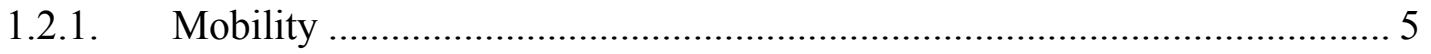

1.2.2. Collection and Detection .................................................................. 7

1.2.3. Irreversible Sensing Mechanism........................................................... 7

1.2.4. Knowing the Geolocation of Temperature Measurement............................. 7

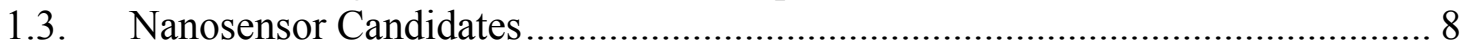

1.3.1. Melting tin-bismuth alloy nanoparticles ............................................... 8

1.3.2. Silica nanoparticles with covalently attached fluorescent dye ................... 8

1.3.3. Hollow silica nanoparticles with encapsulated dye and impermeable

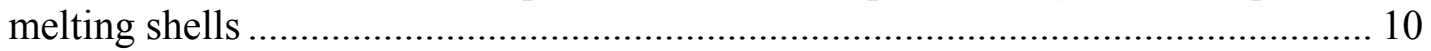

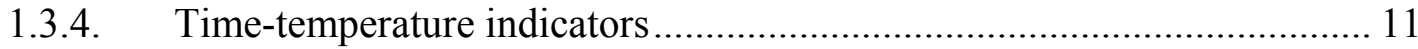

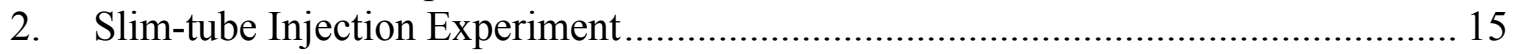

2.1. Experimental Methods ........................................................................... 15

2.1.1. Transducer Calibration ......................................................................... 16

2.1.2. Gas permeability measurement......................................................... 18

2.1.3. Liquid permeability measurement .......................................................... 19

2.1.4. Slim-tube injection experiment............................................................ 21

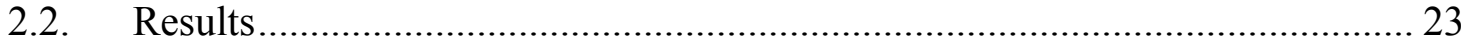

3. Magnetic Collection of Nanoparticles ................................................................ 29

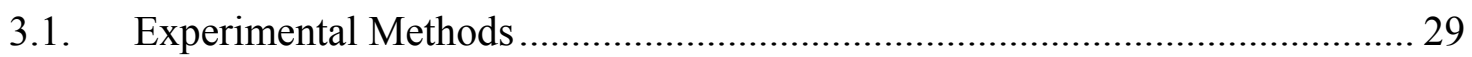

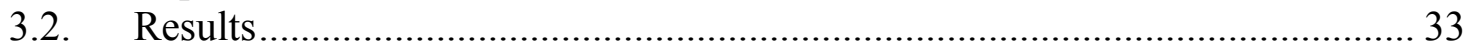

4. Analysis of Tracer Return Curves to Estimate Measurement Geolocation............... 37

4.1. Simple Analytical Model for Return Curve Analysis....................................... 37

4.2. Example Problem...................................................................................... 40 


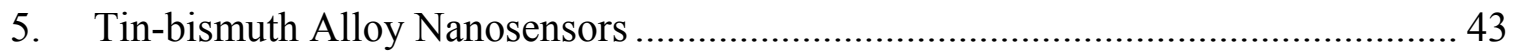

5.1. Synthesis of tin-bismuth alloy nanoparticles ................................................ 44

5.2. Characterization of tin-bismuth alloy nanoparticles ...................................... 44

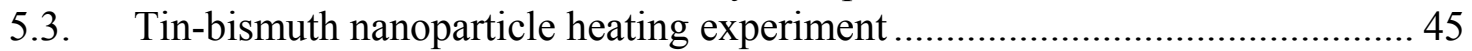

5.4. Tin-Bismuth Nanoparticle Injection Experiments ....................................... 48

6. Conclusions and Future Work ............................................................................ 51

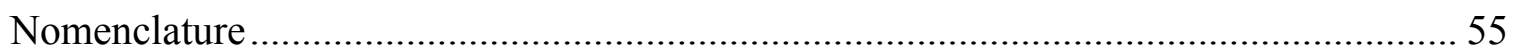

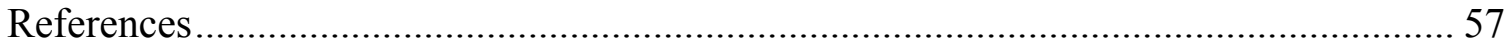




\section{List of Tables}

Table 4-1: Parameter Values Used In Return Curve Analysis Demonstration Problem .. 40

Table 4-2: Estimates of Temperature Measurement Geolocations In Demonstration

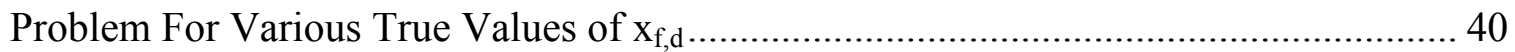





\section{List of Figures}

Figure 1-1: Diagram of particle transport through a rock fracture and the forces that govern it. The inset shows forces that are important when particles are close to rock surfaces. Reproduced from Reimus (1995).

Figure 1-2: SEM images showing tin-bismuth nanoparticles before and after heating to $210^{\circ} \mathrm{C}$ 8

Figure 1-3: Emission spectra of dye-attached silica nanoparticles before and after heating to $200^{\circ} \mathrm{C}$. Reproduced from Alaskar et al. (2011). 9

Figure 1-4: Cartoon of dye-release scheme triggered by the melting of an impermeable shell. 10

Figure 1-5: (a) Photoluminescence (PL) emission spectral shift that occurs upon heating of TTIs and (b) the ratio of dispersed to aggregated emission intensity as a function of heating time with a regression curve. Reproduced from Sing et al. (2009). 12 Figure 2-1: Photographs of (a) the $25 \mathrm{~m}$ stainless steel slim-tube and (b) the $10 \mathrm{~m}$ polypropylene slim tube 16

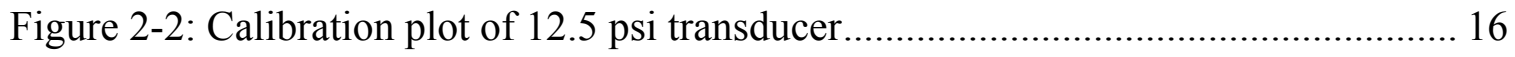

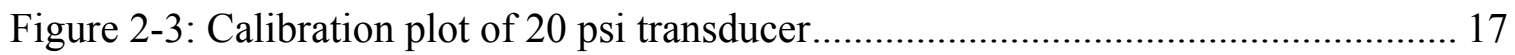

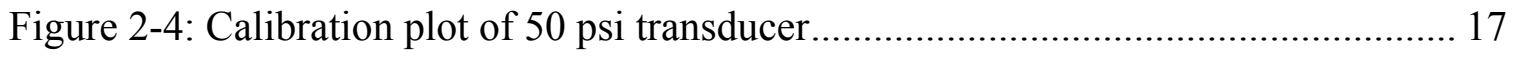

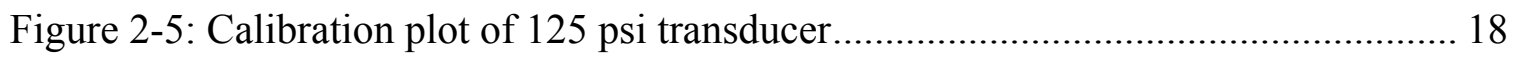

Figure 2-6: Schematic of the experimental apparatus used for the measurement of the gas

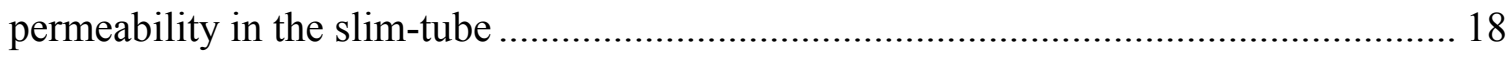

Figure 2-7: Gas permeability versus the reciprocal of mean pressure........................... 19

Figure 2-8: Schematic of the experimental apparatus used for the measurement of the

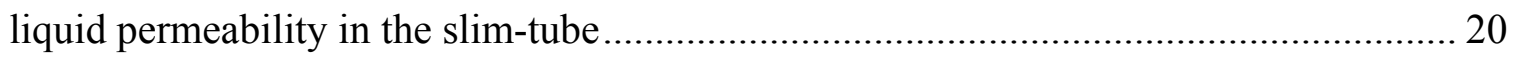

Figure 2-9: Schematic of the experimental apparatus used for the nanoparticle injection experiment in the slim-tube. 
Figure 2-10: Photograph of apparatus used for the nanoparticle injection experiment in

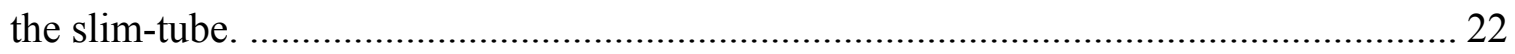

Figure 2-11: Size distribution of influent silica nanoparticles as measured by DLS. ..... 22 Figure 2-12: SEM image showing a sample of influent silica nanoparticles used in injection. 23

Figure 2-13: Photograph of effluent samples in order of collection. Note that the cloudy or opaque samples are more concentrated with silica nanoparticles. 24 Figure 2-14: SEM images of silica nanoparticles in effluent samples. Note that these images correspond to the samples shown in Figure 2-13 with the same labels. 24 Figure 2-15: DLS results of effluent samples. Intensity at $350 \mathrm{~nm}$ diameter is shown, indicating that detectable amounts of nanoparticles were present in the effluent from 0.5 to 1.6 pore volumes. 25

Figure 2-16: Permeability measurements taken during the silica nanoparticle injection and backflushing experiments.

Figure 2-17: SEM images of effluent samples taken from the (a) $3^{\text {rd }}$ and (b) $8^{\text {th }}$ injected pore volumes. 26

Figure 2-18: SEM image of effluent sample taken from the $1^{\text {st }}$ pore volume of the backflushing experiment. 26

Figure 3-1: Schematic of experimental apparatus used in the magnetic collection experiment.. 30

Figure 3-2: Magnetic field of neodymium block magnets. Reproduced from K\&J Magnetics. 30

Figure 3-3: Magnetic pull force between two neodymium magnets as a function of distance. The point on the curve corresponds to $13.31 \mathrm{lb}_{\mathrm{f}}$ at a distance of $0.125 \mathrm{in}$., or the radius of the collection tube used. Reproduced from K\&J Magnetics. 31 Figure 3-4: SEM image of iron oxide nanoparticles coated with silica. 32 Figure 3-5: Photograph of trapped nanoparticles after the removal of the magnetic trap.33 Figure 3-6: Visual comparison of trapped nanofluid sample and 142.5 to 1 dilution of original nanofluid. 34 Figure 3-7: Absorbance spectra of suspensions of iron oxide nanoparticles coated with silica. 35 
Figure 3-8: Correlation of concentration to absorbance for dilutions of iron oxide nanofluid with known concentrations. 35

Figure 4-1: Cartoon of temperature distribution in a geothermal reservoir with a thermal front at position $\mathrm{x}_{\mathrm{f}}$ 38

Figure 4-2: Return curve data and fits for A) $\left.\left.x_{f}=50 \mathrm{~m}, \mathrm{~B}\right) \mathrm{x}_{\mathrm{f}}=350 \mathrm{~m}, \mathrm{C}\right) \mathrm{x}_{\mathrm{f}}=650 \mathrm{~m}$, and D) $x_{\mathrm{f}}=950 \mathrm{~m}$. Note that released dye experiences breakthrough first because it is carried a distance $\mathrm{x}_{\mathrm{f}}$ by the nanosensor, which has a retardation factor of 1 , while the conservative tracer has a retardation factor of 2 .

Figure 4-3: Objective function surface for fitting the return curve of the reactive tracer when $\mathrm{x}_{\mathrm{f}}=50 \mathrm{~m}$.

Figure 4-4: Objective function surface for fitting the return curve of the reactive tracer when $\mathrm{x}_{\mathrm{f}}=50 \mathrm{~m}$, zoomed in near the minimum of $\left(\mathrm{V}_{\mathrm{x}, \mathrm{n}}=1000 \mathrm{~m}^{3}, \mathrm{~V}_{\alpha, \mathrm{n}}=500 \mathrm{~m}^{3}\right)$. Note that the point chosen by the solver was $\left(\mathrm{V}_{\mathrm{x}, \mathrm{n}}=268.3 \mathrm{~m}^{3}, \mathrm{~V}_{\alpha, \mathrm{n}}=180.8 \mathrm{~m}^{3}\right) \ldots \ldots \ldots \ldots \ldots \ldots . . . . .42$ Figure 5-1: Phase diagram of tin-bismuth. Reproduced from National Institute of Standards and Technology. 43

Figure 5-2: Logarithmic particle size distribution based on hydrodynamic diameter for original tin-bismuth nanoparticle sample. 44

Figure 5-3: SEM images of tin-bismuth nanoparticles at higher magnification. 45

Figure 5-4: Experimental apparatus for tin-bismuth heating experiment 46

Figure 5-5: Logarithmic particle size distribution based on hydrodynamic diameter for heated tin-bismuth nanoparticle sample. 46

Figure 5-6: Comparison of logarithmic particle size distribution based on hydrodynamic diameter for original and heated tin-bismuth nanoparticle samples. 47

Figure 5-7: SEM images showing heated tin-bismuth nanoparticles. 47 Figure 5-8: Permeability measurements during injection of tin-bismuth nanoparticles into Berea sandstone core. Reproduced from Alaskar et al. (2011). .................................. 48 Figure 5-9: SEM images showing tin-bismuth nanoparticles in the effluent from (a) injection experiment and (b) backflushing experiment. Reproduced from Alaskar et al. (2011). 49

Figure 5-10: SEM images of the inlet to the Berea sandstone core with evidence of nanoparticle trapping. Reproduced from Alaskar et al. (2011). 49 
Figure 5-11: Visual observation of tin-bismuth nanoparticles in the effluent samples from the slim-tube injection experiment. Reproduced from Alaskar et al. (2011). .................. 50 Figure 5-12: SEM images showing tin-bismuth nanoparticles of all sizes in the effluent from the slim-tube injection experiment. Reproduced from Alaskar et al. (2011)........... 50 


\section{Chapter 1}

\section{Introduction}

\subsection{Background \& Motivation}

\subsubsection{The Role of Geothermal Energy}

With global populations on the rise and the increasing threat associated with climate change, the need for the development of low emission energy resources is clear. Geothermal energy, which originates from the underground heat of the earth (Sankaran, 2002), has the advantage of being a low-emission, baseload energy resource. Unlike other alternative energy resources, geothermal energy production does not fluctuate with time of day or season. Furthermore, geothermal energy is an indigenous resource that cannot be exported easily and thus delivers energy security and jobs near its deployment. Humankind has been using geothermal energy for millennia for balneological purposes and as a resource to generate electricity for over a century.

The vast majority of geothermal energy used by humans today comes from high grade hydrothermal systems, which meet all three requirements necessary for economic extraction of geothermal energy: heat, fluid in place to carry the heat to the surface, and rock permeability that allows the fluid to flow. Due to their anomalous nature, hydrothermal systems are limited in both geographic range and overall potential to play a significant role in energy use. In 2010, approximately 67,246 GWh of geothermal electricity were generated worldwide from an installed capacity of $10.7 \mathrm{GW}_{\mathrm{e}}$ (Bertani, 2010). In the same year, an additional $121,696 \mathrm{GWh}$ of heat were generated for direct use applications from an installed capacity of $50.6 \mathrm{GW}_{\text {th }}$ (Lund et al., 2010). To put these numbers in perspective, consider that 7.9 million GWh of coal-fired electricity were generated worldwide in 2007 (U.S. Energy Information Administration).

Despite its somewhat limited use, geothermal energy is an abundant resource. Hermann (2006) estimated the geothermal exergy flow from the mantle into the crust to be $32 \mathrm{TW}$.. Hermann (2006) also estimated the total resource size to be of $2 \times 10^{19} \mathrm{TJ}$ and the replenishment time scale to be on the order of days to years. Enhanced Geothermal Systems (EGS) are geothermal systems in which permeability is created artificially by means of hydraulic fracturing, and water (or perhaps $\mathrm{CO}_{2}$ ) is circulated as a working fluid. Because heat is the only requirement needed to be provided by nature in this concept, EGS implementation has the potential to greatly expand geothermal energy capacity and the geographic range of its use. A 2006 study projected that EGS development in the United States could yield $100 \mathrm{GWe}$ of installed capacity within 50 years (Tester et al., 2006). Furthermore, it was estimated in the same study that 200,000 EJ (the equivalent of 2000 times the primary energy consumption by the United States in 
2005) could eventually be extracted using EGS in the United States alone (Tester et al., 2006). Nonetheless, there are a number of key challenges to be faced in EGS development before this goal can be realized. In the reservoir creation stage, the main challenges are creating a fracture surface that is large enough to enable sufficient production and extensive enough to avoid short circuiting, creating fracture networks that are capable of accepting economical flowrates, and minimizing induced microseismicity associated with the fracturing process (Horne 2011). In the reservoir production stage, the main challenges are obtaining information regarding the geometry of conductive fractures as well as the temperature distribution around these fractures. The latter category of challenges also applies to hydrothermal reservoirs.

\subsubsection{The Importance of Temperature Distribution in Geothermal Reservoirs}

Knowledge of in-situ temperature distribution is essential to being able to evaluate the economics and sustainability of geothermal energy extraction. In the most fundamental sense, the energy in place is a direct function of the temperature distribution. Having this information at the beginning of a project enables one to size the power station appropriately. Furthermore, knowing the temperature distribution once a reservoir has been cooled by years of reinjection would allow reservoir engineers to predict reservoir life more accurately by comparing measurements to the initial temperature distribution.

Temperature decline due to the cooling of rock near the fluid-rock interface is a significant problem in geothermal reservoirs where reinjection is practiced. Reinjection of fluids is an inherent part of the EGS concept because by definition, these reservoirs do not have fluid in place. Reinjection is also practiced in most conventional hydrothermal reservoirs. The synergistic purposes of reinjection are to maintain reservoir pressure, dispose of wastewater, and to increase the thermal sweep efficiency (Horne 2010). In different experiences in the past, geothermal reinjection has caused both productivity enhancement and damage (Horne 2010). As a result of injection of cold fluid, the heat in reservoir rock is depleted in the vicinity of fluid-rock interface. This depletion spreads from the injection well in what is referred to as the cooling front. After the thermal front arrives at the production well, thermal breakthrough is said to have occurred (Horne 2010). Thermal breakthrough is of utmost importance in reservoirs where reinjection is performed, because both the enthalpy and flowrate of the produced fluid decreases (Horne 2010). The flowrate decreases because the decrease in enthalpy is accompanied by an increase in the density of the two-phase fluid, making it more difficult to lift it out of the wellbore (Horne 2010). This exact problem occurred in well H-4 at Hatchobaru geothermal field in Japan and led to the well being abandoned (Horne 2010).

Tools capable of mapping the temperature distribution in geothermal reservoirs would be useful at various stages of project life. Knowing reservoir temperature distribution at the beginning of extraction would facilitate more optimal production strategies and reduce costs and associated risks. For example, it is common in geothermal reservoir models to assume a reservoir consists of isothermal layers. Better resolution with respect to temperature would allow more physically accurate models to be constructed, which would make geothermal reservoir simulators more powerful forecasting tools. 
Additionally, knowing the temperature distribution after the cooling front has advanced would facilitate decisions to adjust production strategy in order to increase profit or preserve a resource.

\subsubsection{Previous Efforts To Measure Reservoir Temperature and Predict Thermal Breakthrough}

Horne (2010) described accurate forecasting of thermal breakthrough as one of the main goals of evaluating a reinjection scheme. To this end, he derived an analytical solution for a linear flow path which expresses the thermal breakthrough time $t_{t h}$ as a function of the chemical breakthrough time $t_{c}$ and the fracture aperture $b$ :

$$
t_{t h}=t_{c}+4.234\left(\frac{t_{c}}{b}\right)^{2} \frac{K_{r} \rho_{r} C_{r}}{\rho_{w} C_{w}}
$$

where $K_{r}$ is the thermal conductivity of the rock, $\rho_{w}$ and $\rho_{r}$ are the respective densities of water and rock, and $C_{w}$ and $C_{r}$ are the respective heat capacities of water and rock. While this estimate is based on a simplified linear flow path and is not accurate in all cases, it is probably the best estimate that can be made during the initial phases of a project. Shook (1999) also developed a method to predict thermal breakthrough for single-phase flow in a geothermal reservoir from tracer return curves and verified the method by matching his predictions to simulation results. This method neglected dispersion and thermal conductivity so that the ratio of thermal velocity to fluid velocity could be taken as constant and employed empirical transformations of both concentration and temperature.

Another way to predict thermal breakthrough is to measure the temperature distribution in the reservoir at different stages of reservoir life. This method would provide real-time information about the location of the thermal front and has the potential to be a powerful forecasting tool. However, current technology only allows for temperature measurement at or near wellbores. Over the past few decades, a great deal of effort has been devoted to the problem of mapping temperature distribution further into the formation, but this has not been demonstrated successfully in practice. Numerous papers in the literature suggest the use of reactive tracers to invert for formation temperature based on Arrhenius reaction kinetics. Robinson et al. (1984) suggested that reactions with temperaturedependent rates can be engineered within a geothermal reservoir to determine its temperature distribution as a function of residence time. Tester et al. (1986) proposed the use of a reactive tracer to map the progress of thermal fronts in particular flowpaths in EGS. Tester et al. (1987) established criteria for selection of reactive tracers, indicating that compounds with Arrhenius parameters that yield characteristic reaction times on the order of magnitude of the mean fluid residence time could be used to measure reservoir temperature. Robinson and Birdsell (1987) proposed that the hydrolysis of bromobenzene derivatives could potentially be used to the same end in the temperature range of 150$275^{\circ} \mathrm{C}$, and emphasized the need for a field demonstration of this concept at Fenton Hill. Rose and Adams (1994) performed a field study in which a conservative tracer (fluorescein) and a reactive tracer (rhodamine WT) were injected into the Steamboat Hills reservoir. The decay kinetics of rhodamine WT were extrapolated to estimate an effective reservoir temperature of $163^{\circ} \mathrm{C}$. However, the spatial temperature distribution 
was not measured, and the authors stated that rhodamine WT could only be used for this type of measurement in a limited temperature range.

More recently, Behrens et al. (2009) showed in simulations that when a reservoir has been cooled but not to the point of thermal breakthrough, the reactive solute tracer method is not sufficiently sensitive to predict thermal breakthrough. These authors also noted that currently there are no compounds with the desired Arrhenius parameters for temperature measurement, and that kinetics measured under laboratory conditions might not hold at reservoir conditions. Plummer et al. (2010) suggested that the sensitivity of reactive tracers could be improved by performing push-pull tests or reaction quenching in a flow-through test. Nottebohm et al. (2010) also discussed the use of reactive tracers in push-pull tracer experiments. Plummer et al. (2011) suggested that a reactive tracer that experiences a sequence of reactions might be sensitive enough to provide information about thermal breakthrough.

\subsubsection{Nanosensors as Tools to Measure Reservoir Temperature}

The focus of this research is a variation of the reactive tracer concept, in which nanoparticle tracers are used as flow-through sensors that undergo detectable and irreversible changes at a particular threshold temperature. Nanoparticles have excellent potential as temperature measurement tools because they can be synthesized with a great degree of control over their structure and both physical and chemical properties, making possible a host of different sensing mechanisms. Furthermore, their small size enables them to pass through pore spaces.

In recent years, nanoparticles have received significant attention for purposes analogous to those of this project. Several authors have proposed the use of smart nanoparticle sensors to infer petroleum reservoir properties in situ. Saggaf (2008) envisioned a future where "nanorobots" capable of measuring temperature, pressure, and fluid type and storing these measurements in on-board memory would be used. Kanj et al. (2009), Alaskar et al. (2010), and Yu et al. (2010) all performed initial coreflooding experiments to investigate the transport of nanoparticles in porous media. Reimus (1999) performed field experiments in which colloids were injected into fractured granite in order to study their transport properties for groundwater contamination applications. Reimus (1995) defined a "colloid" as a particle that falls within the size range of $1 \mathrm{~nm}$ to about $1 \mu \mathrm{m}$, which is approximately the size range investigated in this project. Rose et al. (2011) suggested the use of surface-modified quantum dots to measure the fracture surface area between two wells in EGS applications. Redden et al. (2010) proposed the use of contained nanoreactors that make use of thermoluminscence or polymer racemization to infer the thermal history of a geothermal reservoir. Nanoparticles have also been used for temperature sensitive in vivo drug release in biomedical applications (Sutton et al., 2007). While it is not trivial to extend this concept from the biological temperature regime to the much higher geothermal temperature regime, this scheme shows promise nonetheless. 


\subsection{Objectives and Challenges}

The overall goal of this project was to develop functional nanosensors capable of mapping the temperature and pressure distributions in geothermal reservoirs. Measuring temperature was the primary goal, because temperature is of greater significance in geothermal applications. This concept involves a number of technical challenges that must be overcome for the project to be successful. This report includes discussion of preliminary work toward overcoming each of these challenges.

\subsubsection{Mobility}

For temperature nanosensors to be implemented successfully, they must be transported through fractured reservoir rock without much filtration and without reducing reservoir permeability. This necessitates that the nanoparticles remain in suspension (i.e. the particles are hydrophilic and do not settle due to gravitational forces), experience little or no aggregation, and do not experience appreciable adsorption to rock surfaces (Kanj et al., 2009). Reimus (1995) performed an extensive study of colloidal transport in fractures and concluded that if the particles do not adsorb to rock surfaces, they actually tend to move more rapidly than the average fluid velocity and experience breakthrough sooner than solute tracers. Reimus attributed this to colloids being too large to experience matrix diffusion and having a tendency to stay in fluid streamlines. Reimus suggested that due to this behavior, colloids might be useful in tandem with conventional solute tracers to actively measure the effect of matrix diffusion. Reimus described fluid advection, gravitational settling, and, to a lesser extent, diffusion, to be the forces that govern colloid transport in fractures. He also suggested van der Waals forces and electrostatic forces can also impact transport, but only when particles are very close to rock surfaces. These forces are illustrated in Figure 1-1, which is reproduced from Reimus (1995). 


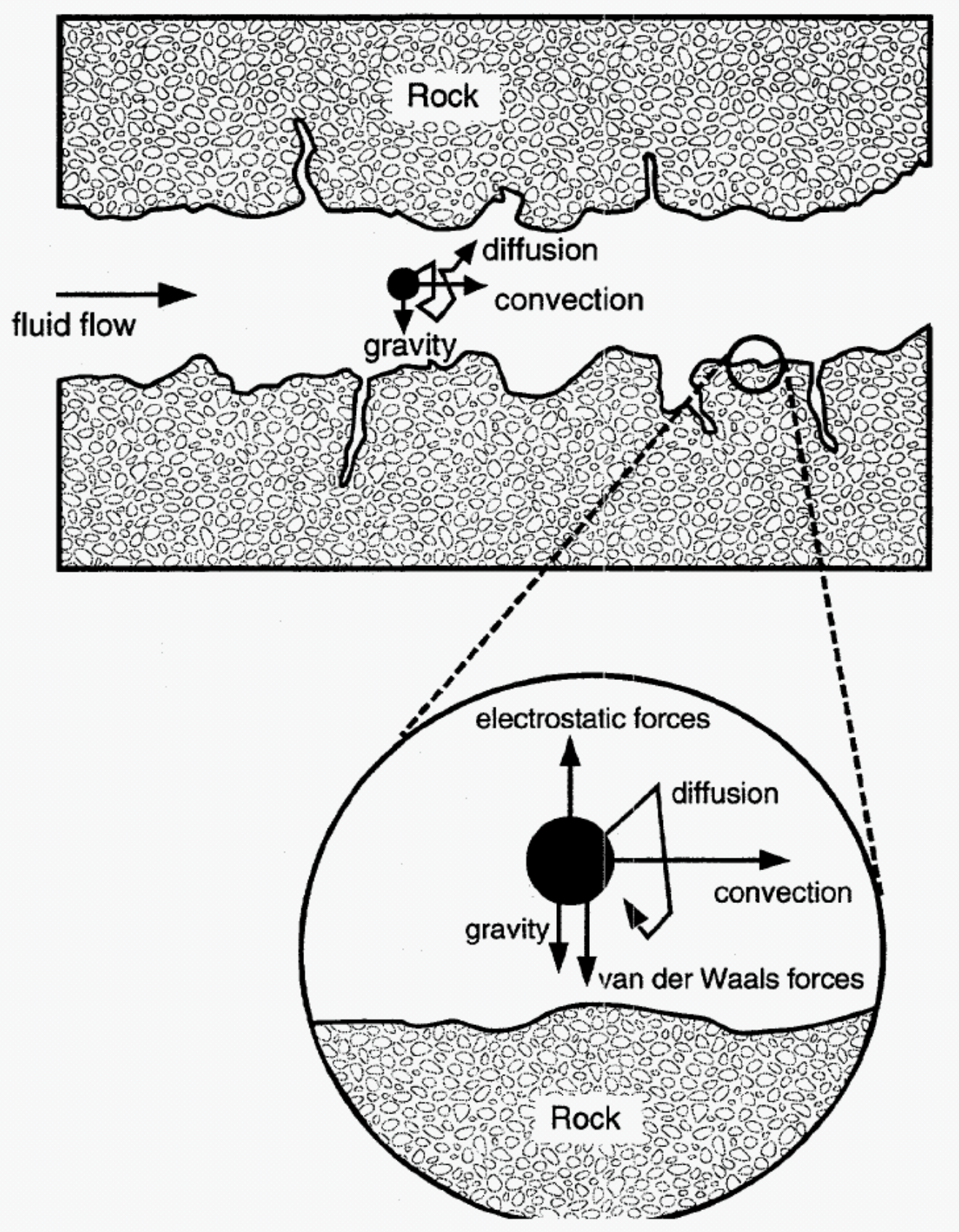

Figure 1-1: Diagram of particle transport through a rock fracture and the forces that govern it. The inset shows forces that are important when particles are close to rock surfaces. Reproduced from Reimus (1995).

It is clear that particle mobility is a very complex problem that has a very large impact on the success of nanosensors. The nanoparticles used must be small enough to fit through the fractures and pore spaces while experiencing no gravitational settling, but they must also be large enough so as not to diffuse or aggregate, which could lead to particle filtration (Cumbie and McKay, 1999). The surface chemistry of the nanoparticles should be such that the particles tend to stay suspended in the geothermal fluid and do not adsorb 
physically or chemically to the rock surfaces. On the other hand, it may be possible to exploit the differences in transport between nanoparticles and solute tracers to provide important information about temperature measurements, which is discussed in more detail in Chapter 4 of this report. An experimental investigation of nanoparticle mobility is described in Chapter 2.

\subsubsection{Collection and Detection}

For the nanosensors to provide information about the reservoir, they must be collected from the produced fluid, and they must be detectable at low concentrations because reservoir volumes are orders of magnitude larger than those of injected tracers. It is also desirable to be able to measure the concentration of these nanoparticles in order to construct return curves. The use of magnetic nanoparticles may enable their collection from produced fluid using powerful magnets. A preliminary experimental investigation of magnetic collection of nanoparticles is discussed in Chapter 3 of this report. Nanomaterials can have unique and detectable optical or fluorescent properties in dilute suspensions, and this may also enable measurement of nanoparticle concentration. Furthermore, sensing mechanisms that involve changes in these detectable properties may provide convenient means of measurement.

\subsubsection{Irreversible Sensing Mechanism}

The flow-through nanosensor concept inherently involves some irreversible change that can be accurately correlated to temperature. Exploiting a reversible change would necessitate the need for on-board memory that could be interrogated after collection. While sensors with such memory would be powerful, they are not considered in the short term scope of this work. Keeping this in mind, it is desirable to make use of a sensing mechanism that provides as much information about the temperature distribution as possible without interfering with other sensor requirements. For example, a common sensing mechanism for controlled drug release makes use of a temperature-triggered switch from hydrophilic to hydrophobic behavior (Sutton et al., 2007). However, as this would likely have a negative impact on particle mobility, it is not a promising sensing mechanism for application in geothermal reservoirs.

There are many conceivable sensing mechanisms for temperature. Mechanisms considered thus far include size or shape change due to melting, the release of an attached dye after a thermosensitive bond is cleaved, the release of an encapsulated dye after an impermeable shell melts, and permanent fluorescence change due to dye aggregation or dispersion in a polymeric nanoparticle. An investigation of the first sensing mechanism was performed in this study and is discussed in Chapter 5 .

\subsubsection{Knowing the Geolocation of Temperature Measurement}

While nanosensors that undergo a detectable, irreversible change at a threshold temperature of interest have the potential to tell us whether this threshold was encountered in a geothermal reservoir, this is only part of the requirement. In order to map the temperature distribution, we must also know the geolocation of the temperature measurement. Certain sensing mechanisms, such as dye release, show potential for the 
development of techniques to analyze tracer returns to estimate measurement geolocations. A preliminary study was performed to demonstrate the potential of dyereleasing nanosensors to infer geolocations from return curves and is discussed in Chapter 4 . This capability will render some sensing mechanisms more useful than others.

\subsection{Nanosensor Candidates}

At this stage, four promising nanosensor candidates with various sensing mechanisms have been identified and chosen for investigation: melting tin-bismuth alloy nanoparticles, dye-attached silica nanoparticles, hollow silica nanoparticles with encapsulated dye and impermeable melting shells, and dye-polymer composite timetemperature indicators. It makes sense for the sensing mechanism to be the starting point of candidate nanosensor selection, as it defines the function of the nanosensor and dictates the properties required. The ideal sensor will have good mobility in reservoir rock, predictable sensitivity to temperature, recovery and detection capabilities, capability to infer measurement geolocation, relatively low in cost, and environmentally benign. Each candidate sensor will be evaluated according to these criteria.

\subsubsection{Melting tin-bismuth alloy nanoparticles}

One of the simplest conceivable sensing mechanisms is a change in particle size or shape caused by melting. Tin-bismuth alloy nanoparticles were identified as appropriate candidates to demonstrate size change due to melting, because their melting point can be tuned within a wide temperature range that is appropriate for geothermal applications. Tin-bismuth nanoparticles were synthesized, and experimental investigations were performed to evaluate their temperature sensitivity and mobility in porous media. This sensing mechanism was observed clearly using Scanning Electron Microscopy (SEM), as shown in Figure 1-2. More details are provided in Chapter 5 of this report.
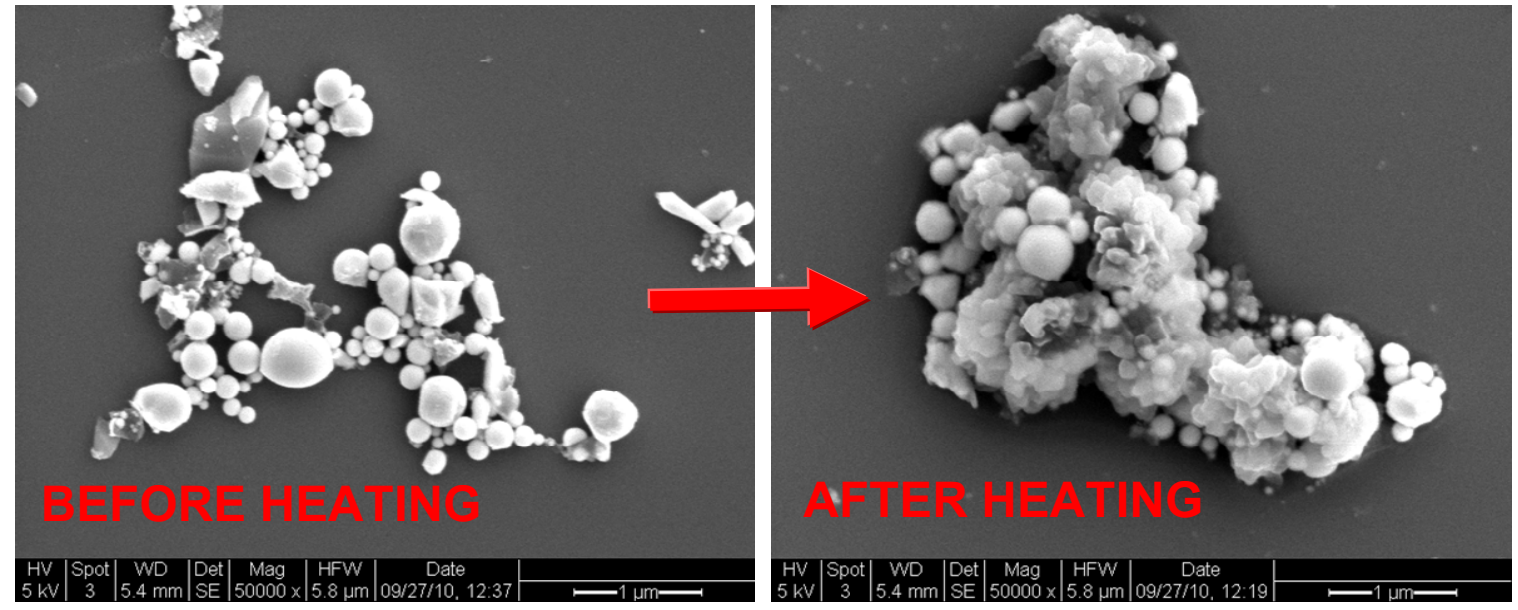

Figure 1-2: SEM images showing tin-bismuth nanoparticles before and after heating to $210^{\circ} \mathrm{C}$

\subsubsection{Silica nanoparticles with covalently attached fluorescent dye}

As it is common practice to use fluorescent dyes as tracers in geothermal reservoirs, a dye-release temperature-sensing scheme would be a convenient means of measuring 
temperature. Additionally, if a thermally stable dye with sufficiently low detection limits were employed, this sensing scheme would eliminate the need for nanoparticle collection at the production well, which is a significant technical challenge itself. Finally, the dyerelease sensing scheme may enable the estimation of measurement geolocation, as discussed in Chapter 4.

$\mathrm{Wu}$ et al. (2008) synthesized sensors in which fluorescent dye was attached to the surfaces of porous silicon microparticles, resulting in a different emission spectrum from that of the free dye due to energy transfer. Inspired by this, a similar sensing mechanism was devised by colleague Chong Liu, in which a thermosensitive bond breaks upon exposure to high temperature, leading to dye release, which is detectable by the unique emission spectrum of the free dye (Alaskar et al., 2011). As reported by Alaskar et al. (2011), Oregon 488 dye was covalently linked to silica nanoparticles. After these particles were heated on a substrate, a pronounced change in their emission spectra was observed, as shown in Figure 1-3.

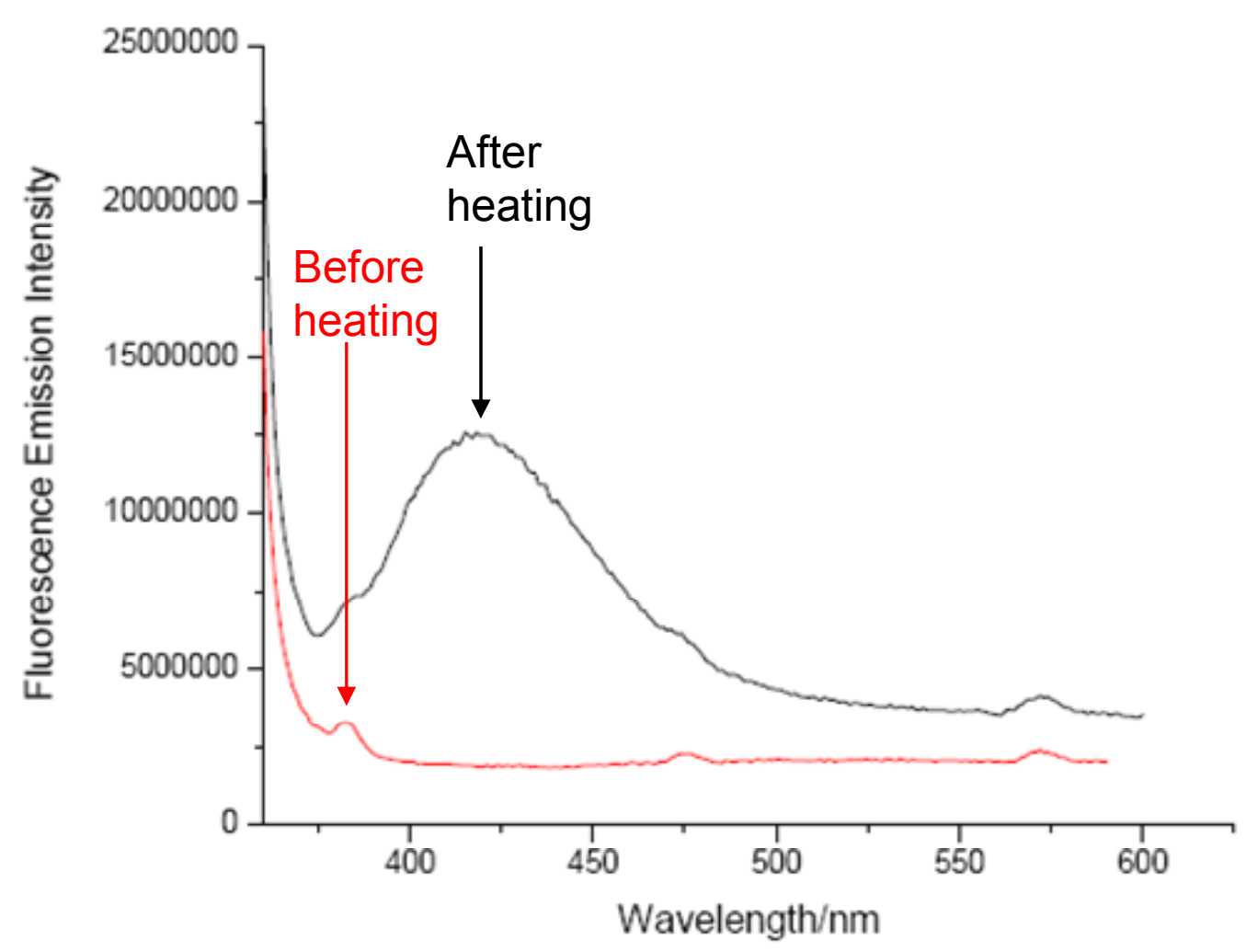

Figure 1-3: Emission spectra of dye-attached silica nanoparticles before and after heating to $200^{\circ} \mathrm{C}$. Reproduced from Alaskar et al. (2011).

These nanosensors have been demonstrated to show potential for geothermal temperature measurement. More extensive evaluations of their temperature sensitivity and their mobility in porous and fractured media are planned. 


\subsubsection{Hollow silica nanoparticles with encapsulated dye and impermeable melting shells}

Another dye release mechanism is the release of encapsulated dye from a hollow porous silica nanoparticle after an impermeable shell melts at the sensing temperature. This candidate has the advantages of convenient measurement, possible elimination of the need to collect the nanosensors from the produced fluid, and possible capability to infer measurement geolocation. Botterhuis et al. (2006) have synthesized hollow silica spheres with encapsulated dye and demonstrated controlled-release behavior in aqueous media. The dye release was found to exhibit two types of behavior: rapid release of dye immobilized in the meso- and macropores via diffusion, and slow, steady release of dye incorporated into the silica walls after the walls dissolved around it.

If these hollow silica nanoparticles were coated with a material impermeable to dye diffusion and with an appropriate melting point, temperature-sensitive dye release could be achieved for geothermal applications, as illustrated in Figure 1-4. Possible candidates for the coating material include tin-bismuth and polymers with melting points in the temperature range of geothermal interest. Technical challenges anticipated include the development of a suitable coating process, measurement precision, and particle mobility in reservoir rock.

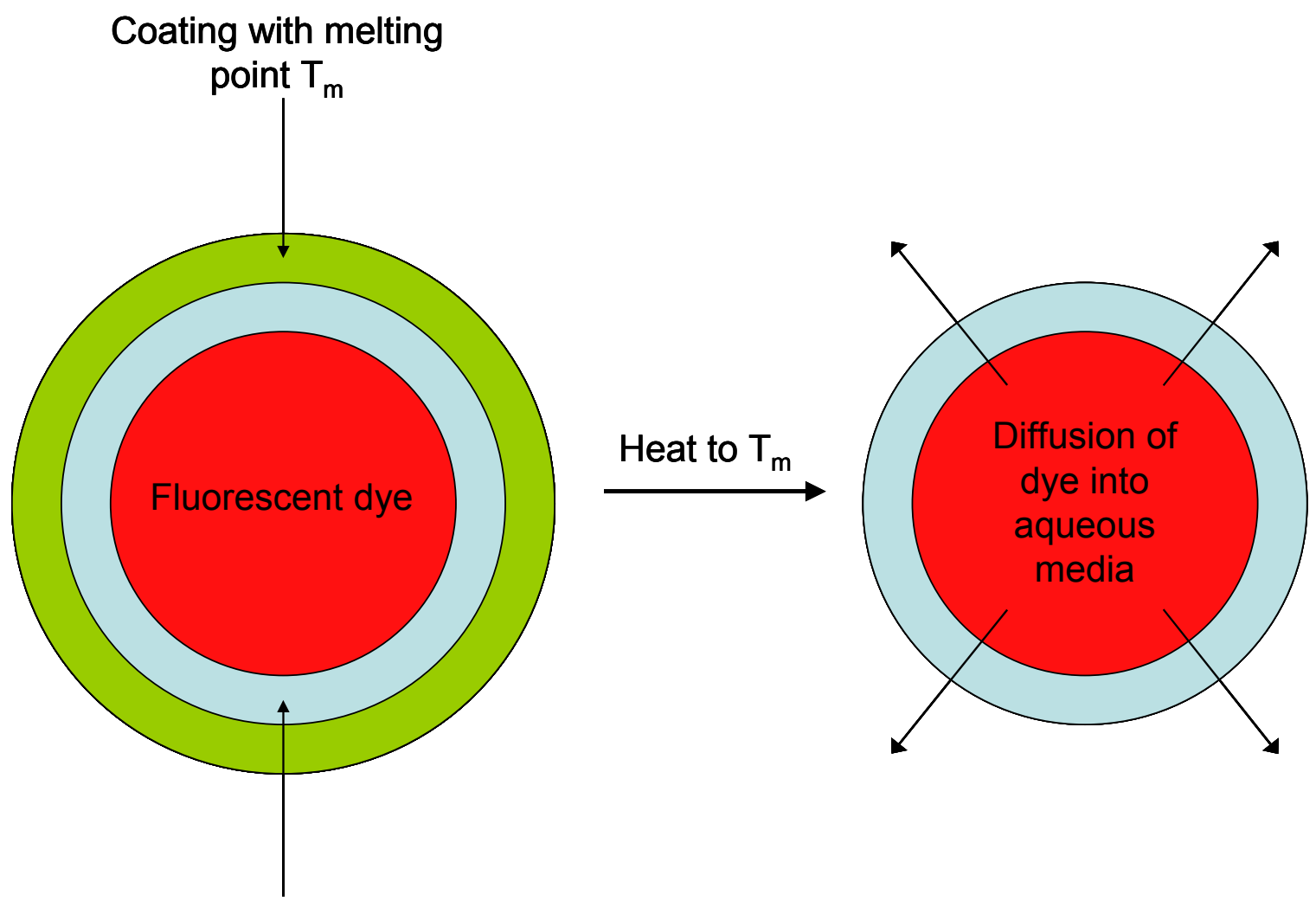

Hollow silica sphere

Figure 1-4: Cartoon of dye-release scheme triggered by the melting of an impermeable shell. 


\subsubsection{Time-temperature indicators}

Sing et al. (2009) have developed time-temperature indicators (TTIs) that have been demonstrated to work in high temperature regimes $\left(130^{\circ} \mathrm{C}-200^{\circ} \mathrm{C}\right)$. As most temperature sensors in the literature operate in lower temperature ranges found in biomedical applications, this type of sensor is a very promising candidate. The TTIs developed by Sing et al. were films of dye/polymer blends that underwent irreversible fluorescence changes when the dye aggregated after being heated above the glass transition temperature $T_{g}$ of the polymer.

In the work of Sing et al. (2009), the dyes 4,4'-bis(2-benzoxazolyl)stilbene or cyanosubstituted oligo( $p$-phenylene vinylene) were kinetically trapped in thermodynamically unstable dispersed states within ethylene/norbornene copolymers by quenching the blend below $T_{g}$ during synthesis. Heating the TTIs above $T_{g}$ caused irreversible phase separation to occur by means of dye aggregation. This aggregation allowed excimers to form and charge-transfer interactions to occur, resulting in permanent changes in fluorescence, as shown in Figure 1-5. 

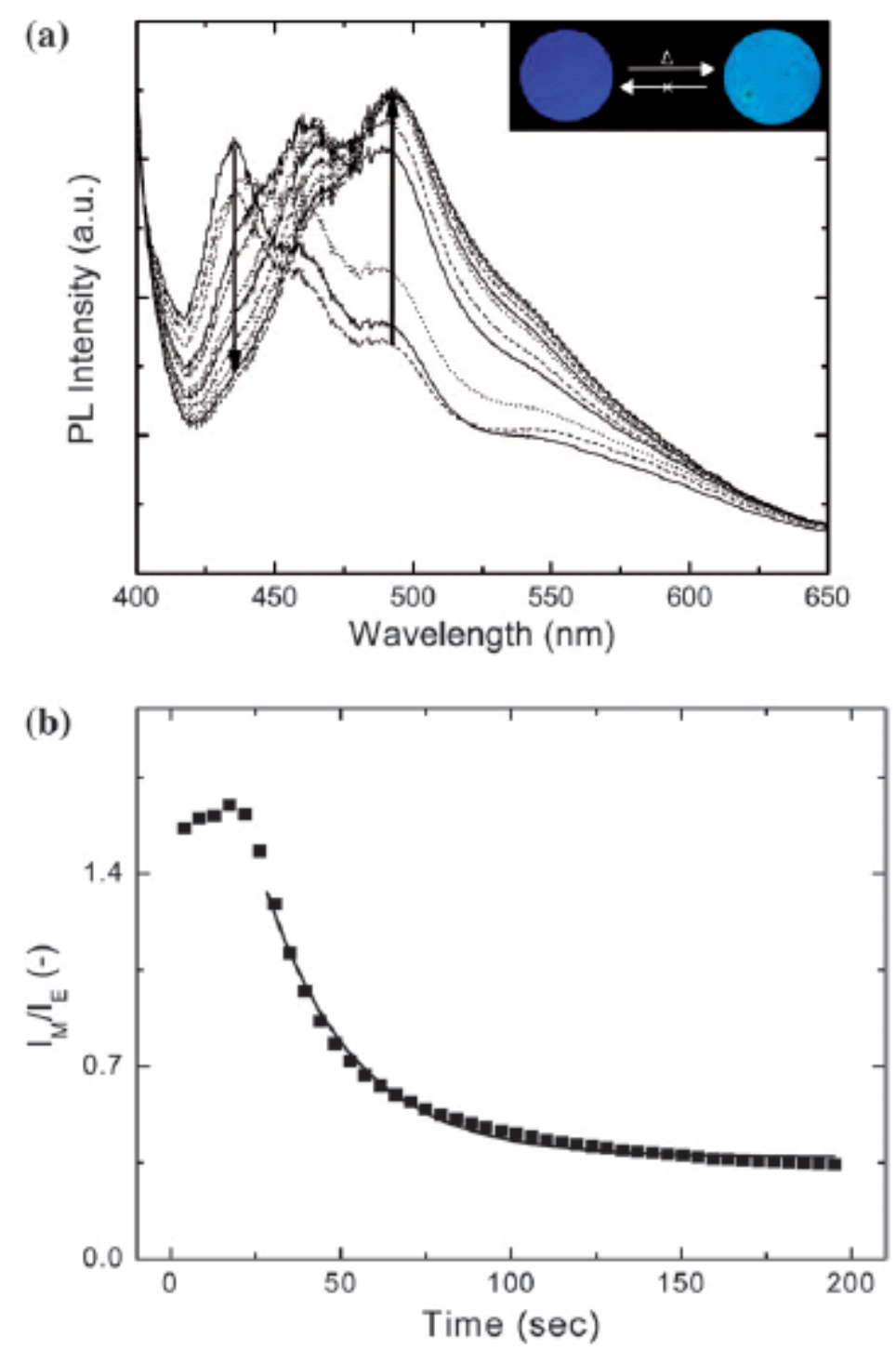

Figure 1-5: (a) Photoluminescence (PL) emission spectral shift that occurs upon heating of TTIs and (b) the ratio of dispersed to aggregated emission intensity as a function of heating time with a regression curve. Reproduced from Sing et al. (2009).

The kinetics of dye aggregation upon heating exhibited predictable behavior, with the kinetic rate constant following Arrhenius-type temperature dependence. Moreover, the time scale of aggregation can be tuned between seconds and days by changing the dye concentration, choice of dye, or choice of host polymer. Thus, TTIs show potential for providing information about the time of exposure to a given temperature, which could be inverted to estimate the location of the thermal front within a geothermal reservoir. Three main technical challenges are anticipated regarding the development of TTIs for geothermal applications. The first is the synthesis of nano- or microparticles of the dyepolymer blends with the same capabilities, as the sensors described by Sing et al. (2009) are films, not particles. The second challenge is the precision with which such a sensor can measure reservoir temperature. The final challenge is mobility of the particles through reservoir rock. 



\section{Chapter 2}

\section{Slim-tube Injection Experiment}

The success of the geothermal nanosensor concept is contingent upon the ability to transport sufficient quantities of nanoparticles from the injection well to the production well without changing the reservoir permeability. An injection experiment in a $10 \mathrm{~m}$ slim-tube packed with sand was carried out to investigate the mobility of nanoparticles in porous media over length scales that are larger than those encountered in core-flooding experiments. The objective of this experiment was to demonstrate that nanoparticles can be transported over large distances without significant particle retention in the porous medium. Previous injection experiments in this project had been carried out in rock cores, which are significantly smaller than an actual reservoir. The $10 \mathrm{~m}$ long slim-tube represents an intermediate length-scale between core and reservoir scales. The calibration of pressure transducers used in experiments, gas and liquid permeability measurements, and the actual injection experiment are described in this chapter.

\subsection{Experimental Methods}

This experiment was performed in cooperation with colleague Mohammed Alaskar. Two $25 \mathrm{~m}$ stainless steel slim-tubes were constructed for high-temperature flow experiments, one of which is pictured in Figure 2.1a. Once nanosensors are developed that are proven to measure temperature in bench heating experiments, these steel slim-tubes will be used to evaluate their capabilities to flow and measure temperature. However, the steel tubes can be used for one experiment only, as they would then contain nanoparticles that would make subsequent experiments hard to interpret. Therefore, a disposable tube was sought for an initial experiment. For the purposes of this experiment (using inert nanoparticles at room temperature), a $10 \mathrm{~m}$ slim-tube was constructed by packing polypropylene tubing with sand of $1 \mathrm{~mm}$ maximum diameter and fitted with filter paper, screens, and valves at each end. This slim tube is pictured in Figure 2.1b. 

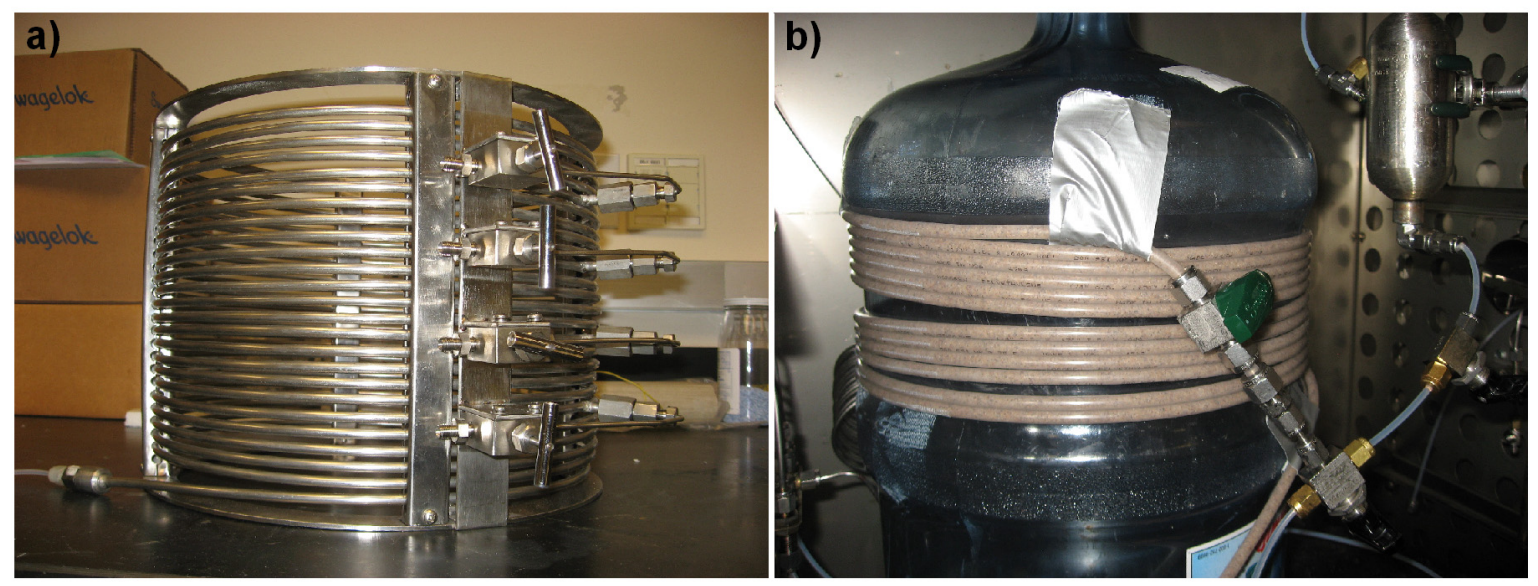

Figure 2-1: Photographs of (a) the $25 \mathrm{~m}$ stainless steel slim-tube and (b) the $10 \mathrm{~m}$ polypropylene slim tube.

\subsubsection{Transducer Calibration}

Four differential pressure transducers (Model DP15) manufactured by Validyne Engineering Corporation were calibrated for use in the slim tube experiment. A standard pressure gauge was used to calibrate transducers with ratings of $12.5,20,50$, and $125 \mathrm{psi}$. The signal sent by these transducers is measured in V, and each was calibrated such that atmospheric pressure corresponds to $0 \mathrm{~V}$ and its maximum pressure rating corresponds to $10 \mathrm{~V}$. The calibration plots for these transducers are shown in Figures 2-2 -2-5.

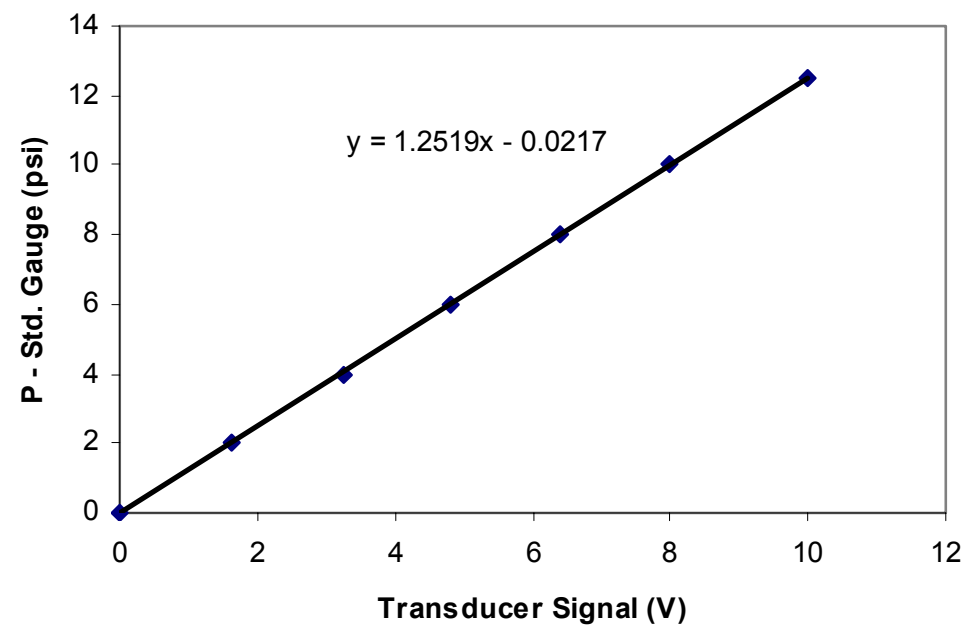

Figure 2-2: Calibration plot of 12.5 psi transducer 


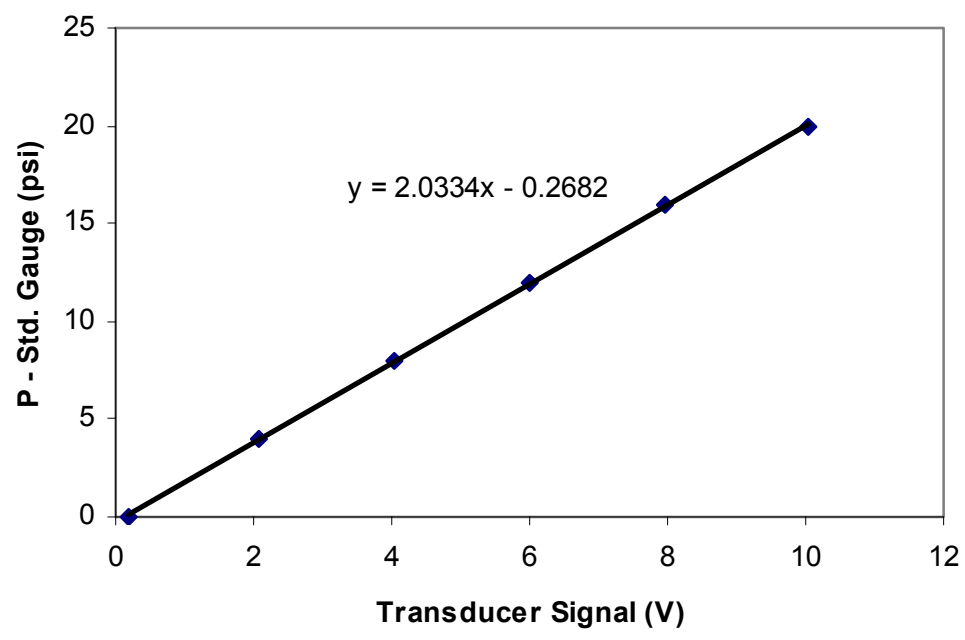

Figure 2-3: Calibration plot of 20 psi transducer

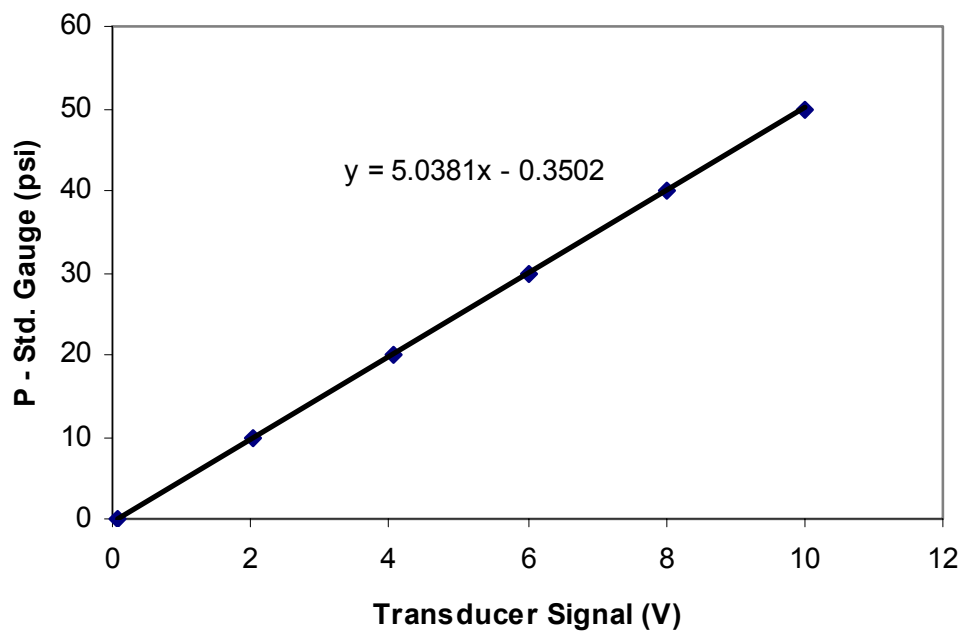

Figure 2-4: Calibration plot of 50 psi transducer 


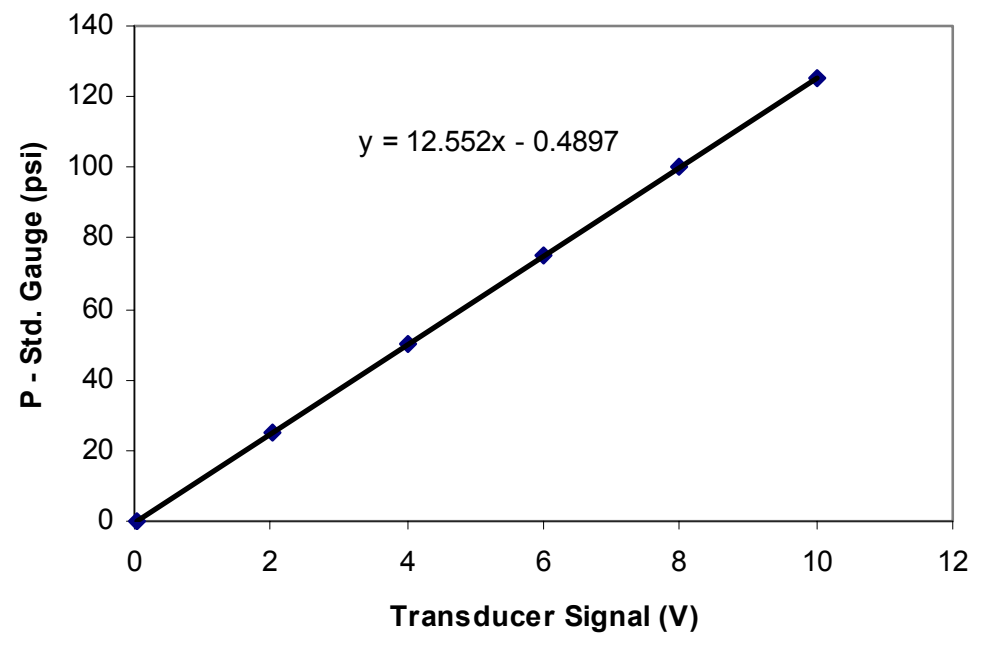

Figure 2-5: Calibration plot of 125 psi transducer

\subsubsection{Gas permeability measurement}

The gas permeability was measured, and the Klinkenberg effect (gas slippage) was considered to estimate the equivalent liquid permeability. The liquid permeability of the slim tube was then measured. The apparatus used in the measurement of gas permeability is shown in Figure 2-6. Nitrogen $\left(\mathrm{N}_{2}\right)$ was the gas used in this experiment. The inlet and outlet pressures were measured using differential pressure transducers of 125 and 50 psi ratings, respectively. The flow rate at the outlet was measured using a stop-watch and graduated cylinder.

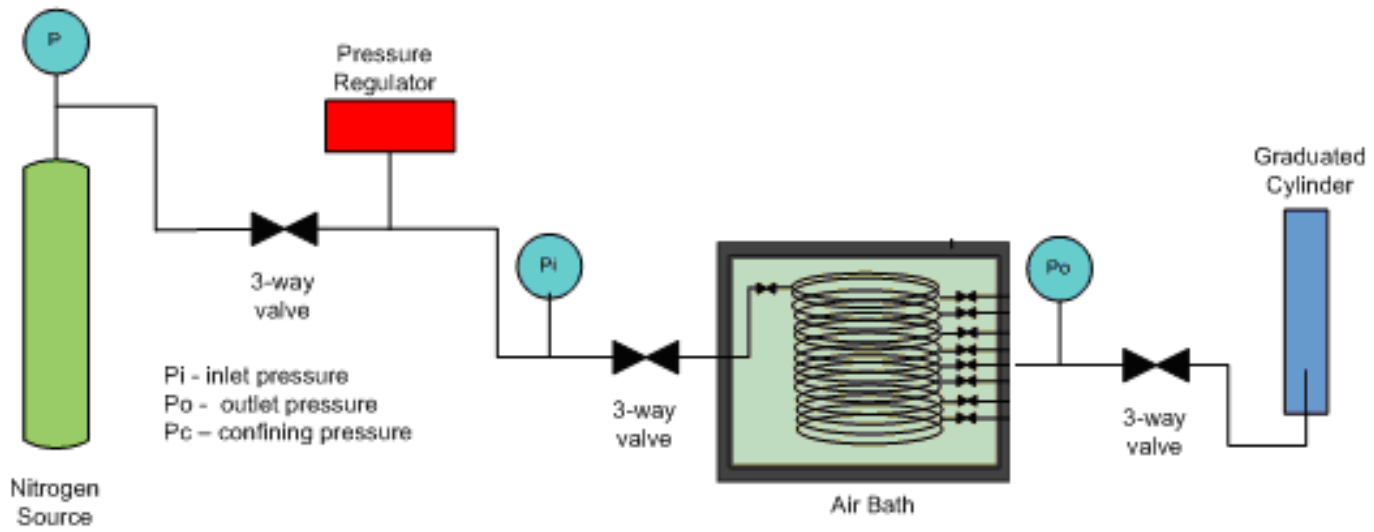

Figure 2-6: Schematic of the experimental apparatus used for the measurement of the gas permeability in the slim-tube.

The gas permeability measurement was performed by introducing $\mathrm{N}_{2}$ at different inlet pressures, which correspond to different flow rates. The average gas permeability was found to be around 50.2 darcy by applying Darcy's law for compressible fluids which is given by Equation 2-1. 


$$
k_{\text {gas }}=\frac{2 \mu p_{\text {out }} q L}{A\left(p_{\text {in }}^{2}-p_{\text {out }}^{2}\right)}
$$

where $k_{\text {gas }}$ is gas permeability, $\mu$ is the gas viscosity, $q$ is the gas flowrate, $L$ is the length of the flow-path, $A$ is the cross-sectional area of the flow-path, and $p_{\text {in }}$ and $p_{\text {out }}$ are the inlet and outlet pressures, respectively. The gas permeability measurements are plotted as a function of the reciprocal of mean pressure in Figure 2-7.

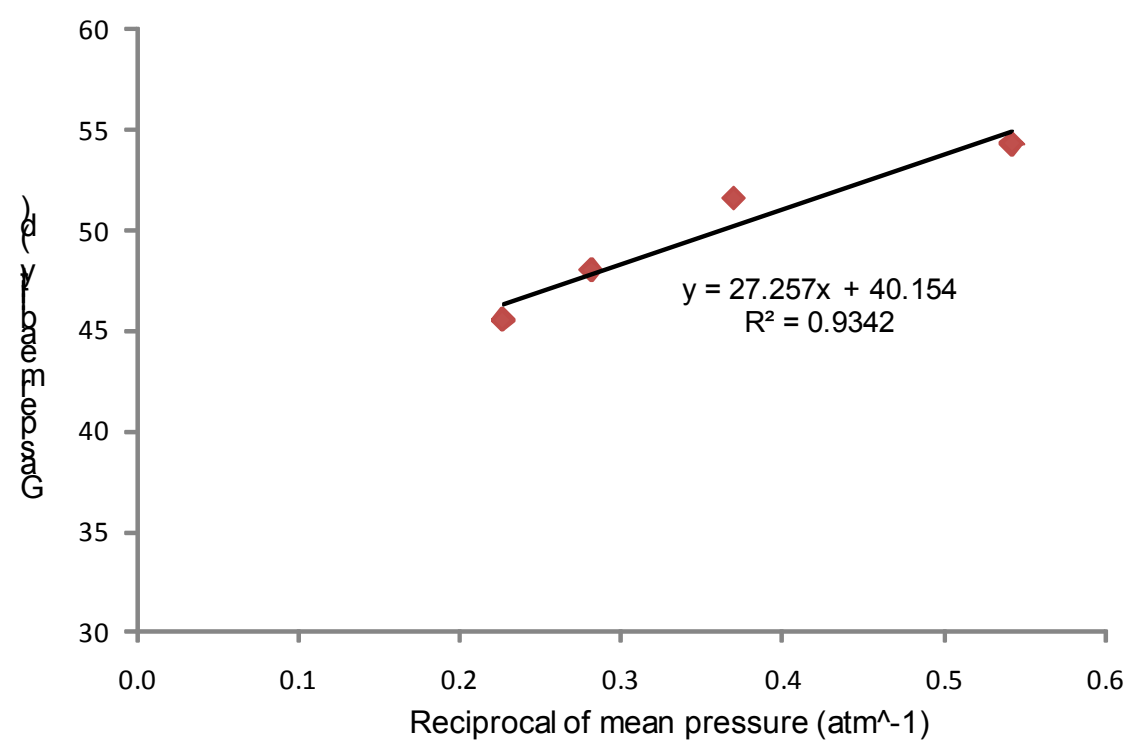

Figure 2-7: Gas permeability versus the reciprocal of mean pressure

In order to consider Klinkenberg effect and predict the liquid permeability of the slimtube, linear least-squares regression was used to correlate the permeability measurements to the reciprocal of mean pressure. The line was then extrapolated to its intercept with the permeability axis (which represents infinite mean pressure or zero reciprocal of mean pressure). The permeability value at this intercept can be designated as the equivalent liquid permeability (Amyx, Bass, and Whiting, 1960). As shown in Figure 2-7, the average equivalent liquid permeability is 40.2 darcy for these measurements.

\subsubsection{Liquid permeability measurement}

The liquid permeability of the slim-tube was also measured. A schematic of the apparatus used in the measurement of liquid permeability is shown in Figure 2-8. 


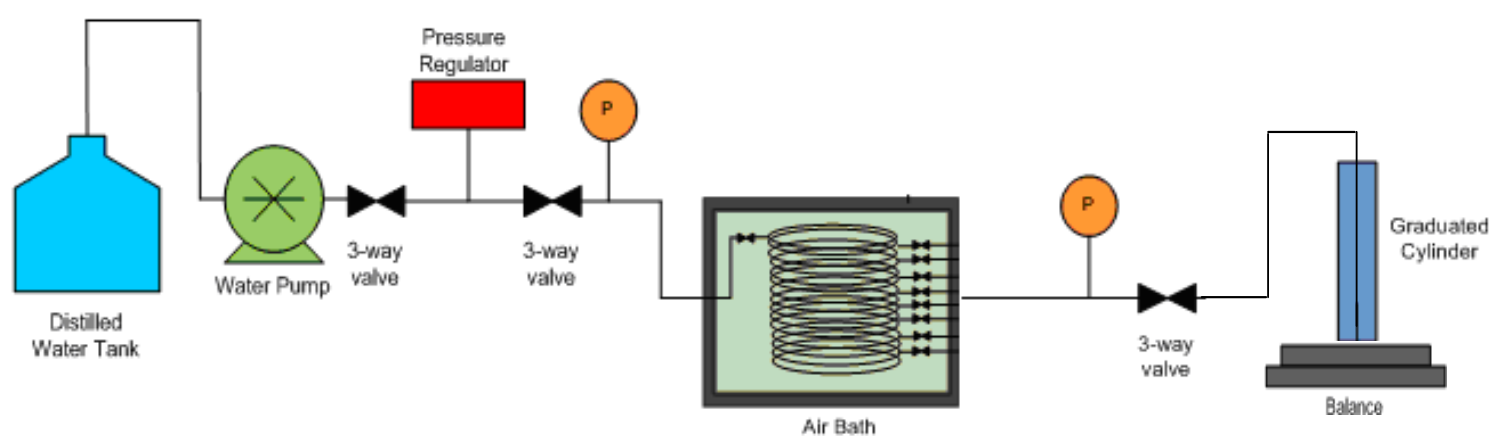

Figure 2-8: Schematic of the experimental apparatus used for the measurement of the liquid permeability in the slim-tube

The apparatus was evacuated using a Welch Vacuum Pump for 4 hours at a vacuum pressure of about 25 mTorr to remove moisture. A column of pure water with a known weight $\left(W_{d}\right)$ was introduced to saturate the entire sand-packed and inlet tubes. The new water column weight $\left(W_{s}\right)$ was then noted. The porosity and pore volume of the slimtube were measured to be $35.5 \%$ and $51.9 \mathrm{~cm}^{3}$, respectively. The porosity calculation was performed according to Equations 2-2 through 2-6.

$$
\begin{gathered}
\phi=\frac{V_{p}}{V_{B}} * 100 \\
V_{p 1}=\frac{W_{s}-W_{d}}{\rho_{w}} \\
V_{p 2}=\pi r_{t}^{2} l_{t} \\
V_{p}=V_{p 1}-V_{p 2} \\
V_{B}=\pi r^{2} l
\end{gathered}
$$

where $\phi$ is the porosity in percentage, $V_{p}$ and $V_{b}$ are pore and bulk volumes of sandpacked tube, respectively, $V_{p 1}$ is the total volume of the entire apparatus (including the sand-packed tube and dead volume associated with the inlet tubes), $V_{p 2}$ is the dead volume of inlet tubes, $W_{s}$ and $W_{d}$ are the weight of water column after and before saturation, respectively, $\rho_{w}$ is the density of water, $r$ and $l$ are the radius and length of the sand-packed tube, respectively, and $r_{t}$ and $l_{t}$ are the radius and length of the inlet tubes, respectively.

The same differential pressure transducers were used as previously in the gas permeability measurement. In addition, a water pump was used to inject pure water and the flow rate measured using a stop-watch and a Mettler balance (Model PE 300).

To measure the liquid permeability, deaerated water was injected at several flow rates ranging from 1 to $3 \mathrm{ml} / \mathrm{min}$ at different differential pressures. The average liquid permeability was measured to be 49.9 darcy. Darcy's law for horizontal flow was utilized to compute the permeability. Darcy's law for horizontal flow is given in Equation 2-7. 


$$
k_{\text {liq }}=\frac{q \mu L}{A \Delta p}
$$

where $k_{\text {liq }}$ is the liquid permeability, $q$ is the volumetric flowrate, $\Delta p$ is the differential pressure across the slim-tube, $\mu$ is the viscosity, $L$ and $A$ are the length and the crosssectional area of the slim tube, respectively.

\subsubsection{Slim-tube injection experiment}

The experimental apparatus used to inject nanoparticles into the slim-tube is shown in Figures 2-9 and 2-10.

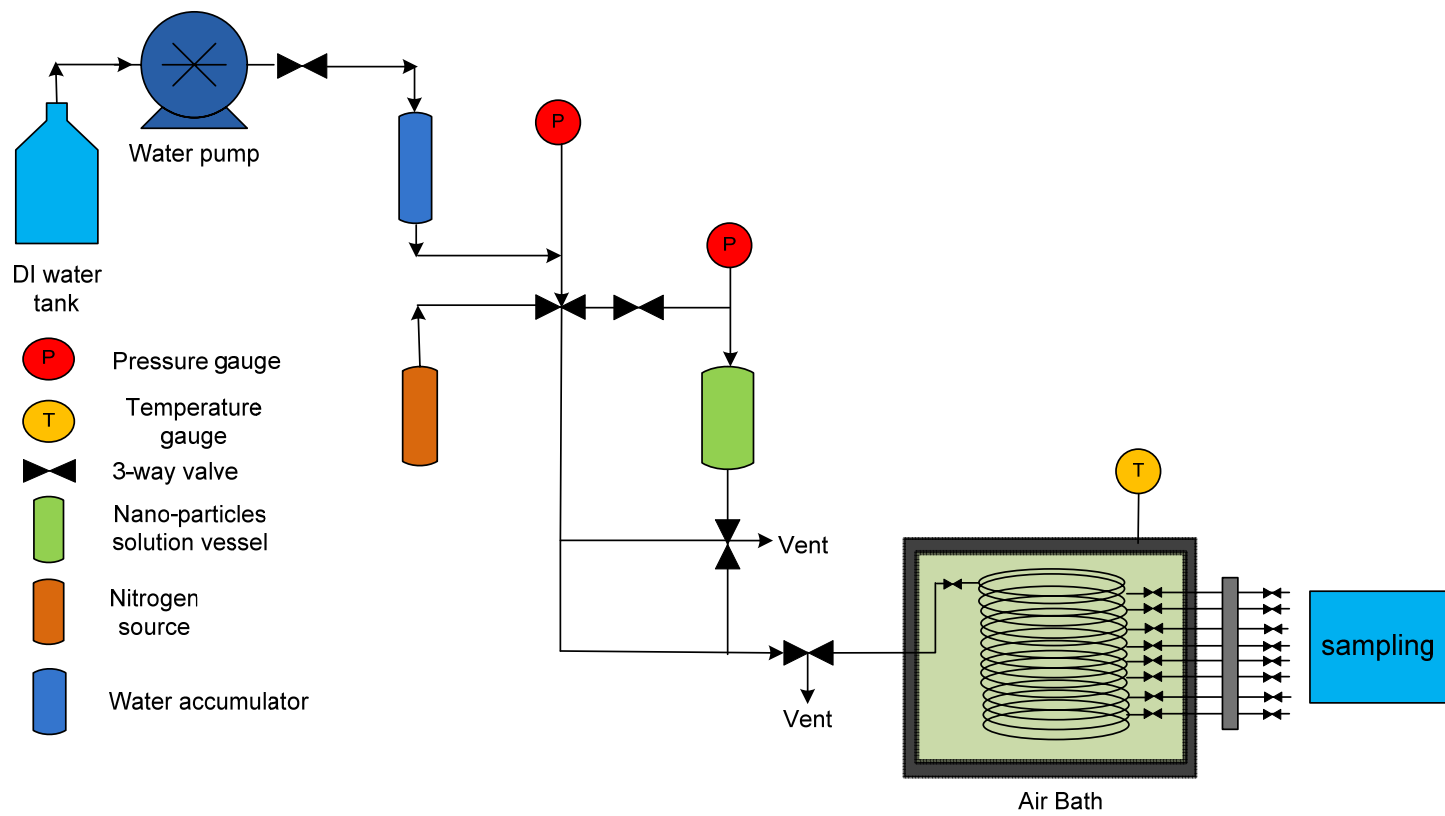

Figure 2-9: Schematic of the experimental apparatus used for the nanoparticle injection experiment in the slim-tube. 


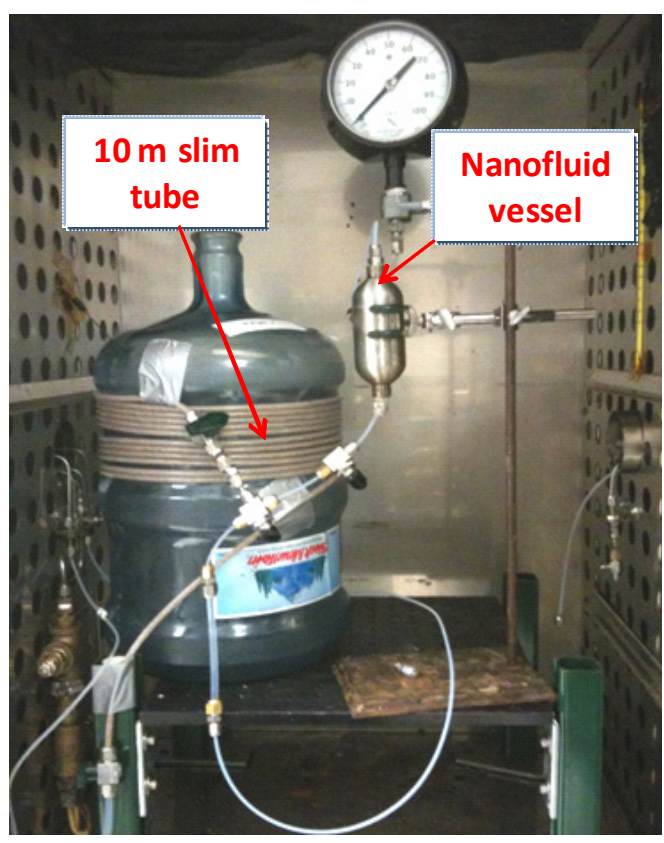

Figure 2-10: Photograph of apparatus used for the nanoparticle injection experiment in the slimtube.

$\mathrm{SiO}_{2}$ (silica) nanoparticles prepared by Steve Connor were used in this injection. Mohammed Alaskar characterized the nanoparticles using Dynamic Light Scattering (DLS) and Scanning Electron Microscopy (SEM), as shown in Figures 2-11 and 2-12.

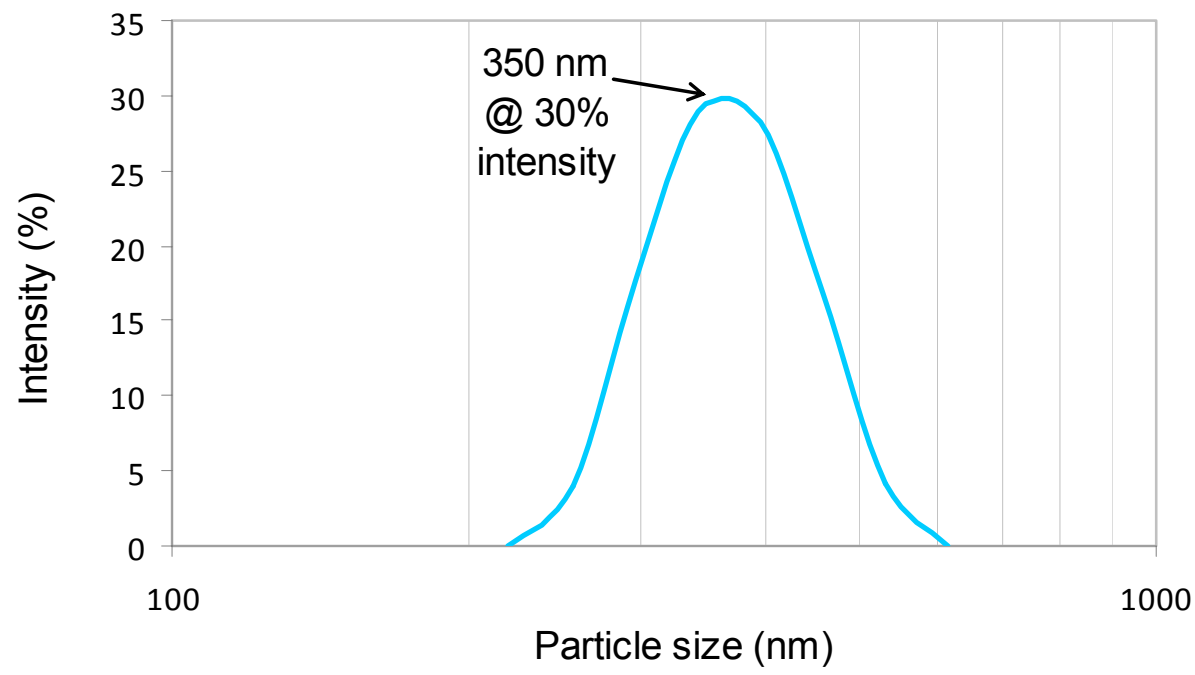

Figure 2-11: Size distribution of influent silica nanoparticles as measured by DLS. 


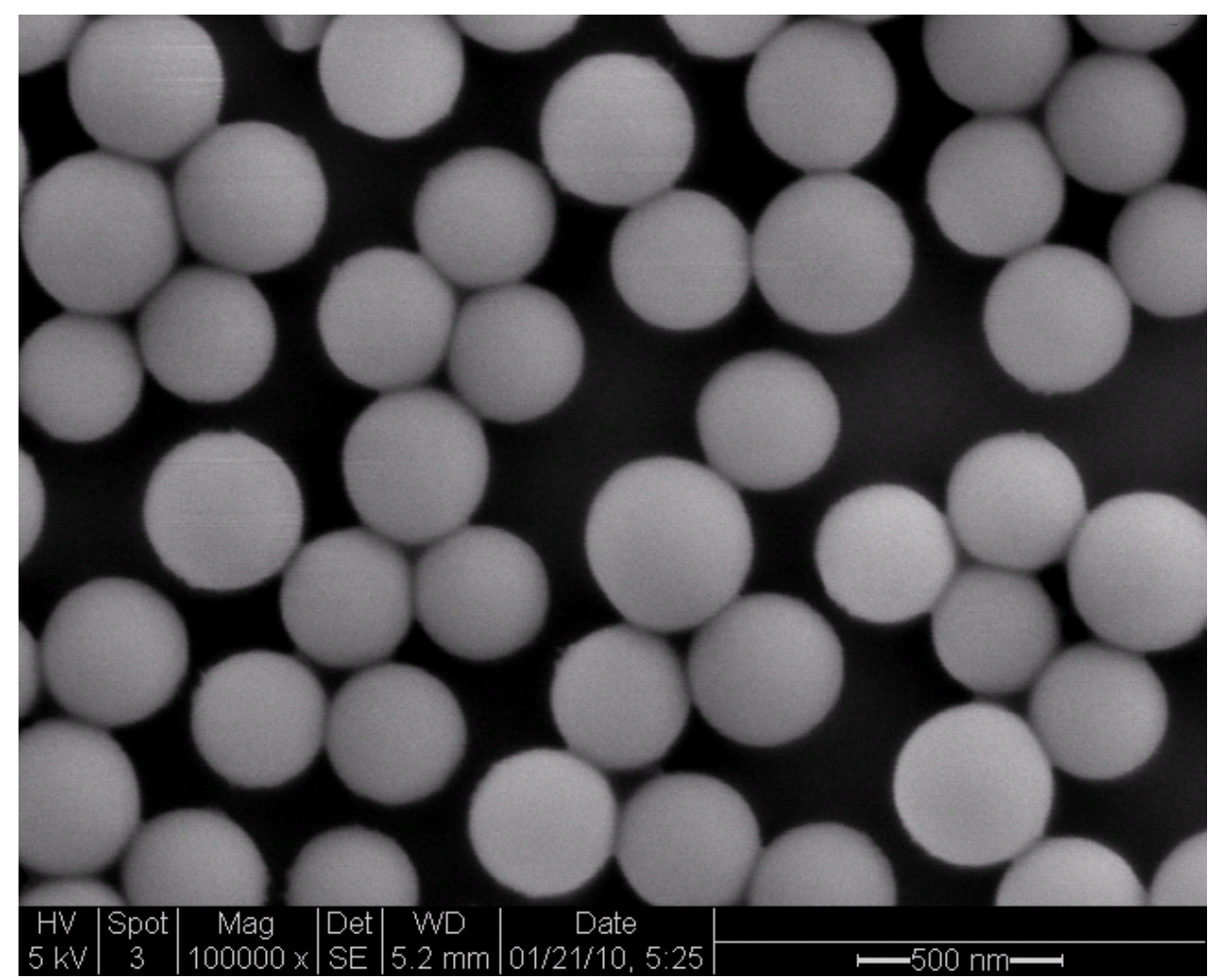

Figure 2-12: SEM image showing a sample of influent silica nanoparticles used in injection.

As shown in Figures 2-10 and 2-11, the silica nanoparticles were spherical in shape and relatively monodisperse, with a modal diameter of $350 \mathrm{~nm}$. Prior to injection of the nanofluid, the apparatus was preflushed with 4 pore volumes of pure water. $10 \mathrm{ml}$ of nanofluid was injected (about $20 \%$ of 1 pore volume), then pure water was injected continuously, and effluent samples were collected and characterized using DLS and SEM imaging. Sampling was most frequent during injection of the first 2 pore volumes in order to capture differences in concentration. After 9 pore volumes of water were injected, a reverse flushing experiment was carried out, in which 5 pore volumes of water were injected.

\subsection{Results}

Silica nanoparticles were detected visually in the effluent following the injection of about 0.5 pore volumes of water. After 2 pore volumes had been injected, no particles could be detected visually, so sampling and measurements were performed less frequently. The increasing concentration of nanoparticles during the first 2 pore volumes of injection is illustrated in Figure 2-13. The cloudy samples have high concentrations of silica nanoparticles compared to semitransparent samples. Nanoparticles were also detected in the effluent samples using DLS and SEM imaging, confirming that they were transported successfully through the $10 \mathrm{~m}$ slim-tube. These results are shown in Figures 2-13 through 2-15. Note that the SEM images in Figure 2-14 agree with the assessment of concentration made in Figure 2-13. 


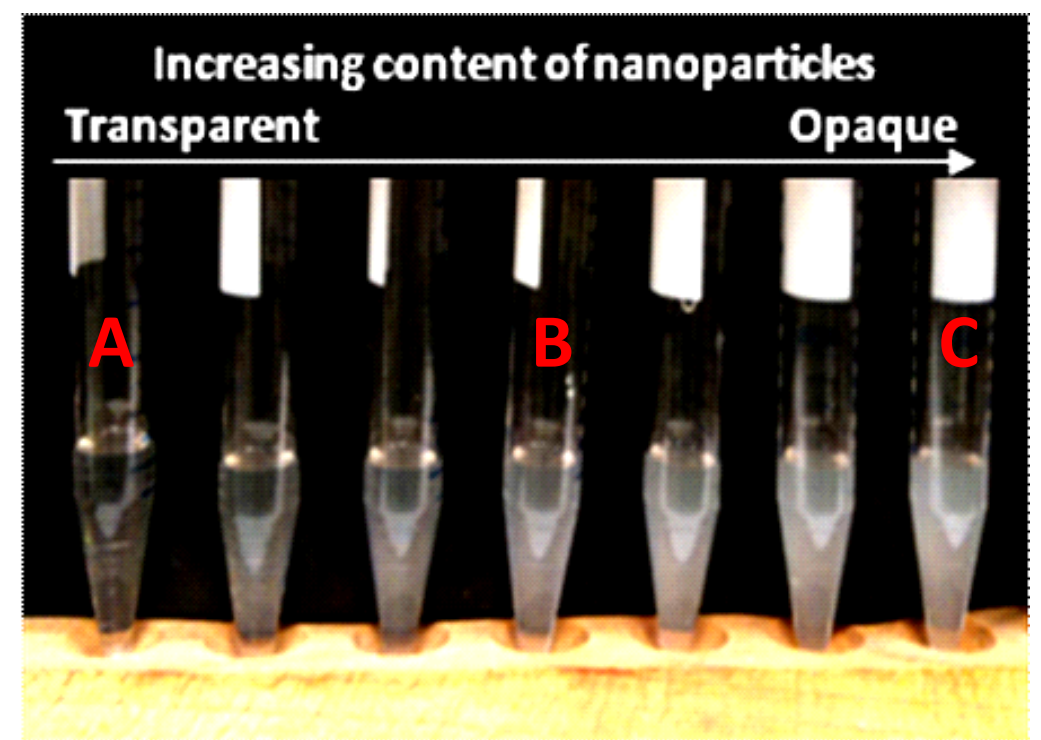

Figure 2-13: Photograph of effluent samples in order of collection. Note that the cloudy or opaque samples are more concentrated with silica nanoparticles.
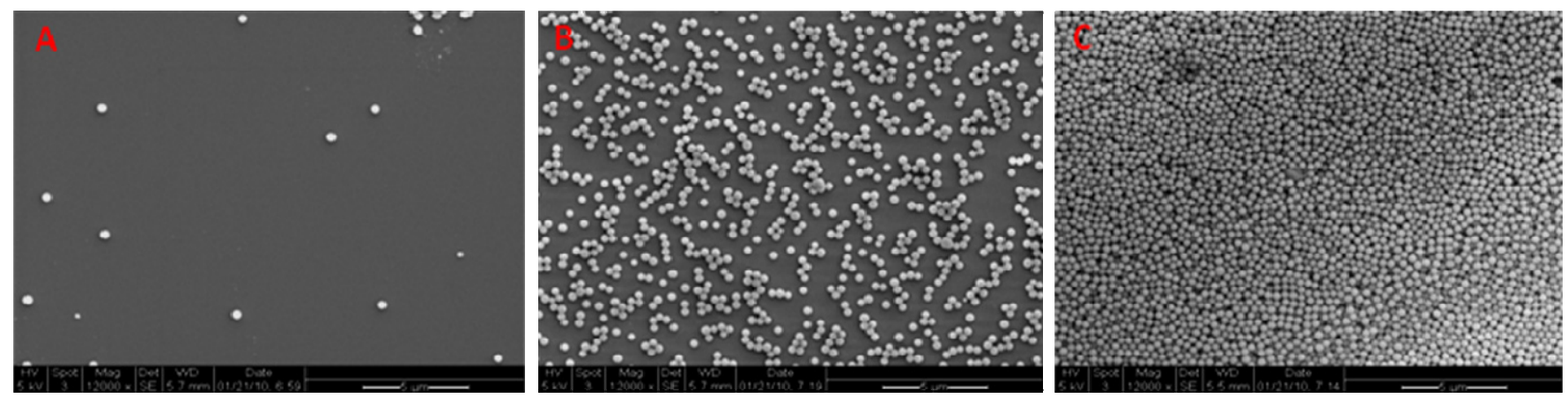

Figure 2-14: SEM images of silica nanoparticles in effluent samples. Note that these images correspond to the samples shown in Figure 2-13 with the same labels. 


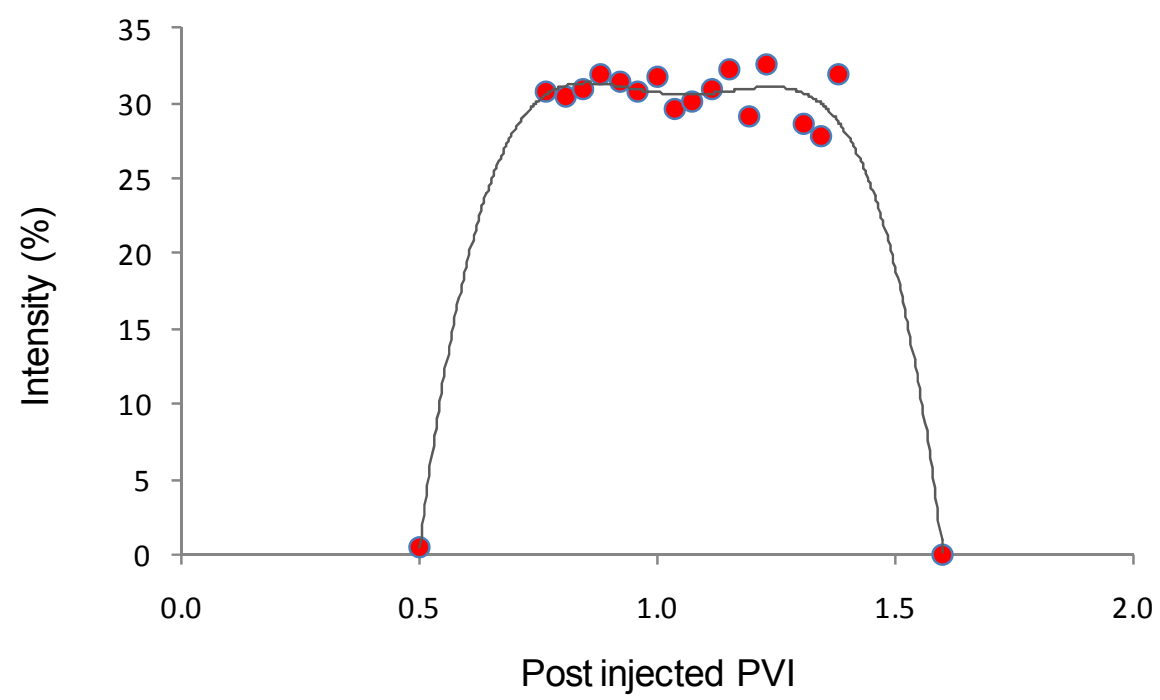

Figure 2-15: DLS results of effluent samples. Intensity at $350 \mathrm{~nm}$ diameter is shown, indicating that detectable amounts of nanoparticles were present in the effluent from 0.5 to 1.6 pore volumes.

The permeability fluctuated slightly during the injection, but remained fairly constant around $50 \pm 4$ darcy. The permeability measurements taken during the injection and backflushing experiments are plotted in Figure 2-16.

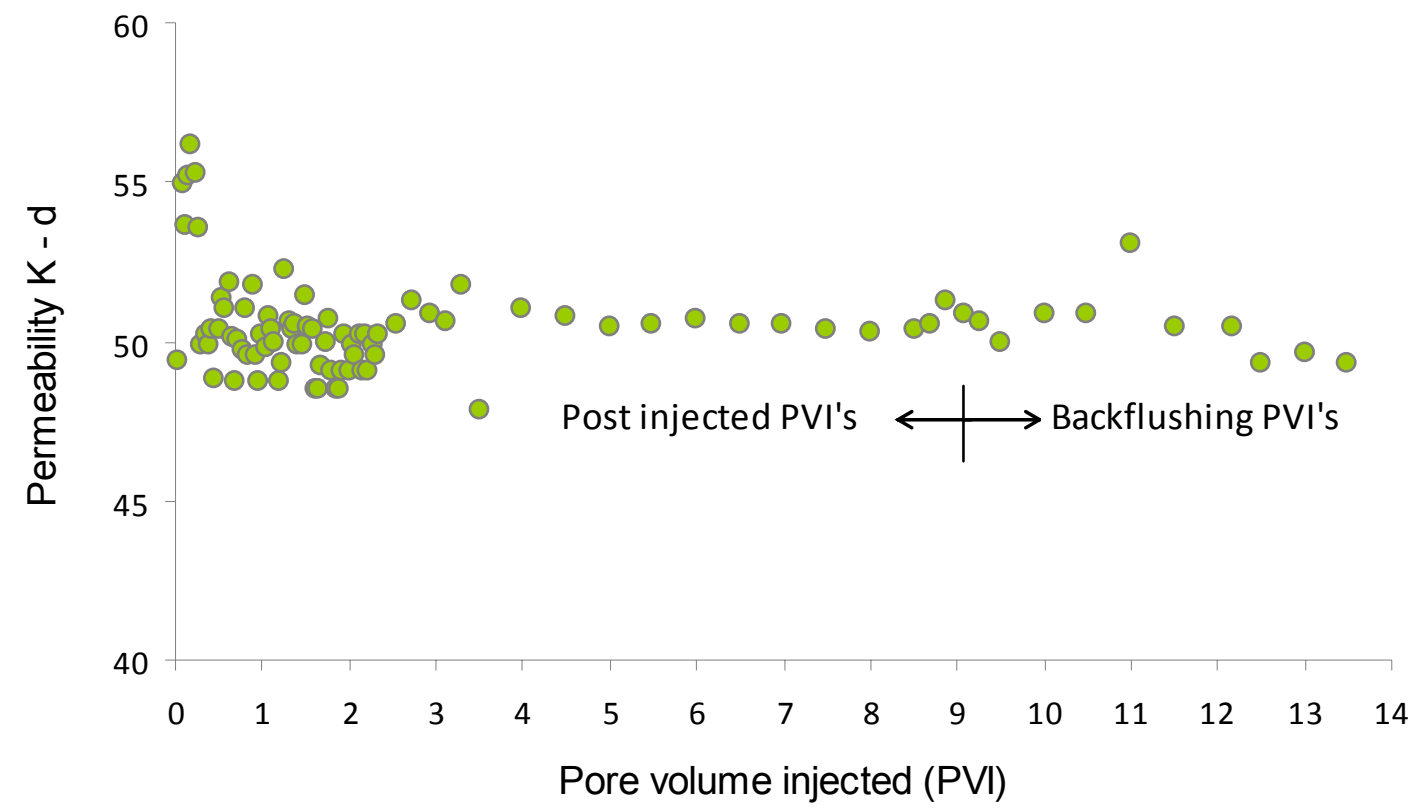

Figure 2-16: Permeability measurements taken during the silica nanoparticle injection and backflushing experiments. 
A reliable method to measure silica nanoparticle concentration has not been identified at this time. SEM images from later pore volumes indicate that even though nanoparticles could not be detected by DLS, they were still present in the effluent at low concentrations, as shown in Figure 2-17.
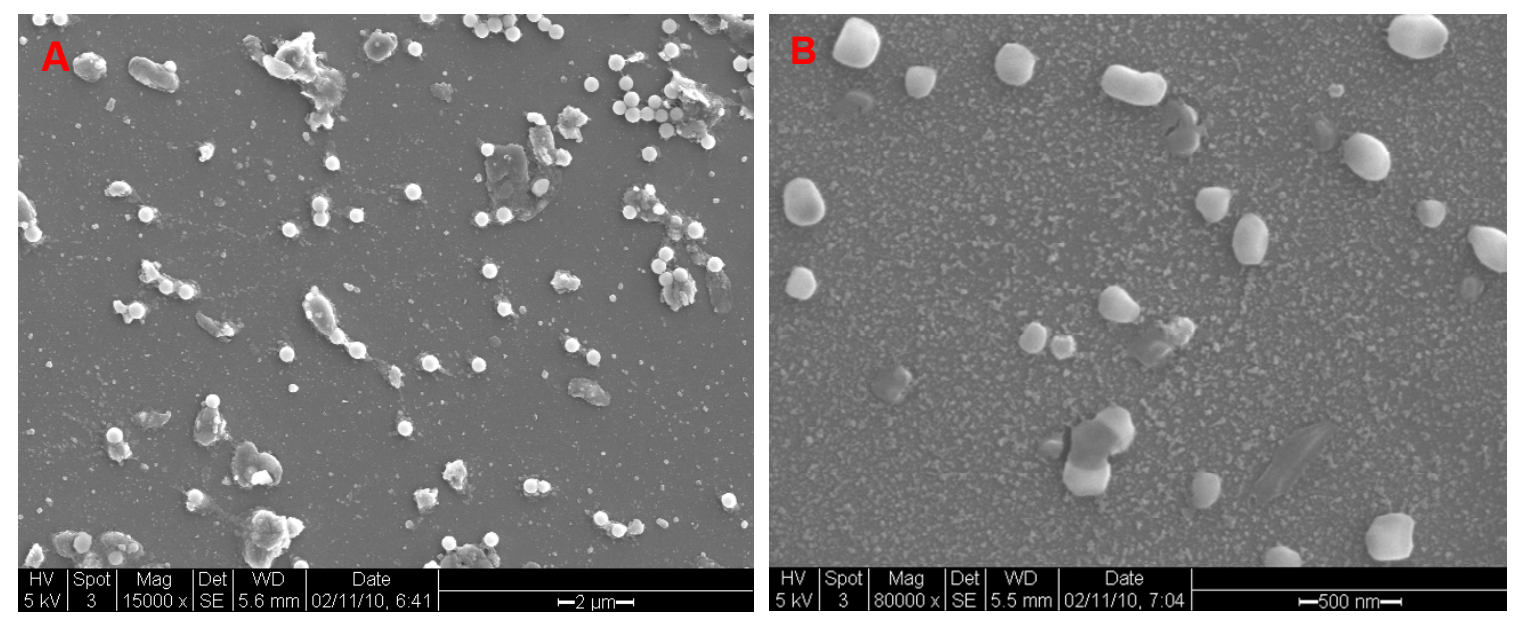

Figure 2-17: SEM images of effluent samples taken from the (a) $3^{\text {rd }}$ and (b) $8^{\text {th }}$ injected pore volumes.

Analysis of samples taken during backflushing indicate that some particles were trapped in the slim-tube or the inlet tubing, as shown in Figure 2-18.

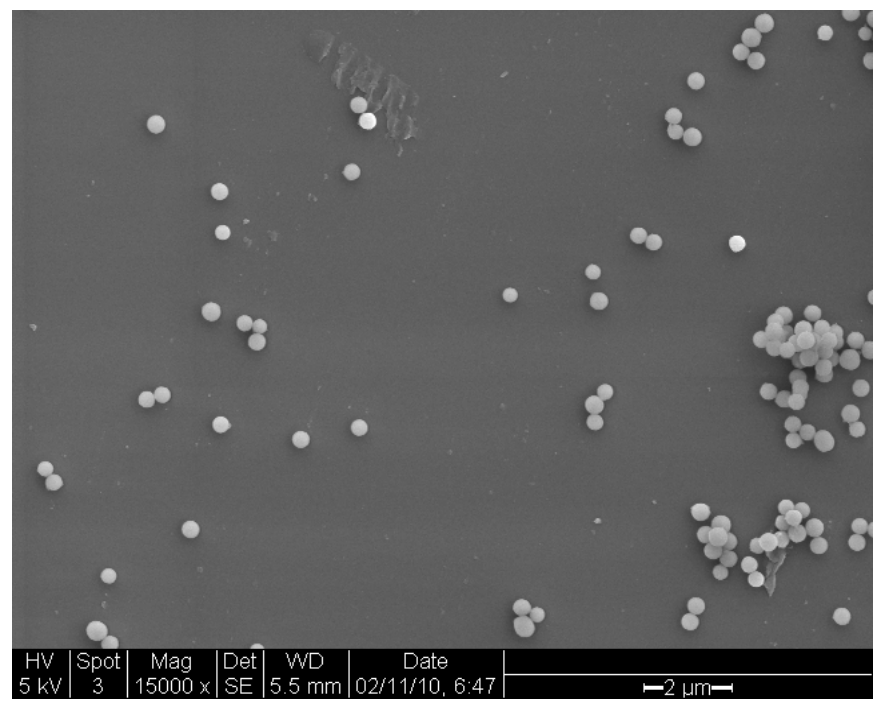

Figure 2-18: SEM image of effluent sample taken from the $1^{\text {st }}$ pore volume of backflushing.

The encouraging results of this injection experiment show that nanoparticles can be transported through porous media with length scales larger than those in rock cores. It is also apparent that silica nanoparticles have good transport properties for this application, and thus, this material has potential to be incorporated into various geothermal reservoir sensing schemes. 



\section{Chapter 3}

\section{Magnetic Collection of Nanoparticles}

For nanosensors to provide information about temperature distribution in a geothermal reservoir, they must be successfully collected from produced fluid. As this is not a trivial task, an experiment was performed to investigate the concept of magnetic collection of paramagnetic nanoparticles from flowing fluid. In order for a magnetic collection scheme to be successful, the magnetic force on the particles must dominate inertial, drag, gravitational, and diffusion forces acting on the particles (Moeser et al., 2004). In geothermal applications, it is likely that the two main competing forces will be the magnetic and inertial forces on the particles. Gerber and Birss (1983) define the magnetic force on a particle in a magnetic field as:

$$
F_{m}=\mu_{0} V_{p} M_{p} \bullet \nabla H
$$

where $F_{m}$ is the magnetic force, $\mu_{0}$ is the constant permeability of free space, $V_{p}$ is the volume of the paramagnetic particle, $M_{p}$ is the magnetization of the particle, and $\nabla H$ is the magnetic field gradient. It is clear from Equation 3-1 that there are three parameters that can be tuned in order to increase the magnetic force on the particles: particle size, magnetization (which can be tuned by changing particle composition), and applied magnetic field gradient. It seems as if the applied magnetic field gradient may have the most potential as a tuning knob, because particle size and composition are constrained by other factors (e.g. mobility in the reservoir).

In this proof of concept experiment, it was decided to use low flowrates (i.e. low pressure gradient and inertial forces) were used in order to increase the likelihood of successful collection. The results of this experiment will be used to help design future experiments.

\subsection{Experimental Methods}

The experimental apparatus used for magnetic nanoparticle collection is illustrated in Figure 3-1. 


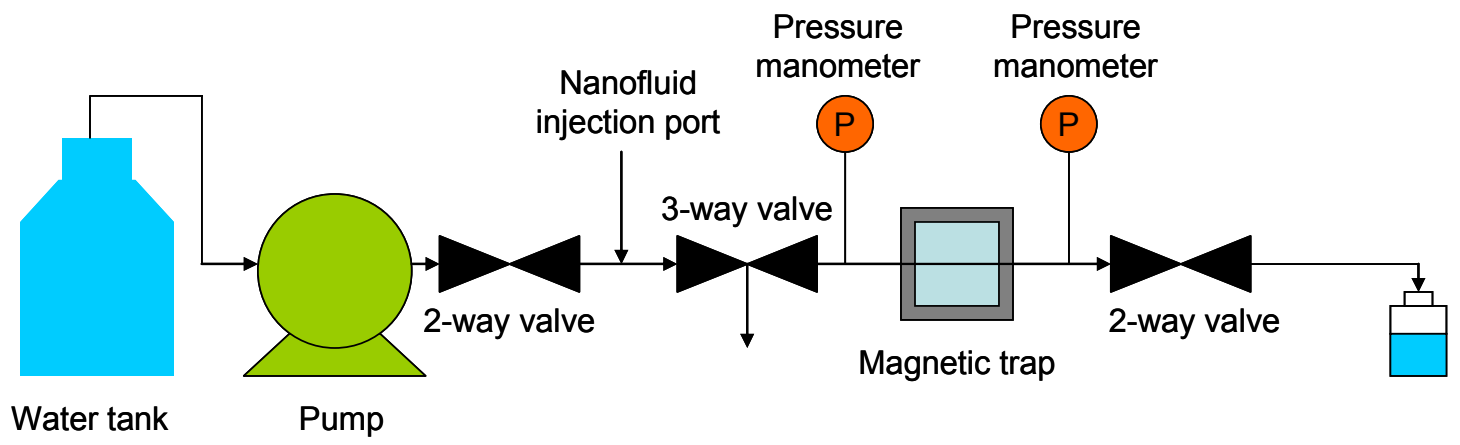

Figure 3-1: Schematic of experimental apparatus used in the magnetic collection experiment

A Dynamax solvent delivery system (Model SD-200) was used to pump water through the apparatus. Four BX084 neodymium block magnets manufactured by K\&J Magnetics were used in the magnetic trap, with two magnets affixed to each side of the tube. This configuration was chosen in order to expose the injected nanoparticles to the strongest part of the magnetic field, which is illustrated in Figure 3-2. The proximity of the magnets is critical to the effectiveness of the collection scheme because magnetic force is a strong function of distance, as shown in Figure 3-3 for the particular magnets used.

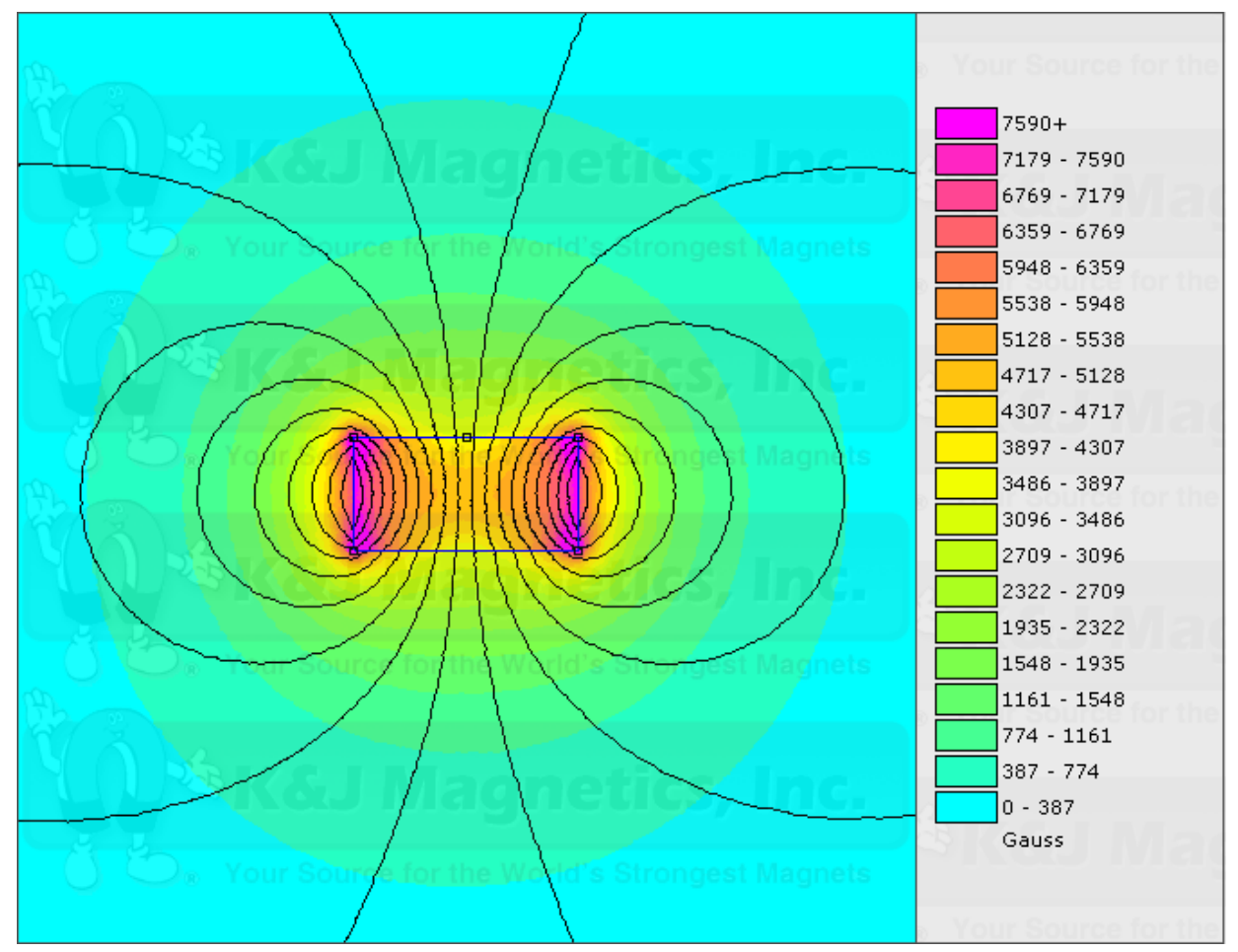

Figure 3-2: Magnetic field of neodymium block magnets. Reproduced from K\&J Magnetics. 


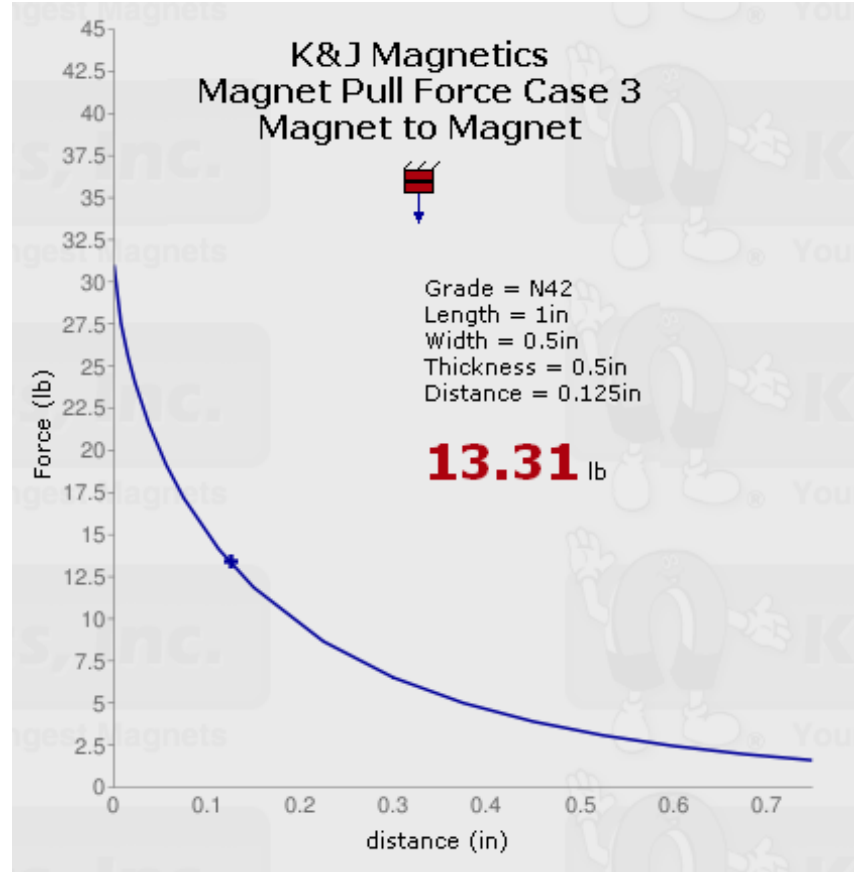

Figure 3-3: Magnetic pull force between two neodymium magnets as a function of distance. The point on the curve corresponds to $13.31 \mathrm{lb}_{\mathrm{f}}$ at a distance of $0.125 \mathrm{in}$., or the radius of the collection tube used. Reproduced from K\&J Magnetics.

A $5 \mathrm{wt} \%$ suspension of paramagnetic iron oxide nanoparticles coated with silica in water were purchased from Corpuscular, Inc. These particles were characterized using SEM imaging, as shown in Figure 3-4. 


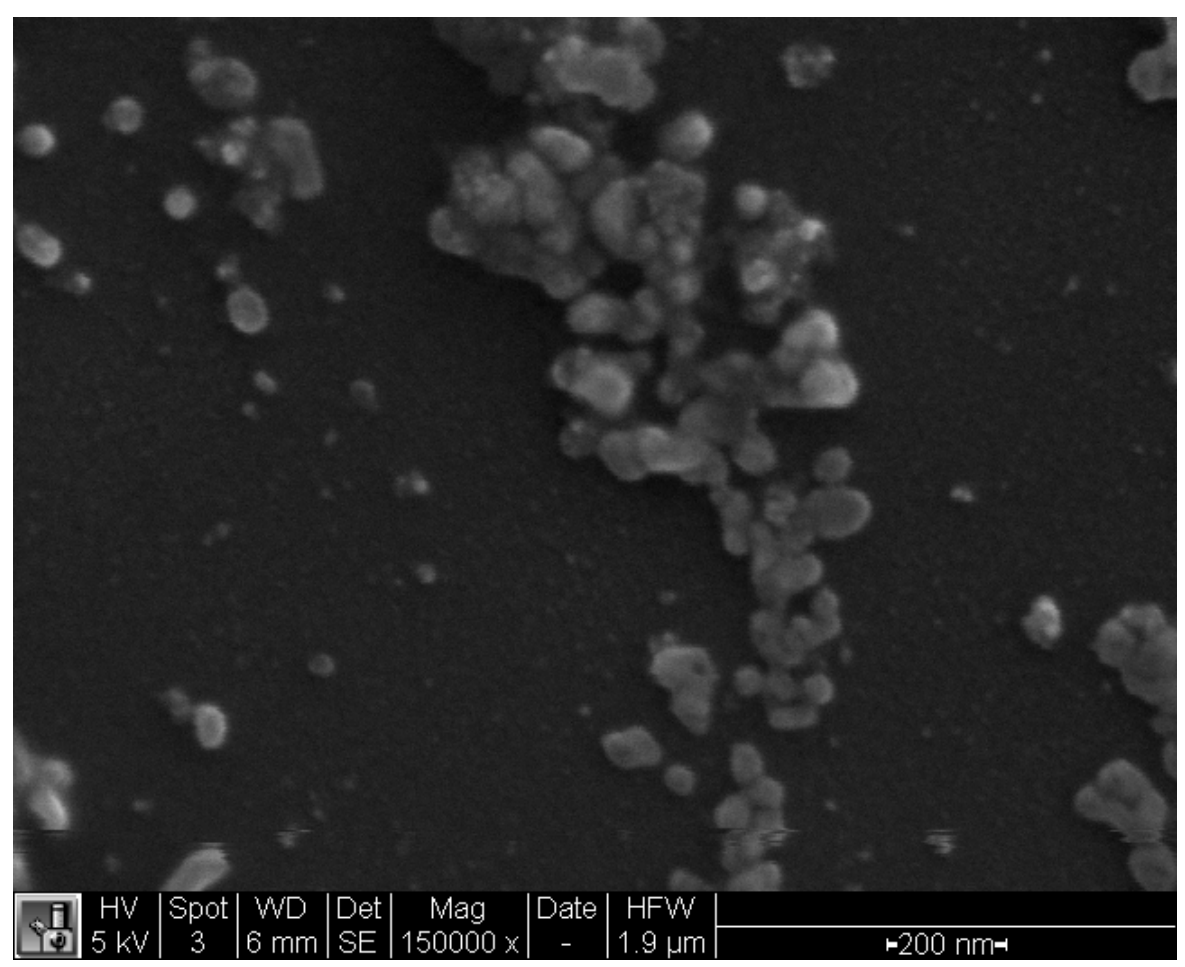

Figure 3-4: SEM image of iron oxide nanoparticles coated with silica.

The pump was used to fill the apparatus with water, the first valve was closed, the threeway valve was turned to allow water to be flushed out, and $1 \mathrm{ml}$ of iron oxide nanofluid was injected. The valves were turned to their initial settings, water was through the apparatus at a flowrate of $0.1 \mathrm{ml} / \mathrm{min}$ for 6.5 hours to push the nanofluid through the magnetic trap. All effluent was collected. Pressure remained constant throughout the experiment at $0.023 \mathrm{psig}$ and $0.018 \mathrm{psig}$ at the inlet and outlet of the collection tube, respectively. Most of the nanofluid was observed visually passing through the magnetic trap without being trapped. After flushing the apparatus with about $39 \mathrm{ml}$ of water, the effluent was very dilute, and a small static cloud of nanofluid was observed at the outlet of the magnetic trap.

At this point, the flowrate was increased to $2.0 \mathrm{ml} / \mathrm{min}$, and tendrils of previously static nanofluid were observed exiting the magnetic trap. The apparatus was flushed at this flowrate for about $50 \mathrm{~min}$, at which point the effluent was visually indistinguishable from water. The pump was turned off, the valves were closed, and the magnetic trap was removed, revealing a cloud of nanoparticles that had been trapped by the magnet, as shown in Figure 3-5. 


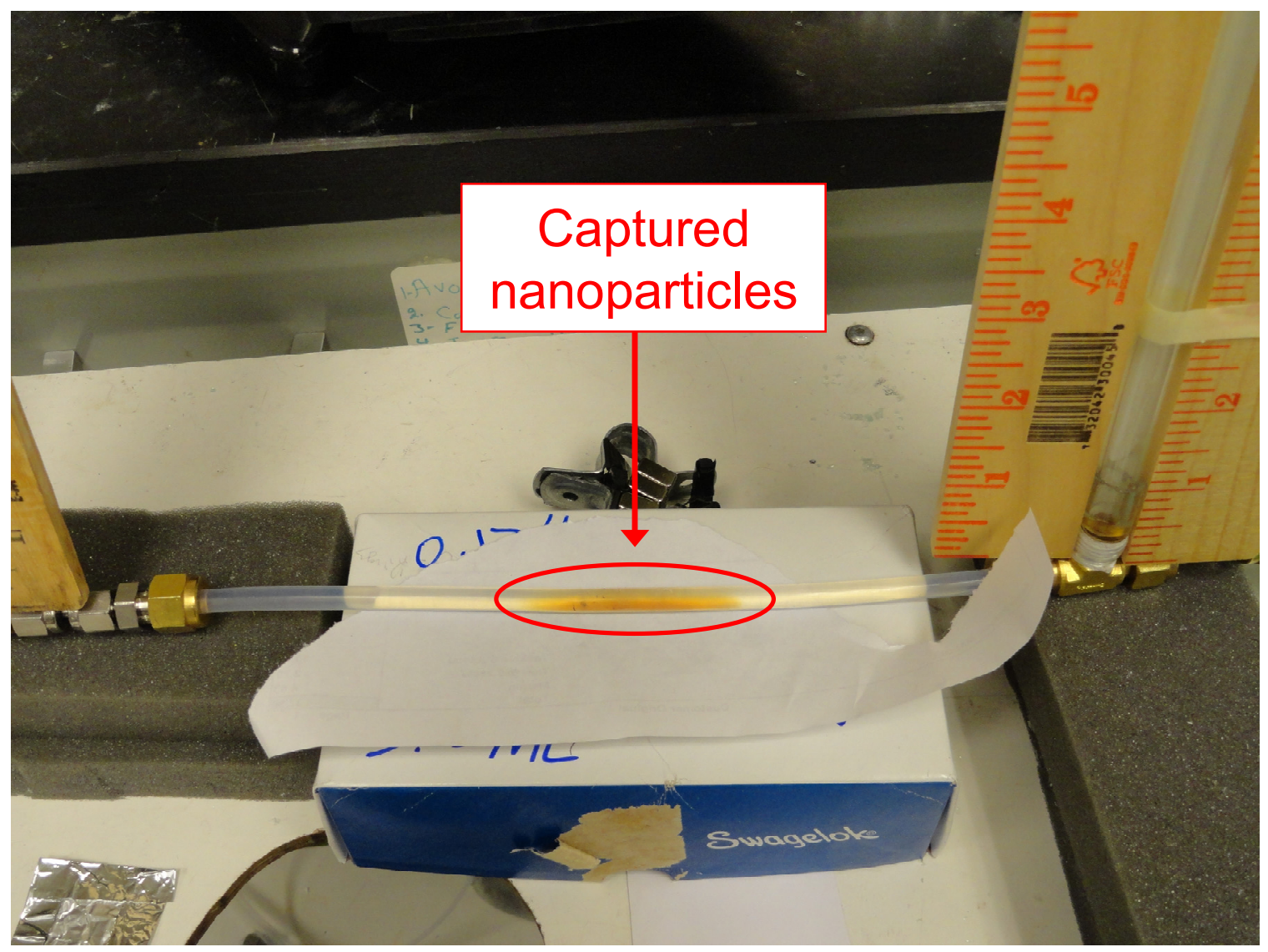

Figure 3-5: Photograph of trapped nanoparticles after the removal of the magnetic trap.

The outlet side of the collection tube was disconnected, and the previously trapped nanoparticles were collected. The volume of the collected fluid was measured to be 3.7 $\mathrm{ml}$. In order to calculate collection efficiency, the concentration of the nanoparticles in the trapped sample had to be estimated. This was done by using uv-vis spectroscopy to measure the absorbance spectra of the trapped sample and of dilutions prepared from the original nanofluid with known concentrations. The absorbance at a specific wavelength was found to scale linearly with concentration, and the resulting correlation was used estimate the concentration of the collected sample. The baseline concentration of nanoparticles was calculated to be $0.08 \mathrm{~g} / \mathrm{ml}$ using the $5 \mathrm{wt} \%$ specified by the manufacturers and the measured nanofluid density of $1.6 \mathrm{~g} / \mathrm{ml}$.

\subsection{Results}

In order to calculate the collection efficiency, or the percentage of injected nanoparticles that were remained in the magnetic trap at the end of the experiment, dilutions of the original nanofluid with known concentrations. The dilution with the closest visual match to the trapped nanofluid is shown in Figure 3-6. 


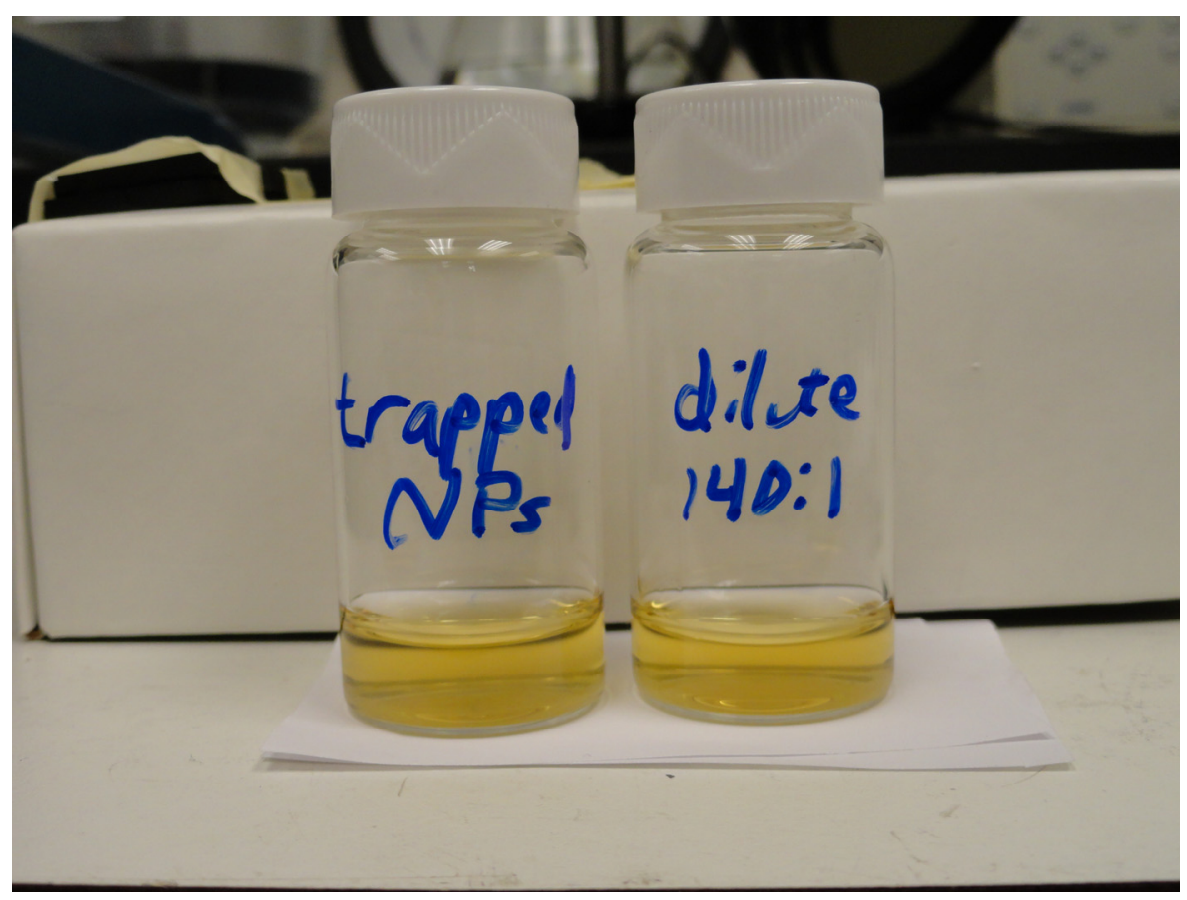

Figure 3-6: Visual comparison of trapped nanofluid sample and 142.5 to 1 dilution of original nanofluid.

The absorbance spectra of the trapped nanofluid and the prepared dilutions were measured using uv-vis spectroscopy, and are shown in Figure 3-7. As is predicted by Beer's Law, concentration was found to scale linearly with absorbance. This is shown in Figure 3-8. For this correlation, the absorbance was read at a wavelength of $460 \mathrm{~nm}$, because the spectra have an inflection point at this wavelength. 


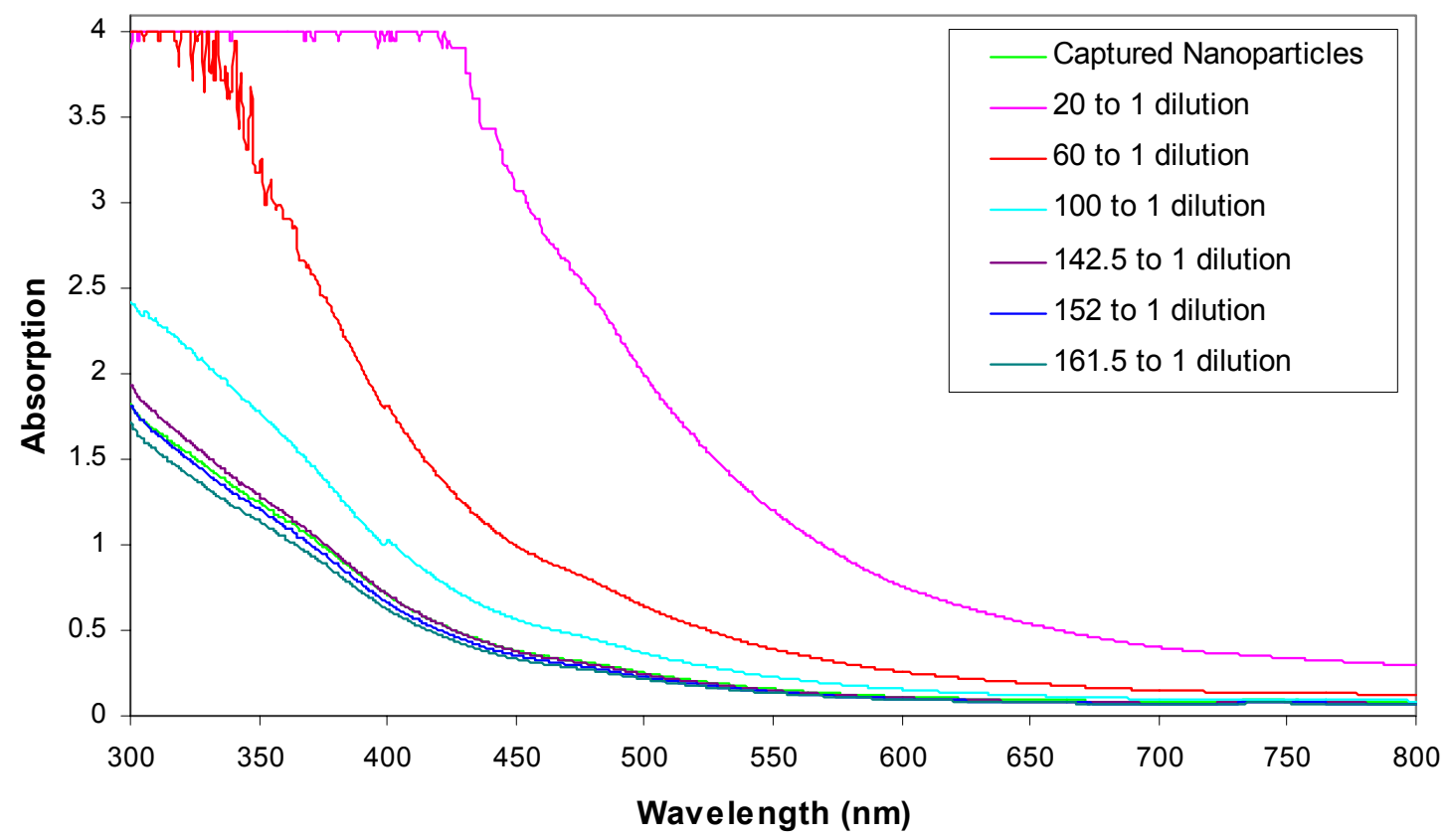

Figure 3-7: Absorbance spectra of suspensions of iron oxide nanoparticles coated with silica.

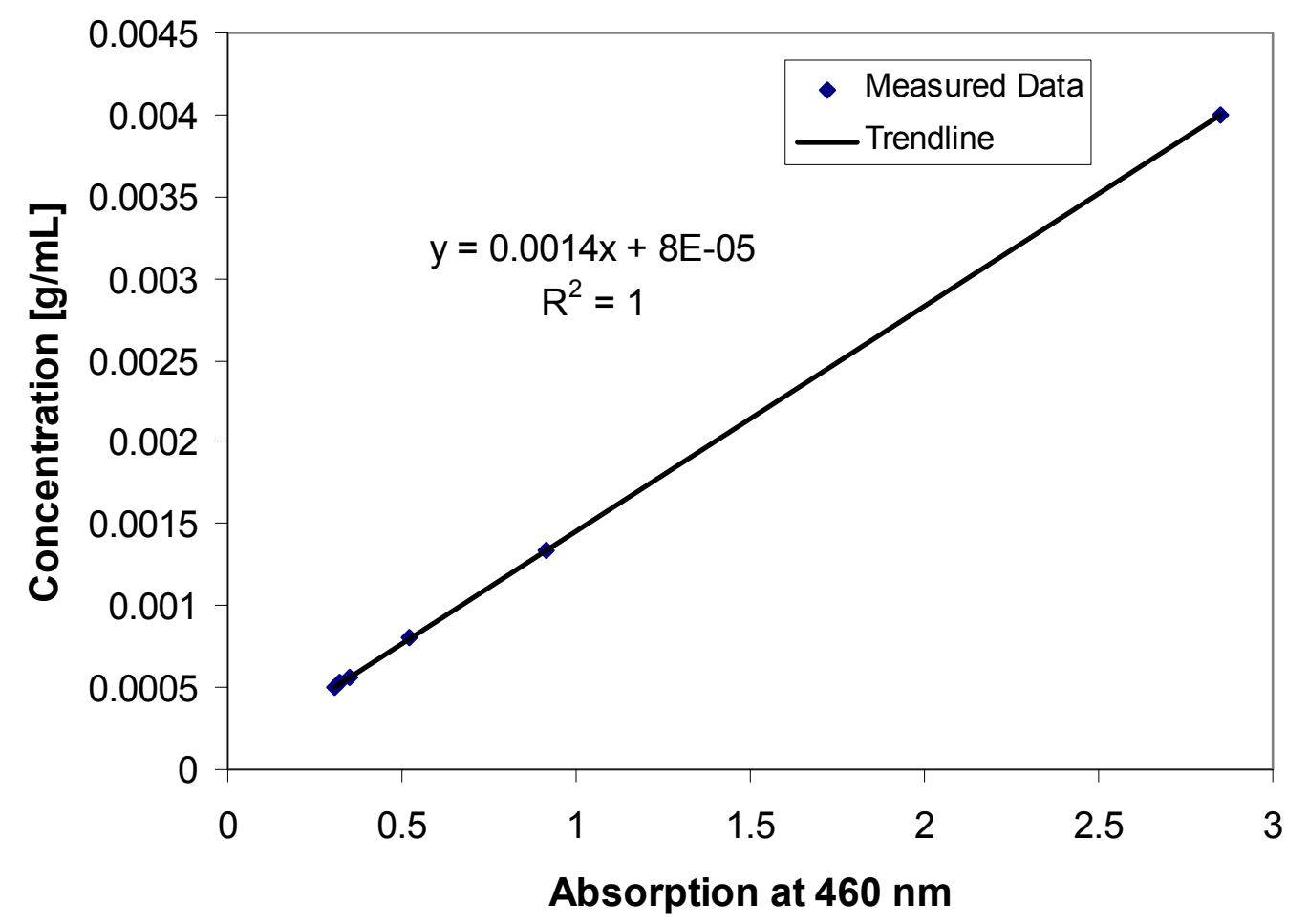

Figure 3-8: Correlation of concentration to absorbance for dilutions of iron oxide nanofluid with known concentrations. 
Using the correlation shown in Figure 3-8, the concentration of the trapped nanofluid was estimated to be $0.00057 \mathrm{~g} / \mathrm{ml}$. The collection efficiency was calculated using Equation 32 .

$$
\eta_{\text {collection }}=\frac{m_{\text {collected }}}{m_{\text {injected }}} * 100 \%=\frac{C_{\text {collected }} V_{\text {collected }}}{C_{\text {injected }} V_{\text {injected }}} * 100 \%
$$

where $\eta_{\text {collection }}$ is collection efficiency, $m$ denotes mass, $C$ denotes concentration, and $V$ denotes volume. The collection efficiency for this experiment was estimated to be $3 \%$. 


\section{Chapter 4}

\section{Analysis of Tracer Return Curves to Estimate Measurement Geolocation}

It has been shown that silica nanoparticles with covalently attached dye have potential to measure temperature in geothermal reservoirs (Alaskar et al., 2011). However, simply knowing that some region of the reservoir has a certain temperature without knowing the geolocation of the measurement is of limited use to reservoir engineers. If this geolocation could be estimated accurately, the reservoir temperature distribution could be mapped. This could make it possible to predict thermal breakthrough in a reservoir and would allow reservoir engineers to make more informed decisions. The potential capability of nanosensors with a dye-release sensing mechanism (e.g. dye-attached silica nanoparticles) to estimate measurement geolocation via analysis of the return curve of released dye is addressed in this chapter.

\subsection{Simple Analytical Model for Return Curve Analysis}

A thought experiment was performed consisting of two hypothetical tracer tests performed in a well doublet: one with a conservative solute tracer and one with dyereleasing nanosensors. Synthetic tracer return curves for these hypothetical tests were generated using an analytical solution to the advection-dispersion equation, which is often used to describe subsurface tracer flow. Juliusson and Horne (2011) expressed the one-dimensional form of this equation as:

$$
R \frac{\partial C}{\partial t}=\alpha u \frac{\partial^{2} C}{\partial x^{2}}-u \frac{\partial C}{\partial x}
$$

where $C$ is tracer concentration, $x$ is the spatial coordinate, $t$ is time, $u$ is the flux velocity, and $\alpha$ is the dispersion length, and $R$ is the tracer retardation factor, which accounts for the retardation of tracer transport caused by the reversible adsorption of tracer to rock interfaces. For this initial investigation, the simple case with a constant flux velocity $v$ (i.e. constant flow rate) was considered, and it was assumed that $R$ is constant with respect to $t, x$, and $C$ for both the solute tracer and the nanosensors. It may be more realistic to assume that $R$ varies spatially, as is suggested by Chrysikopoulos (1993), and that nanoparticle flow likely requires even more complex treatment. With these caveats in mind, it was decided to first examine the simplest possible case.

Kreft and Zuber (1978) provided a solution to the advection-dispersion equation with flux injection and detection boundary conditions, and Juliusson and Horne (2011) rewrote this solution to include the retardation factor $R$ : 


$$
\begin{gathered}
C=\frac{m V_{x}}{q t \sqrt{4 \pi V_{\alpha} q t}} \exp \left(-\frac{\left(q t-V_{x}\right)^{2}}{4 V_{\alpha} q t}\right) \\
V_{x}=R A \phi x \\
V_{\alpha}=R A \phi \alpha
\end{gathered}
$$

where $m$ is the mass injected, $q$ is the volumetric flowrate, $t$ is time, $V_{x}$ is the pore volume modified by the retardation factor $R, V_{\alpha}$ is the dispersion volume modified by $R, A$ is cross-sectional area, $\varphi$ is porosity, and $\alpha$ is the dispersion length.

Consider a flow-path in a geothermal reservoir between a well doublet that consists of a single fracture with length $L$, cross-sectional area $A$, and porosity $\varphi$. Before extraction, the reservoir had a uniform temperature distribution with a temperature of $T_{1}$. Some years after the onset of extraction, the thermal front has advanced to the position $x_{f}$, and the portion of the reservoir behind the front has cooled to temperature $T_{2}$, as shown in Figure 4-1. Suppose a nanosensor has been designed to release a fluorescent dye at the threshold temperature $T_{1}$, and assume that this release occurs instantaneously upon exposure to this threshold.

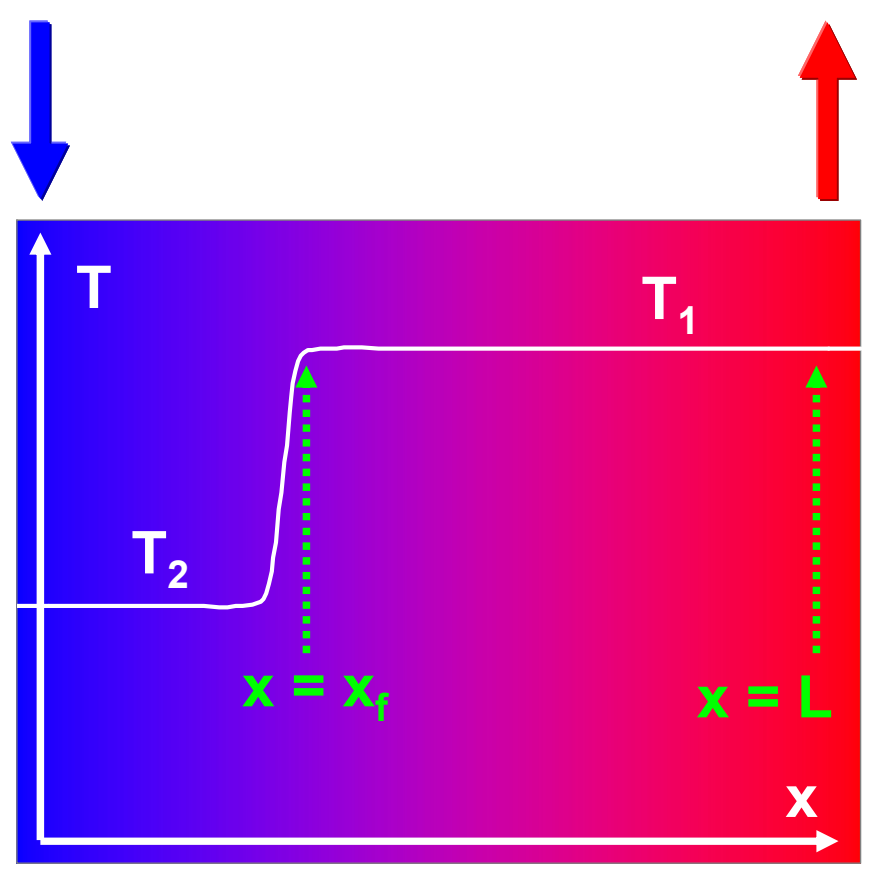

Figure 4-1: Cartoon of temperature distribution in a geothermal reservoir with a thermal front at position $\mathrm{x}_{\mathrm{f}}$.

Two tracer tests are performed. In one test, a mass $m_{c}$ of a conservative tracer with a retardation factor $R_{c}$ is injected into the reservoir. The tracer is sampled at the production well (i.e. $x=L$ ), and the return curve can be described by Equations 4-5 through 4-7: 


$$
\begin{gathered}
C_{c}=\frac{m_{c} V_{x, c}}{q t \sqrt{4 \pi V_{\alpha, c} q t}} \exp \left(-\frac{\left(q t-V_{x, c}\right)^{2}}{4 V_{\alpha, c} q t}\right) \\
V_{x, c}=R_{c} A \phi L \\
V_{\alpha, c}=R_{c} A \phi \alpha
\end{gathered}
$$

where the subscript $c$ denotes the conservative solute tracer.

In the second test, a slug of the dye-releasing nanosensors with retardation factor $R_{n}$ is injected into the reservoir. Upon reaching exposure to the threshold temperature $T_{1}$ at position $x_{f}$, the nanosensors release a mass $m_{r}$ of the attached dye, which itself behaves like the conservative solute tracer in the previous tracer test, and has a retardation factor $R_{c}$. The released dye is sampled at the production well. The return curve of the released dye is influenced by both $R_{n}$ and $R_{c}$, because it travels with the nanosensor retardation factor $R_{n}$ from $x=0$ to $x=x_{f}$ and the retardation factor $R_{c}$ from $x=x_{f}$ to $x=L$. Thus, the return curve of the released dye can be described by convolution, as shown in Equations 4-8 through 4-11:

$$
\begin{gathered}
C_{r}=\int_{0}^{t} \frac{m_{r} V_{x, n}}{q t \sqrt{4 \pi V_{\alpha, n} q(t-\tau)}} \exp \left(-\frac{\left(q(t-\tau)-V_{x, n}\right)^{2}}{4 V_{\alpha, n} q(t-\tau)}\right) \cdot \frac{q V_{x, r}}{q t \sqrt{4 \pi V_{\alpha, c} q \tau}} \exp \left(-\frac{\left(q \tau-V_{x, r}\right)^{2}}{4 V_{\alpha, c} q \tau}\right) \partial \tau \\
V_{x, n}=R_{n} A \phi x_{f} \\
V_{\alpha, n}=R_{n} A \phi \alpha \\
V_{x, r}=R_{c} A \phi\left(L-x_{f}\right)
\end{gathered}
$$

where the subscript $n$ denotes the nanosensors and the subscript $r$ denotes the released dye. Note that $V_{x, r}$ can be rewritten as:

$$
V_{x, r}=V_{x, c}-\frac{V_{\alpha, c}}{V_{\alpha, n}} V_{x, n}
$$

Thus, the return curves for the conservative tracer can be fit to Equation 4-5 by adjusting the values of unknowns $V_{x, c}$ and $V_{\alpha, c}$ (i.e. minimizing the norm of the differences between the return curve data and the model with the unknowns as decision variables). Subsequently, the return curves for the released dye can be fit to Equation 4-8 by adjusting the values unknowns $V_{x, n}$ and $V_{a, n}$. Note that $V_{x, r}$ is not explicitly adjusted here because it can be expressed in terms of the other unknowns. Once the values of the unknowns have been determined, one can calculate the geolocation of the thermal front using Equation 4-13:

$$
x_{f, d}=\frac{x_{f}}{L}=\frac{V_{x, c}-V_{x, r}}{V_{x, c}}
$$

where $x_{f, d}$ is the dimensionless position of the thermal front. 


\subsection{Example Problem}

This analysis was demonstrated successfully in an example problem with the parameter values shown in Table 4-1.

Table 4-1: Parameter Values Used In Return Curve Analysis Demonstration Problem

\begin{tabular}{|l|l|}
\hline Parameter & Value \\
\hline$R_{c}$ & 2 \\
\hline$R_{n}$ & 1 \\
\hline$A$ & $200 \mathrm{~m}^{2}$ \\
\hline$\varphi$ & 0.10 \\
\hline$L$ & $1000 \mathrm{~m}$ \\
\hline$\alpha$ & $25 \mathrm{~m}$ \\
\hline$P e=L / \alpha$ & 40 \\
\hline$m_{c}$ & $123.75 \mathrm{~kg}$ \\
\hline$m_{r}$ & $123.75 \mathrm{~kg}$ \\
\hline$q$ & $43.2 \mathrm{~m}^{3} / \mathrm{hr}$ \\
\hline
\end{tabular}

These values were chosen somewhat arbitrarily for the purposes of this investigation. However, values of $R_{c}$ and $R_{n}$ were used such that the nanosensors experience no retardation and the solute tracer does experience retardation. This is based on studies of colloid transport in fractures which showed that non-adsorbing colloids exhibit breakthrough more rapidly than solute tracers because they tend to stay in fluid streamlines and do not experience matrix diffusion (Reimus 1995). Also, the volumetric flow rate $q$ and the masses of the tracer slugs $m_{c}$ and $m_{r}$ were based on values reported by Gentier et al. (2010) for the 2005 tracer test performed in the Soultz-sous-Forets reservoir.

Synthetic return curve data for the conservative tracer and the nanosensors were generated for various values of $x_{f}$ using Equations 4-5 and 4-8, and Gaussian noise was added for the sake of realism. An optimization solver was then used to find the best fit to Equations 4-5 and 4-8 by adjusting the unknowns. The results are tabulated in Table 4-2, and select return curves are plotted in Figure 4-2.

Table 4-2: Estimates of Temperature Measurement Geolocations In Demonstration Problem For Various True Values of $x_{f, d}$

\begin{tabular}{|c|c|c|}
\hline $\mathbf{x}_{\mathbf{f}, \mathbf{d}}$ true & $\mathbf{x}_{\mathbf{f}, \mathbf{d}}$ estimate & Error \\
\hline 0.05 & 0.037 & $26 \%$ \\
\hline 0.15 & 0.155 & $3.2 \%$ \\
\hline 0.25 & 0.248 & $0.8 \%$ \\
\hline 0.35 & 0.382 & $9.1 \%$ \\
\hline 0.45 & 0.431 & $4.3 \%$ \\
\hline 0.55 & 0.517 & $5.9 \%$ \\
\hline 0.65 & 0.632 & $2.7 \%$ \\
\hline 0.75 & 0.746 & $0.6 \%$ \\
\hline
\end{tabular}




\begin{tabular}{|l|l|l|}
\hline 0.85 & 0.852 & $0.2 \%$ \\
\hline 0.95 & 0.925 & $2.7 \%$ \\
\hline
\end{tabular}
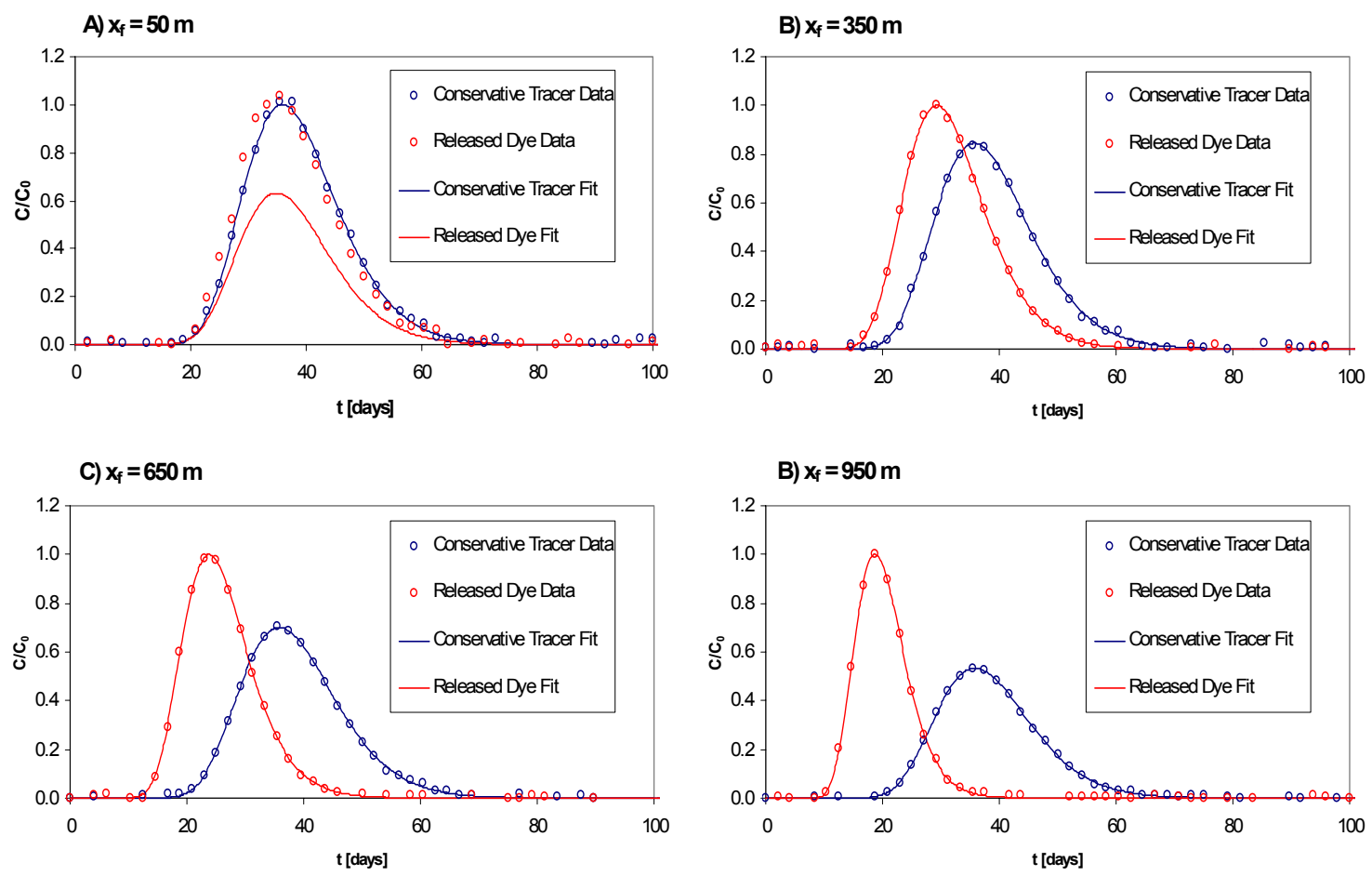

Figure 4-2: Return curve data and fits for A) $\left.\left.\mathrm{x}_{\mathrm{f}}=50 \mathrm{~m}, \mathrm{~B}\right) \mathrm{x}_{\mathrm{f}}=350 \mathrm{~m}, \mathrm{C}\right) \mathrm{x}_{\mathrm{f}}=650 \mathrm{~m}$, and D) $x_{\mathrm{f}}=950 \mathrm{~m}$. Note that released dye experiences breakthrough first because it is carried a distance $\mathrm{x}_{\mathrm{f}}$ by the nanosensor, which has a retardation factor of 1 , while the conservative tracer has a retardation factor of 2 .

Reasonably good estimates of the geolocation of the thermal front were obtained for all scenarios except for $x_{f}=0.05$. This is physically intuitive, because the return curves for the conservative tracer and the released dye are almost identical when the thermal front is still close to the injection well, making it difficult to estimate the front geolocation quantitatively. The poor fit of the return curve of the released dye can be attributed mathematically to the problematic nature of the optimization surface for this scenario, which is shown in Figures 4-3 and 4-4. 


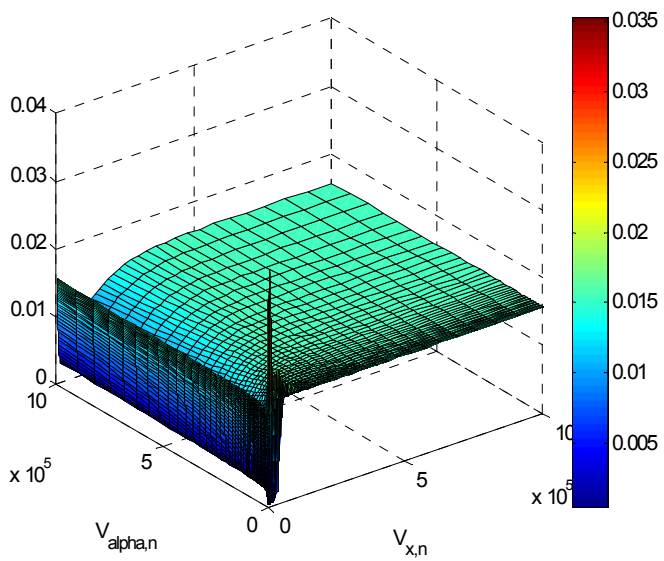

Figure 4-3: Objective function surface for fitting the return curve of the reactive tracer when $\mathrm{x}_{\mathrm{f}}=50 \mathrm{~m}$.

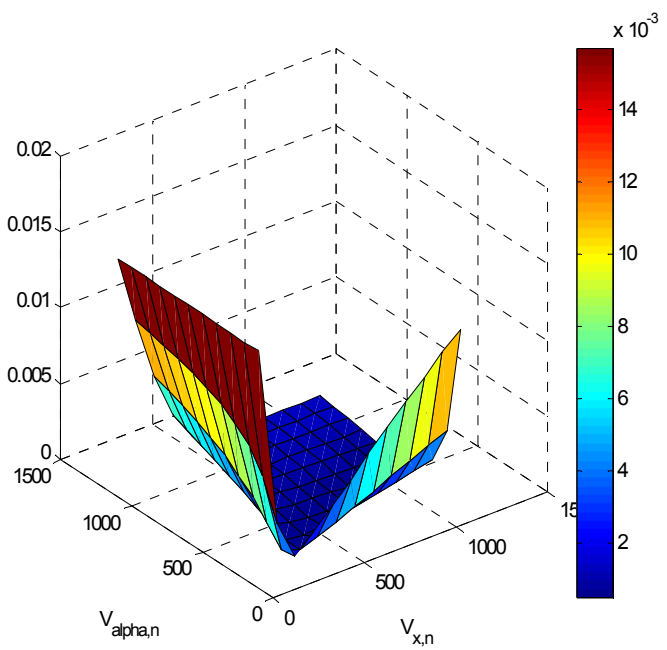

Figure 4-4: Objective function surface for fitting the return curve of the reactive tracer when $\mathrm{x}_{\mathrm{f}}=50 \mathrm{~m}$, zoomed in near the minimum of $\left(\mathrm{V}_{\mathrm{x}, \mathrm{n}}=1000 \mathrm{~m}^{3}, \mathrm{~V}_{\alpha, \mathrm{n}}=500 \mathrm{~m}^{3}\right)$. Note that the point chosen by the solver was $\left(\mathrm{V}_{\mathrm{x}, \mathrm{n}}=268.3 \mathrm{~m}^{3}, \mathrm{~V}_{\alpha, \mathrm{n}}=180.8 \mathrm{~m}^{3}\right)$.

The large trough along the $V_{\alpha, n}$ axis in Figure 4-3 indicates that for large initial guesses of $V_{\alpha, n}$, the solver might get stuck far from the minimum (since change in the objective function is the termination criteria). Moreover, the values of the objective function vary very little near the minimum, as shown in Figure 4-4. This explains why the solver terminated at the point $\left(V_{x, n}=268.3 \mathrm{~m}^{3}, V_{\alpha, n}=180.8 \mathrm{~m}^{3}\right)$ and resulted in a poor fit. 


\section{Chapter 5}

\section{Tin-bismuth Alloy Nanosensors}

Tin-bismuth alloy nanoparticles were chosen to investigate temperature-sensitive nanosensors with a melting sensing mechanism. This alloy has a melting temperature that is tunable between $139^{\circ} \mathrm{C}$ and $271^{\circ} \mathrm{C}$ by adjusting its composition, as shown in the phase diagram in Figure 5-1. A simple sensing scheme of melting and subsequent particle growth was conceived. The growth of native gold nanoparticles upon melting in geologic Arsenian pyrite has been observed by Reich et al. (2006) using transmission electron microscopy during in situ heating to $650^{\circ} \mathrm{C}$. It was shown that when the gold nanoparticles melted, they became unstable, leading to diffusion-driven Ostwald ripening and ultimately resulting in the coarsening of the size distribution. We hypothesized that upon melting, the size distribution of tin-bismuth alloy nanoparticles would also coarsen.

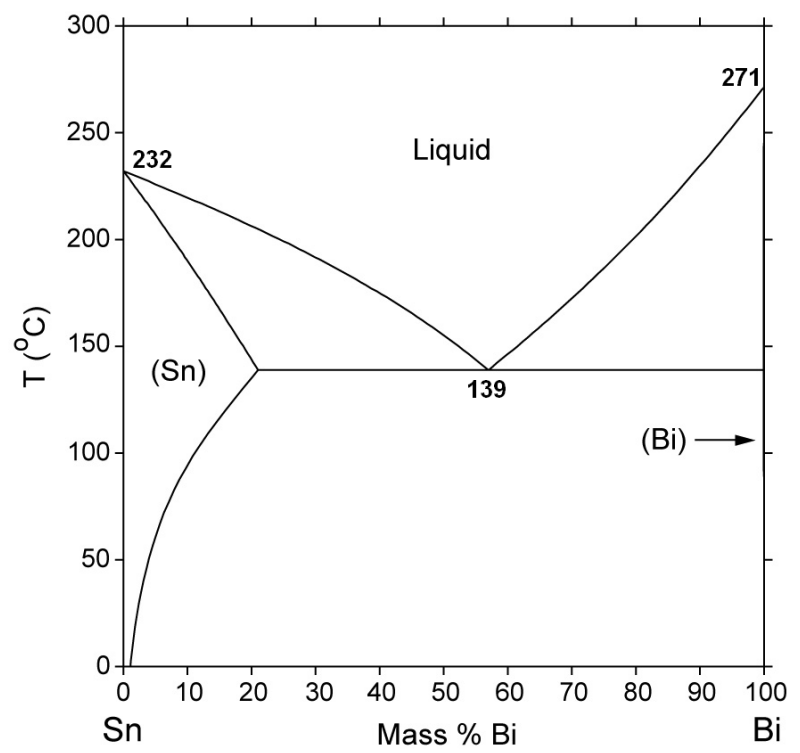

Figure 5-1: Phase diagram of tin-bismuth. Reproduced from National Institute of Standards and Technology.

Tin-bismuth alloy nanoparticles of eutectic composition were synthesized via ultrasonic processing. These particles were characterized using DLS and SEM imaging. A bench heating experiment was also performed to study the thermal sensitivity of these nanoparticles. The sample was characterized again with DLS and SEM imaging after heating. Mohammed Alaskar performed injection experiments in a slim-tube packed with glass beads and a Berea sandstone core and analyzed effluent samples with DLS and SEM imaging. 


\subsection{Synthesis of tin-bismuth alloy nanoparticles}

To perform the synthesis, tin and bismuth were melted together at the eutectic composition ( $\sim 60 \mathrm{wt} \%$ bismuth and $\sim 40 \mathrm{wt} \%$ tin). After the alloy was cooled to room temperature, $100 \mathrm{mg}$ was sonicated in $10 \mathrm{ml}$ of mineral oil, a slight variation of the sonochemical method suggested by Chen et al. (2005). The VC-505 ultrasonic processor manufactured by Sonics \& Materials, Inc. with a 0.75 in. diameter high gain solid probe was used. The sonicator was operated at $200 \mathrm{~W}$ ( $\sim 95 \%$ amplitude) with a pulse setting of $20 \mathrm{~s}$ on, $10 \mathrm{~s}$ off. The mixture was cooled to room temperature. The alloy particles were washed and centrifuged several times with a 1:1 mixture of hexane and acetone, rinsed in a solution of $0.1 \mathrm{M}$ polyvinyl pyrrolidone (PVP) in ethanol, and finally suspended in ethanol. The centrifuge setting was $6000 \mathrm{rpm}$ for 15 minutes each time.

\subsection{Characterization of tin-bismuth alloy nanoparticles}

The tin-bismuth alloy nanoparticles were characterized in terms of size and shape using DLS and SEM imaging.

It was determined from three consecutive DLS measurements that there was a wide distribution of the particle hydrodynamic diameter, as shown in Figure 5-2.

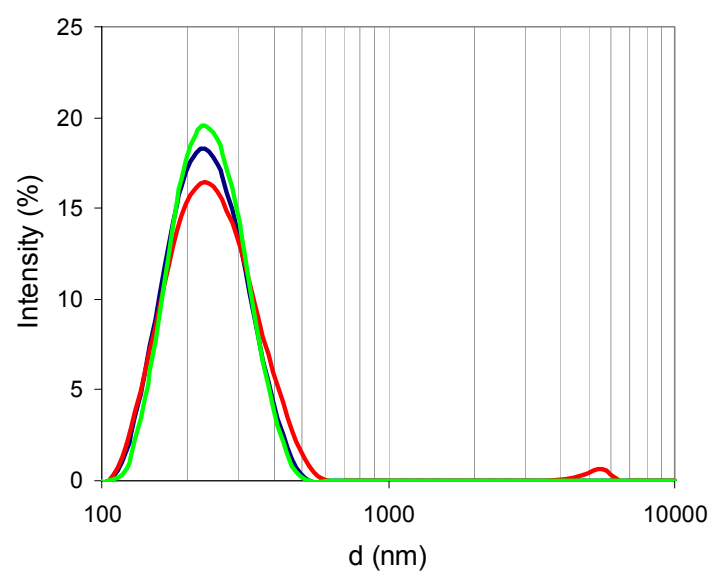

Figure 5-2: Logarithmic particle size distribution based on hydrodynamic diameter for original tin-bismuth nanoparticle sample.

The three measurements are in reasonable agreement, with an average modal value of $235 \mathrm{~nm}$. The hydrodynamic diameter ranged from $\sim 100 \mathrm{~nm}$ to $\sim 600 \mathrm{~nm}$, with Run 2 showing a small peak at $\sim 5500 \mathrm{~nm}$. This indicates that there may have been large particles in the sample, either due to aggregation or from the original synthesis.

The SEM images of the sample show good agreement with the DLS measurements, as shown in Figures 5-3. 

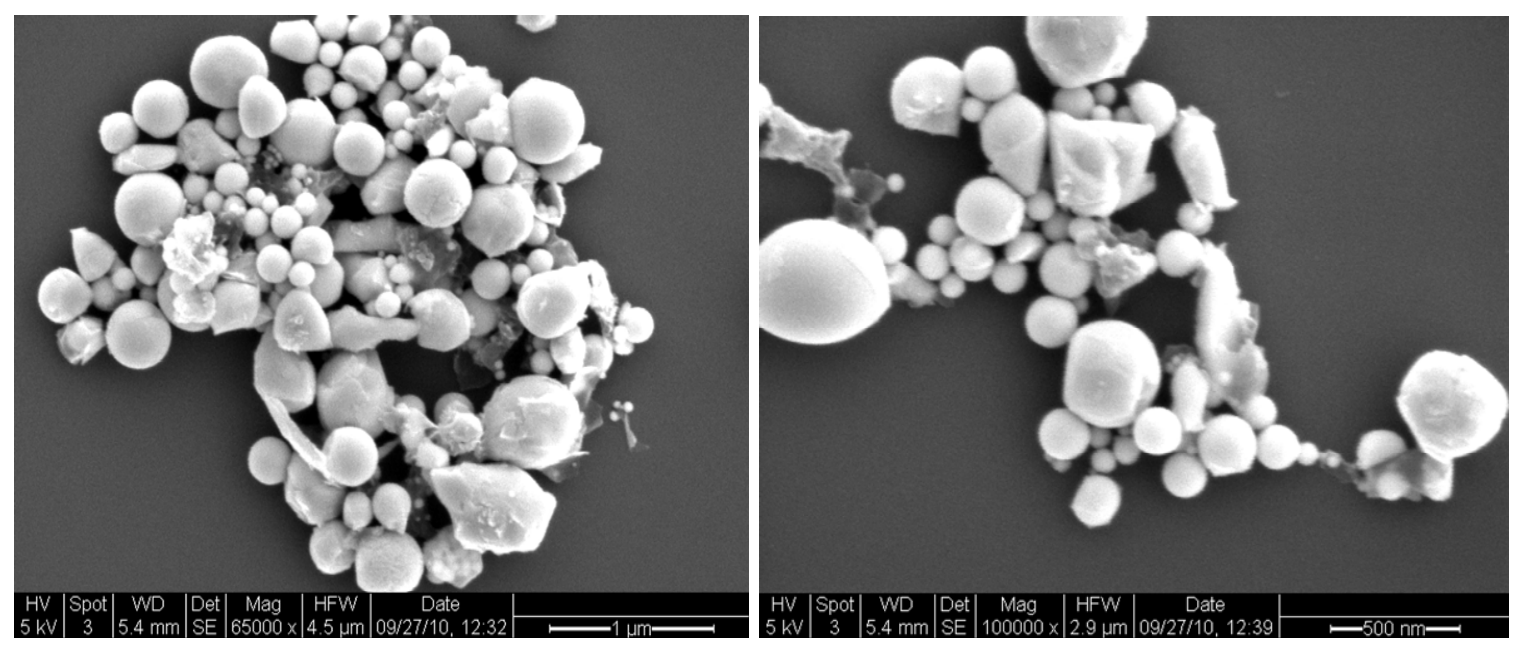

Figure 5-3: SEM images of tin-bismuth nanoparticles at higher magnification.

It is apparent from Figure 5-3 that the tin-bismuth nanoparticles range from $50 \mathrm{~nm}$ to less than $600 \mathrm{~nm}$. Furthermore, although many of the nanoparticles seem to be spherical as expected, the presence of nonspherical crystalline structures indicates that the sonochemical synthesis did not reach completion. Aggregation on the substrate is observed in both figures, but it is unclear whether this aggregation occurs in solution or upon drying on the substrate. The DLS results suggest that the latter may be the case.

\subsection{Tin-bismuth nanoparticle heating experiment}

To begin investigating the melting behavior of tin-bismuth nanoparticles within the temperature range of interest, a sample of tin-bismuth nanoparticles in mineral oil (i.e. a sample taken before washing and suspending in ethanol) was subjected to a preliminary heating experiment. Although ultimately we are interested in the melting behavior of the tin-bismuth nanoparticles in water, the heating experiments were performed in oil due to the complications associated with the boiling of water at experimental conditions. As shown in the phase diagram Figure 5-1, at the eutectic composition, the tin-bismuth alloy melts at $139^{\circ} \mathrm{C}$. In fact, the nanoparticles likely melt at a slightly lower temperature than this because of melting point depression due to their size.

The sample was heated using a heating mantle connected to a temperature controller with a feedback thermometer, as shown in Figure 5-4. The temperature controller was also connected to a thermometer, the feedback from which affected whether the mantle was heated, cooled, or maintained and the rate at which this was done. The thermometer was positioned in port A. 


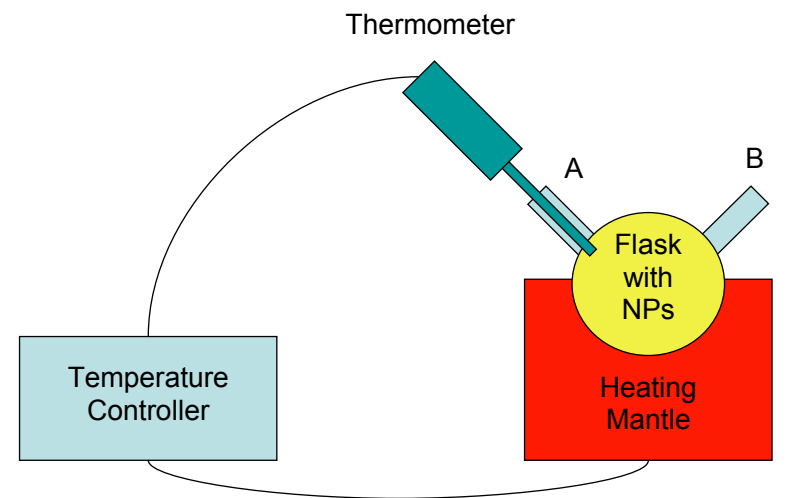

Figure 5-4: Experimental apparatus for tin-bismuth heating experiment

The sample was heated in steps to the expected melting point of $139^{\circ} \mathrm{C}$. The sample was monitored for a color change near the expected melting point, and when none occurred, the sample was heated in steps to $210^{\circ} \mathrm{C}$. No color change ever occurred, but the heating was stopped to prevent the mineral oil from burning. It was hypothesized that melting occurred despite the absence of color change. Finally, when the apparatus was at room temperature, the sample was removed from the flask.

The sample was then washed and centrifuged several times with a 1:1 mixture of hexane and acetone, rinsed in a solution of $0.1 \mathrm{M}$ PVP in ethanol, and finally suspended in ethanol. The centrifuge setting was $6000 \mathrm{rpm}$ for 15 minutes each time. This sample was characterized using DLS and SEM imaging.

The DLS results of the sample subjected to heating are shown in Figure 5-5.

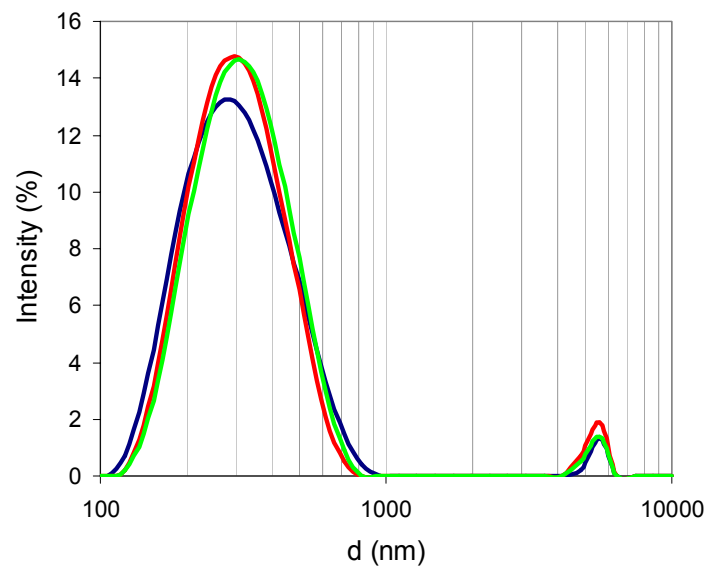

Figure 5-5: Logarithmic particle size distribution based on hydrodynamic diameter for heated tinbismuth nanoparticle sample.

The three measurements are in relatively close agreement, with an average modal value of $321 \mathrm{~nm}$. The hydrodynamic diameter ranged from $\sim 100 \mathrm{~nm}$ to $\sim 1000 \mathrm{~nm}$. Appreciable secondary peaks in the range of $\sim 4100 \mathrm{~nm}$ to $\sim 6400 \mathrm{~nm}$ were observed for all runs. This indicates that there are large particles in the sample, most likely due to aggregation and 
fusion of the particles. A comparison of the particle size distribution curves of the original and heated samples is shown in Figure 5-6. As shown in the Figure 5-6, the particle size distribution peak shifted noticeably to a larger size. Also, the secondary peak in the micron scale is noticeably larger, indicating that there may be more large aggregates. SEM images of the heated sample are shown in Figures 5-7.

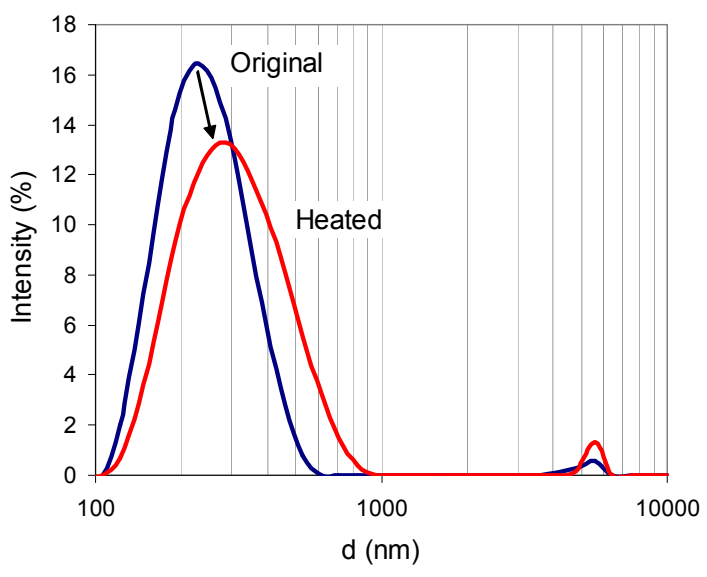

Figure 5-6: Comparison of logarithmic particle size distribution based on hydrodynamic diameter for original and heated tin-bismuth nanoparticle samples.
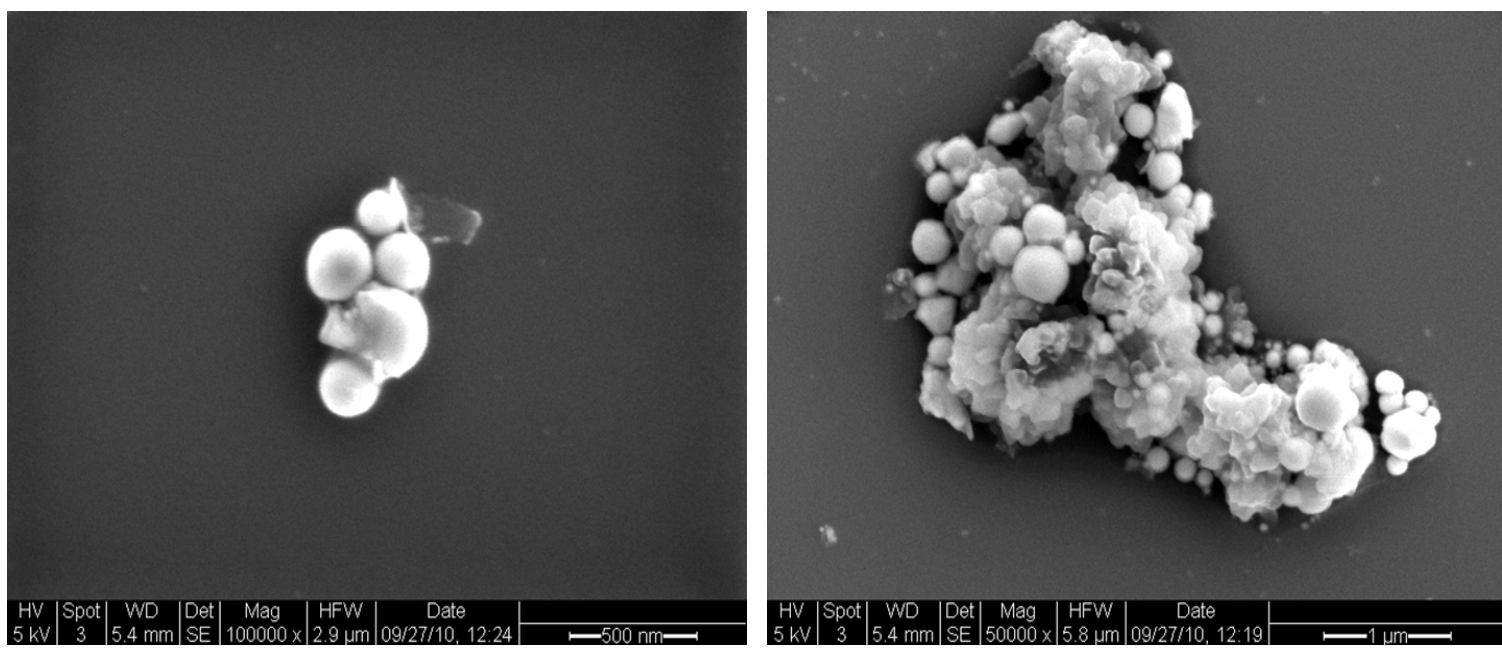

Figure 5-7: SEM images showing heated tin-bismuth nanoparticles.

Fusion of melted particles can be observed in Figure 5-7, and the sizes of both particles and large aggregates are within the range suggested by DLS results. While the fusion of melted particles could account for the shift in particle size distribution, it is difficult to reach any definite conclusions from the SEM results due to the very wide particle size distributions of both the heated and unheated samples. To avoid this ambiguity, a new synthesis will be attempted in order to achieve a more uniform particle size distribution. Additionally, heating experiments will be repeated directly on a silicon substrate, and a small region of this substrate will be marked for unambiguous SEM analysis. 


\subsection{Tin-Bismuth Nanoparticle Injection Experiments}

Two injection experiments were carried out by Mohammed Alaskar to investigate the mobility of tin-bismuth nanoparticles in porous media: one in a Berea sandstone core, and one in a slim-tube packed with glass beads. The results of these experiments are summarized here because of their relevance to the project as a whole.

The injection into the Berea core had interesting results. As soon as the nanofluid was injected, there was a sudden drop in permeability to $56 \%$ of its original value. With continued injection, the permeability increased until it reached a plateau at $82 \%$ of its original value. Finally, after injection was completed, the core was back-flushed, resulting in a permeability improvement to $90 \%$ of its original value. These changes in permeability are plotted in Figure 5-8.

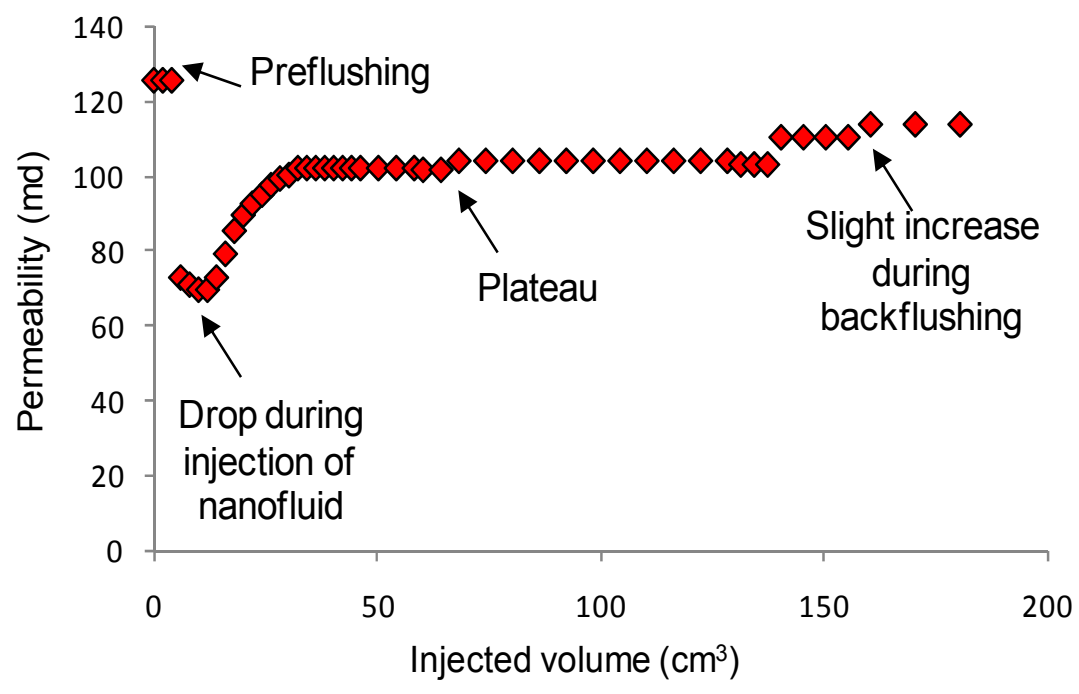

Figure 5-8: Permeability measurements during injection of tin-bismuth nanoparticles into Berea sandstone core. Reproduced from Alaskar et al. (2011).

In an attempt to explain these permeability changes, effluent samples from the plateau region were characterized using SEM. As shown in Figure 5-9, tin-bismuth nanoparticles were present in this effluent sample. However, the sample was dilute and only particles with diameters of $200 \mathrm{~nm}$ and smaller were found. This means that the larger particles were trapped somewhere within the core. Next, samples from the back-flushing were characterized using SEM imaging. As shown in Figure 5-9, particles with diameters larger than $450 \mathrm{~nm}$ were back-flushed from the core, indicating that nanoparticles were trapped at the core inlet. 

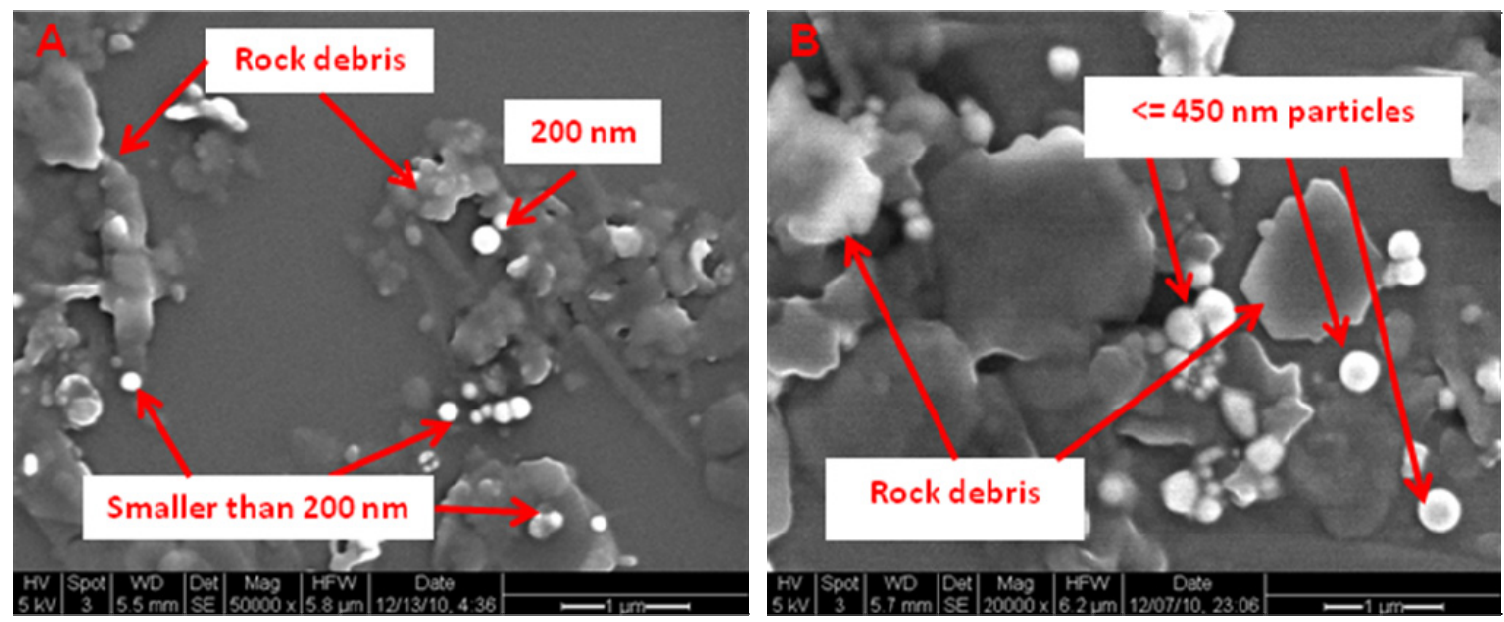

Figure 5-9: SEM images showing tin-bismuth nanoparticles in the effluent from (a) injection experiment and (b) backflushing experiment. Reproduced from Alaskar et al. (2011).

A thin slice was taken at the core inlet and analyzed using SEM, as shown in Figure 5-10. This confirmed that most of the tin-bismuth nanoparticles were trapped at the core inlet.
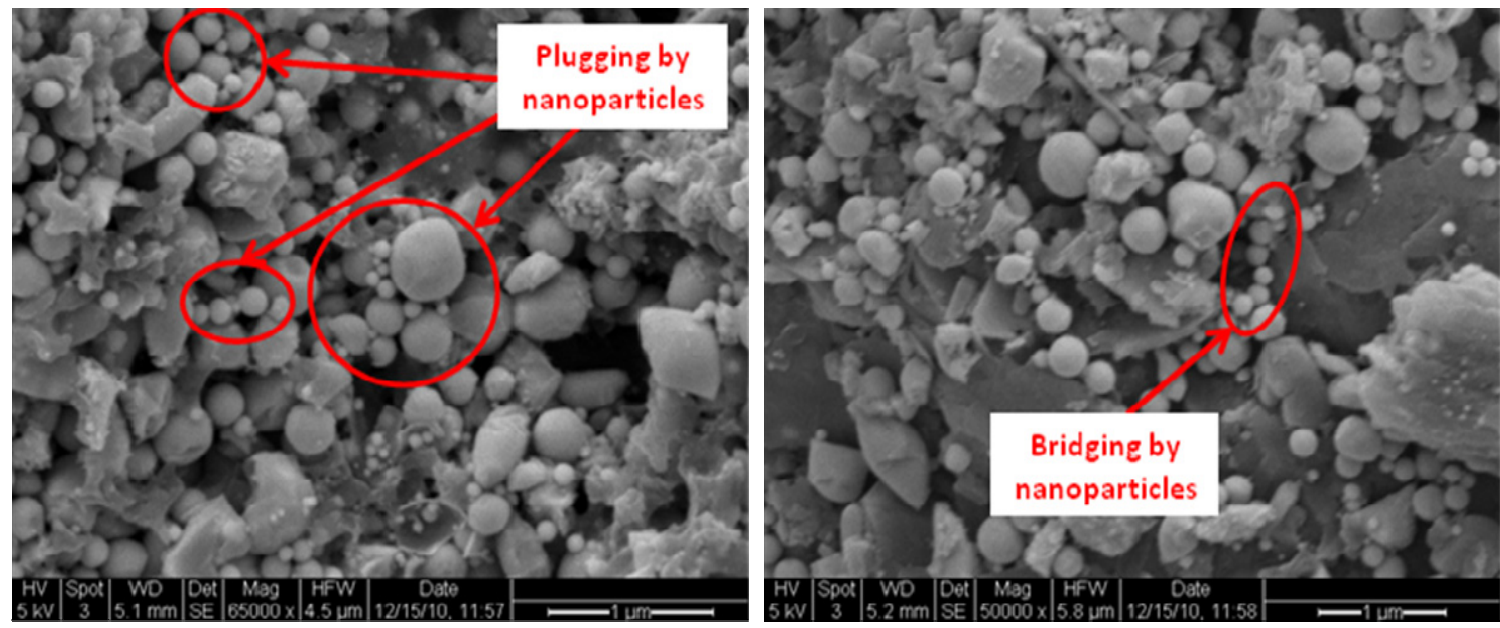

Figure 5-10: SEM images of the inlet to the Berea sandstone core with evidence of nanoparticle trapping. Reproduced from Alaskar et al. (2011).

This raised the question as to why the recovery was poor. Kanj et al (2009) suggest that nanoparticle mobility in rock can be limited by shape and surface charge, influent concentration, particle settling, affinity to the rock itself. Silica nanoparticles of the same shape and similar surface charge were previously transported through a Berea core, indicating that these are not the factors limiting flow (Alaskar et al., 2010).

To further investigate the role of rock interactions, tin-bismuth nanoparticles were injected into a slim tube packed with glass beads. The objective of this injection was to test the mobility of tin-bismuth nanoparticles in the absence of rock material. A sample of the same concentration as that in the Berea injection was used. Tin-bismuth nanoparticles were concentrated enough in the effluent to be observed visually, as shown in Figure 5- 
11. SEM analysis showed that all particle sizes were present in the effluent, as shown in Figure 5-12. This indicated that particle mobility in the sandstone core may have been limited by pore complexity or a possible affinity of the particles to the rock.

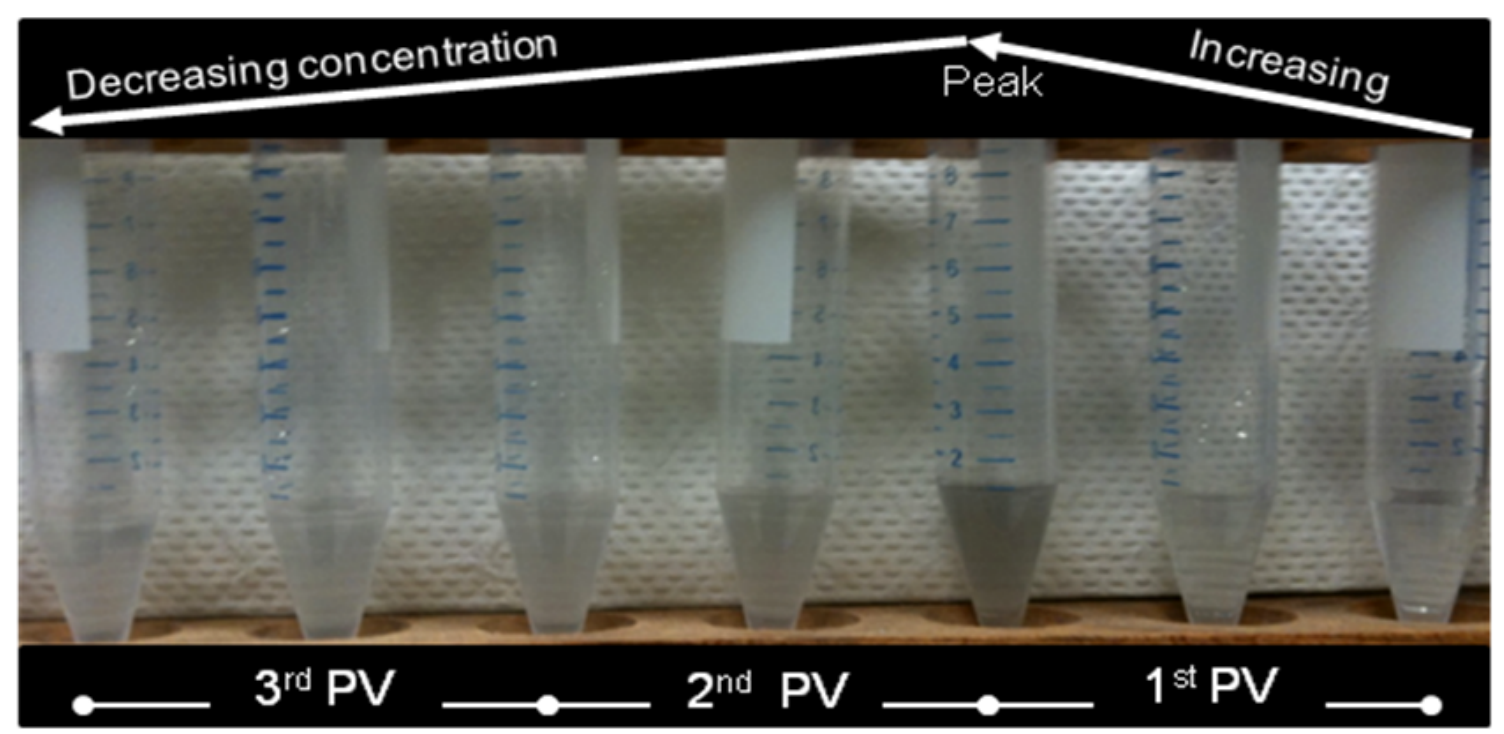

Figure 5-11: Visual observation of tin-bismuth nanoparticles in the effluent samples from the slim-tube injection experiment. Reproduced from Alaskar et al. (2011).
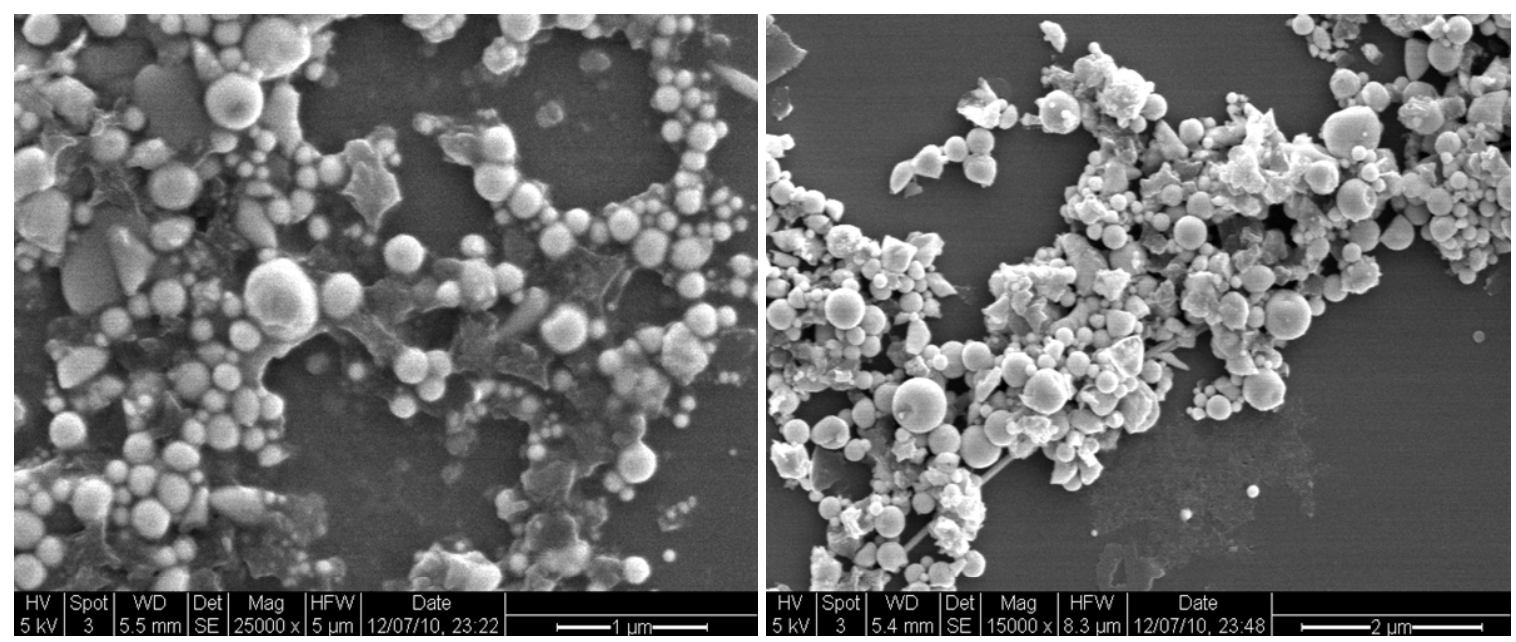

Figure 5-12: SEM images showing tin-bismuth nanoparticles of all sizes in the effluent from the slim-tube injection experiment. Reproduced from Alaskar et al. (2011). 


\section{Chapter 6}

\section{Conclusions and Future Work}

The development of geothermal energy resources, both conventional hydrothermal and EGS, could be made more economical if the temperature distribution of the reservoir could be measured. The recoverable energy in place is directly related to the temperature distribution, and having this information could lead to more accurate forecasting of reservoir life and facilitate more informed reservoir management decisions. However, the reservoir temperature can currently only be measured at or very near the wellbore. Many have proposed that reactive solute tracers can be used to measure temperature distribution and predict thermal breakthrough, but this has yet to be successfully demonstrated in actual practice.

As reservoir engineering tools, nanosensors have significant potential to measure temperature distribution because of a high degree of control over their physical and chemical properties. There are significant technical challenges associated with the development of nanosensors for geothermal applications: mobility in the reservoir, the capability to collect and detect them in low concentrations in produced fluid, the requirement of a predictable and irreversible temperature sensing mechanism, and inferring the location of temperature measurement in order to spatially map temperature distribution. Several candidate sensors have been identified for investigation: melting tinbismuth alloy nanoparticles, silica nanoparticles with covalently attached fluorescent dye, hollow silica nanoparticles with encapsulated fluorescent dye and an impermeable, melting shell, and dye-polymer composite time-temperature indicators.

In order to investigate the mobility of silica nanoparticles in long flow paths in porous media, an injection experiment was performed using a $10 \mathrm{~m}$ long slim-tube packed with sand. Nanoparticles were observed visually in the effluent, and their presence was confirmed by DLS and SEM characterization. A backflushing experiment was also performed, and some nanoparticles were also detected in the effluent samples from this experiment, indicating that some particles had been trapped. Nevertheless, the proof of concept that silica nanoparticles can be transported through long flow paths in porous media was established.

To investigate the potential to collect nanoparticles from produced fluid magnetically, a magnetic collection experiment was performed using paramagnetic iron oxide nanoparticles and neodymium permanent magnets. Most of the nanoparticles were observed visually passing through the magnetic trap without appearing to be collected, but a small amount of nanoparticles remained trapped at the end of the experiment. The concentration of the collected nanofluid was calculated from absorbance, which was 
measured using uv vis spectroscopy. This concentration was used to estimate that $3 \%$ of the injected particles were collected at the magnet. While this is a low collection efficiency, this experiment showed that there is potential to collect nanosensors in this manner. The most promising way to increase the effectiveness of such a collection scheme is to increase the applied magnetic field, as particle size and composition are constrained by mobility concerns.

To emphasize the potential usefulness of a nanosensor with a dye-release mechanism (such as dye-attached silica nanoparticles), an initial study was performed to evaluate the feasibility of estimating the geolocation of temperature measurements by analyzing return curves of released dye. Accurate estimation of measurement geolocations would allow mapping of temperature distribution in a geothermal reservoir and provide information about thermal breakthrough. A simplified example problem was examined in which an analytical solution of the one-dimensional advection-dispersion equation was used to generate synthetic return curve data for two tracer tests: one with a conservative tracer and one with dye-releasing nanosensors. Gaussian noise was then added to the synthetic data. Finally, return curves were fit to the data by adjusting the values of four unknowns (two unknowns for each return curve). Reasonably accurate estimates of the measurement geolocation were achieved for various scenarios, with the exception of a poor estimate when the thermal front had not advanced very far into the reservoir.

Initial investigations were performed to evaluate the suitability of tin-bismuth alloy nanoparticles as nanosensors with a sensing mechanism of size increase induced by melting. Tin-bismuth was chosen as a candidate for investigation because its melting point can be tuned across a wide range of temperatures that are suitable for sensing in geothermal applications. Tin-bismuth alloy nanoparticles were synthesized using a sonochemical method. A heating experiment was performed using these nanoparticles, and particle growth was observed, which was indicative of melting. Injection experiments carried out by Mohammed Alaskar showed some challenges associated with tin-bismuth nanoparticle mobility in porous media, which may be due to an affinity of the nanoparticles to rock, gravitational settling, excessively high nanoparticle concentration in the influent, or filtration of large particles by size exclusion.

Future efforts will be devoted to each aspect of this complex problem. Each candidate sensor will be evaluated using bench heating experiments and injection experiments. Once a candidate is demonstrated to have good mobility and to undergo a predictable, detectable, and irreversible change upon exposure to a threshold temperature, a combined heat-and-flow experiment will be performed using the $25 \mathrm{~m}$ stainless steel slim-tube in an air bath. In the case of tin-bismuth nanoparticles, a new synthesis method will be used to obtain a more monodisperse sample with smaller particles, which will remove ambiguity about what limits its mobility. This will also remove ambiguity associated with the size change sensing mechanism. Magnetic collection experiments will be carried out with various collection schemes in order to find a configuration that maximizes collection efficiency. Finally, reservoir engineering techniques will be tailored to promising nanosensors in order to estimate measurement geolocation. In the case of dye-releasing nanosensors, more realistic and complex models will be investigated, different 
optimization solvers will be tested, and combined heat-and-flow experiments will be carried out in order to validate this technique experimentally. When nanosensors are determined to be suitable for field use, large-yield synthesis methods will be developed, and a tracer experiment will be performed in a geothermal field using these nanosensors. 



\section{Nomenclature}

$$
\begin{array}{ll}
t_{t h} & =\text { thermal breakthrough time } \\
t_{c} & =\text { chemical breakthrough time } \\
b & =\text { fracture aperture } \\
K_{r} & =\text { rock thermal conductivity } \\
\rho_{w} & =\text { water density } \\
\rho_{r} & =\text { rock density } \\
C_{w} & =\text { water heat capacity } \\
C_{r} & =\text { rock heat capacity } \\
C_{w} & =\text { water heat capacity } \\
k_{g a s} & =\text { gas permeability } \\
\mu & =\text { gas viscosity } \\
q & =\text { volumentric flowrate } \\
L & =\text { flowpath length } \\
A & =\text { flowpath cross-sectional area } \\
p_{i n} & =\text { inlet pressure } \\
p_{\text {out }} & =\text { outlet pressure } \\
\phi & =\text { porosity } \\
V_{p} & =\text { pore volume of sand-packed slim-tube } \\
V_{b} & =\text { bulk volume of sand-packed slim-tube } \\
V_{p 1} & =\text { pore volume of entire apparatus } \\
V_{p 2} & =\text { dead volume of inlet tubes } \\
W_{s} & =\text { weight of water column before saturation } \\
W_{d} & =\text { weight of water column after saturation } \\
r & =\text { radius of sand-packed tube } \\
l & =\text { length of sand-packed tube } \\
r_{t} & =\text { radius of inlet tubes } \\
l_{t} & =\text { length of inlet tubes } \\
k_{\text {liq }} & =\text { gas permeability } \\
\Delta p & =\text { pressure differential } \\
F_{m} & =\text { magnetic force } \\
\mu_{0} & =\text { permeability of free space (constant) }
\end{array}
$$




$$
\begin{aligned}
& V_{p} \quad=\text { nanoparticle volume } \\
& M_{p} \quad=\text { magnetization of nanoparticle } \\
& \nabla H=\text { magnetic field gradient } \\
& \eta_{\text {collection }}=\text { collection efficiency } \\
& m_{\text {collected }}=\text { mass of nanoparticles collected } \\
& m_{\text {injected }}=\text { mass of nanoparticles injected } \\
& C_{\text {collected }}=\text { concentration of nanoparticles in fluid collected } \\
& C_{\text {injected }}=\text { concentration of nanoparticles in fluid injected } \\
& V_{\text {collected }}=\text { volume of fluid collected } \\
& V_{\text {injected }}=\text { volume of fluid injected } \\
& C=\text { tracer concentration } \\
& x=\text { spatial coordinate } \\
& t=\text { time } \\
& u=\text { flux velocity } \\
& \alpha=\text { characteristic dispersion length } \\
& m=\text { tracer mass injected } \\
& q=\text { volumetric flowrate } \\
& V_{x}=\text { pore volume modified by tracer retardation factor } \\
& V_{\alpha} \quad=\text { dispersion volume modified by tracer retardation factor } \\
& T_{1}=\text { initial reservoir temperature } \\
& T_{2} \quad=\text { reservoir temperature after thermal drawdown } \\
& x_{f}=\text { position of thermal front } \\
& L \quad=\text { length of inter-well flowpath } \\
& \text { subscript }_{c}=\text { conservative tracer } \\
& \text { subscript }_{n}=\text { nanosensors } \\
& \text { subscript }_{r}=\text { released dye } \\
& x_{f, d}=\text { dimensionless position of thermal front }
\end{aligned}
$$




\section{References}

Alaskar, M., M. Ames, R. N. Horne, K. Li, S. Connor, and Cui Y. "In-situ Multifuction Nanosensors for Fractured Reservoir Characterization." Proc. of Geothermal Resources Council, Sacramento, CA.

Alaskar, Mohammed, Morgan Ames, Chong Liu, Steve Connor, Roland Horne, Kewen Li, and $\mathrm{Yi}$ Cui. "Smart Nanosensors for In-Situ Temperature Measurement in Fractured Geothermal Reservoirs." Proc. of Thirty-Sixth Workshop on Geothermal Reservoir Engineering, Stanford University, Stanford, CA.

Amyx, James W., Daniel M. Bass, and Robert L. Whiting. Petroleum Reservoir Engineering Physical Properties. New York: McGraw-Hill, 1960.

Behrens, H., I. Ghergut, M. Sauter, and T. Licha. "Tracer Properties, and Spiking Results (From Geothermal Reservoirs)." Proc. of Thirty-Fourth Workshop on Geothermal Reservoir Engineering, Stanford University, Stanford, CA. 2009.

Bertani, Ruggero. "Geothermal Power Generation in the World 2005-2010 Update Report." Proc. of World Geothermal Congress 2010, Bali, Indonesia.

Botterhuis, Nicole E., Qianyao Sun, Pieter C. M. M. Magusin, Rutger A. Van Santen, and Nico A. J. M. Sommerdijk. "Hollow Silica Spheres with an Ordered Pore Structure and Their Application in Controlled Release Studies." Chemistry - A European Journal 12.5 (2006): 1448-456.

Chen, H., Z. Li, Z. Wu, and Z. Zhang. "A Novel Route to Prepare and Characterize Sn-Bi Nanoparticles." Journal of Alloys and Compounds 394.1-2 (2005): 282-85.

Chrysikopoulos, C. V. "Artificial Tracers for Geothermal Reservoir Studies." Environmental Geology 22.1 (1993): 60-70.

Cumbie, D. H., and L. D. McKay. "Influence of Diameter on Particle Transport in a Fractured Shale Saprolite." Journal of Contaminant Hydrology 37 (1998): 13957.

Digital image. Phase Diagrams \& Computational Thermodynamics. The National Institute of Standards and Technology. 7 July 2010. $<$ http://www.metallurgy.nist.gov/phase/solder/bisn.html $>$.

Gentier, Sylvie, Xavier Rachez, Tien Dung Tran Ngoc, Mariane Peter-Borie, and Christine Souque. "3D Flow Modelling of the Medium-Term Circulation Test Performed in the Deep Geothermal Site of Soultz-sous-Forêts (France)." Proc. of World Geothermal Congress 2010, Bali, Indonesia.

Gerber, Richard, and Robert R. Birss. High Gradient Magnetic Separation. Chichester: Research Studies, 1983.

Hermann, W. “Quantifying Global Exergy Resources.” Energy 31.12 (2006): 1685702. 
Horne, R. N. "Geothermal Energy Worldwide Use - 2010." Stanford University. 29 Mar. 2011. Lecture.

Horne, R. N. "Reservoir Engineering of Reinjection." UN University, Geothermal Training Program, Reykjavik, Iceland. 2010. Lecture.

Juliusson, E., and Roland N. Horne. "Analyzing Tracer Tests During Variable Flow Rate Injection and Production." Proc. of Thirty-Sixth Workshop on Geothermal Reservoir Engineering, Stanford University, Stanford, CA.

Kanj, Mazen Y., Jim J. Funk, and Zuhair Al-Yousif. "Nanofluid Coreflood Experiments in the ARAB-D." Proc. of SPE Saudi Arabia Section Technical Symposium and Exhibition, Al-Khobar, Saudi Arabia. 2009.

"K\&J Magnetics - Magnet Calculator." K\&J Magnetics - Strong Neodymium Magnets, Rare Earth Magnets. 20 May 2011. $<$ http://www.kjmagnetics.com/calculator.asp $>$.

Kreft, A., and A. Zuber. "On The Physical Meaning Of the Dispersion Equation and Its Solutions For Different Initial and Boundary Conditions." Chemical Engineering Science 33 (1978): 1471-480.

Lund, John W., Derek H. Freeston, and Tonya L. Boyd. "Direct Utilization of Geothermal Energy 2010 Worldwide Review." Proc. of World Geothermal Congress 2010, Bali, Indonesia.

Moeser, Geoffrey D., Kaitlin A. Roach, William H. Green, T. Alan Hatton, and Paul E. Laibinis. "High-Gradient Magnetic Separation of Coated Magnetic Nanoparticles." AIChE Journal 50.11 (2004): 2835-848.

Nottebohm, Martin, Tobias Licha, Iulia Ghergut, Karsten Nödler, and Martin Sauter. "Development of Thermosensitive Tracers for Push-Pull Experiments in Geothermal Reservoir Characterization." Proc. of World Geothermal Congress 2010, Bali, Indonesia.

Plummer, Mitchell A., Carl D. Palmer, Earl D. Mattson, and Laurence C. Hull. "Sensitivity of a Reactive- Tracer Based Estimate of Thermal Breakthrough in an EGS to Properties of the Reservoir and Tracer." Proc. of Thirty-Sixth Workshop on Geothermal Reservoir Engineering, Stanford University, Stanford, CA. 2010.

Plummer, Mitchell A., Carl D. Palmer, Laurence C. Hull, and Earl D. Mattson. "A Reactive Tracer Analysis Method For Monitoring Thermal Drawdown In Geothermal Reservoirs." Proc. of Thirty-Fifth Workshop on Geothermal Reservoir Engineering, Stanford University, Stanford, CA. 2011.

Redden, George, Mark Stone, Karen E. Wright, Earl Mattson, Carl D. Palmer, Harry Rollins, Mason Harrup, and Laurence C. Hull. "Tracers For Characterizing Enhanced Geothermal Systems." Proc. of Thirty-Fifth Workshop on Geothermal Reservoir Engineering, Stanford University, Stanford, CA. 2010.

Reich, Martin, Satoshi Utsunomiya, Stephen E. Kesler, Lumin Wang, Rodney C. Ewing, and Udo Becker. "Thermal Behavior of Metal Nanoparticles in Geologic Materials." Geology 34.12 (2006): 1033. 
Reimus, Paul W. The Use of Synthetic Colloids in Tracer Transport Experiments in Saturated Rock Fractures. PhD Thesis. University of New Mexico, 1995.

Reimus, Paul W. "Transport and Attenuation of Carboxylate-Modified-Latex Microspheres In Fractured Rock Field Tracer Tests." Proc. of Interfacial and Colloidal Phenomena In Aquatic Environments, Anaheim, CA. 1999.

Robinson, Bruce A., and Stephen A. Birdsell. "Tracking Thermal Fronts With Temperature-Sensitive, Chemically Reactive Tracers." Fifth Geothermal Program Review, Washington, D.C. 1987.

Robinson, Bruce A., Jefferson W. Tester, and Lee F. Brown. "Using Chemically Reactive Tracers to Determine Temperature Characteristics of Geothermal Reservoirs." Proc. of Geothermal Resources Council, Reno, NV. 1984.

Rose, Peter, David Riasetto, Jacqueline Siy, Michael Bartl, Paul Reimus, Michael Mella, Kevin Leecaster, and Susan Petty. "Quantum Dots As Tracers In Geothermal and EGS Reservoirs." Proc. of Thirty-Sixth Workshop on Geothermal Reservoir Engineering, Stanford University, Stanford, CA. 2011.

Rose, Peter E., and Michael C. Adams. "The Application of Rhodamine WT As a Geothermal Tracer.” Proc. of Geothermal Resources Council. Vol. 18. 1994.

Saggaf, M.M. "A Vision for Future Upstream Technologies." Journal of Petroleum Technology 60.3 (2008).

Sankaran, A. V. "Recent Concepts about Heat Source from Earth's Core." Current Science 83.8 (2002): 932-34.

Shook, G. M. "Prediction of Thermal Breakthrough from Tracer Tests." Proc. of Twenty-Fourth Workshop on Geothermal Reservoir Engineering, Stanford University, Stanford, CA. 1999.

Sing, Charles E., Jill Kunzelman, and Christoph Weder. "Time-temperature Indicators for High Temperature Applications." Journal of Materials Chemistry 19.1 (2008): 104.

Sutton, Damon, Norased Nasongkla, Elvin Blanco, and Jinming Gao. "Functionalized Micellar Systems for Cancer Targeted Drug Delivery." Pharmaceutical Research 24.6 (2007): 1029-046.

Tester, J. W., B. A. Robinson, and J. H. Ferguson. "Inert and Reacting Tracers for Reservoir Sizing in Fractured, Hot Dry Rock Systems." Proc. of Eleventh Workshop on Geothermal Reservoir Engineering, Stanford University, Stanford, CA. 1986.

Tester, Jefferson W., Bruce A. Robinson, and James Ferguson. "The Theory and Selection of Chemically Recative Tracers For Reservoir Thermal Capacity Production." Proc. of Twelfth Workshop on Geothermal Reservoir Engineering, Stanford University, Stanford, CA. 1987.

Tester, Jefferson W., David D. Blackwell, Susan Petty, Michael C. Moore, Brian J. Anderson, Bill Livesay, Ron DiPippo, Kenneth Nichols, Ralph W. Veatch, Elisabeth M. Drake, M. Nafi Toksoz, Anthony S. Batchelor, John Garnish, Roy Baria, Chad Augustine, Enda Murphy, Petru Negraru, and Maria Richards. "The 
Future of Geothermal Energy: The Future of Impact of Enhanced Geothermal Systems (EGS) on the United States in the 21st Century." Tech. Massechusetts Institute of Technology, 2006.

U.S. Energy Information Administration. "EIA - 2010 International Energy Outlook Electricity." 20 May 2011. <http://www.eia.doe.gov/oiaf/ieo/electricity.html>.

Wu, Elizabeth C., Ji-Ho Park, Jennifer Park, Ester Segal, Frédérique Cunin, and Michael J. Sailor. "Oxidation-Triggered Release of Fluorescent Molecules or Drugs from Mesoporous Si Microparticles.” ACS Nano 2.11 (2008): 2401-409.

Yu, H., C. Kotsmar, K. Y. Yoon, D. R. Ingram, K. P. Johnston, S. L. Bryant, and C. Huh. "Transport and Retention of Aqueous Dispersions of Paramagnetic Nanoparticles in Reservoir Rocks." Proc. of 2010 SPE Improved Oil Recovery Symposium, Tulsa, OK. 2010. 
